\title{
On the Efficacy of State Space Reconstruction Methods in Determining Causality*
}

\author{
Bree Cummins ${ }^{\dagger}$, Tomáš Gedeon ${ }^{\dagger}$, and Kelly Spendlove
}

\begin{abstract}
We present a theoretical framework for inferring dynamical interactions between weakly or moderately coupled variables in systems where deterministic dynamics plays a dominating role. The variables in such a system can be arranged into an interaction graph, which is a set of nodes connected by directed edges wherever one variable directly drives another. In a system of ordinary differential equations, a variable $x$ directly drives $y$ if it appears nontrivially on the right-hand side of the equation for the derivative of $y$. Ideally, given time series measurements of the variables in a system, we would like to recover the interaction graph. We introduce a comprehensive theory showing that the transitive closure of the interaction graph is the best outcome that can be obtained from state space reconstructions in a purely deterministic system. Our work depends on extensions of Takens' theorem and the results of Sauer et al. [J. Stat. Phys., 65 (1991), pp. 579-616] that characterize the properties of time-delay reconstructions of invariant manifolds and attractors. Along with the theory, we discuss practical implementations of our results. One method for empirical recovery of the interaction graph is presented by Sugihara et al. [Science, 338 (2012), pp. 496-500], called convergent cross-mapping. We show that the continuity detection algorithm of Pecora et al. [Phys. Rev. E, 52 (1995), pp. 3420-3439] is a viable alternative to convergent cross-mapping that is more consistent with the underlying theory. We examine two examples of dynamical systems for which we can recover the transitive closure of the interaction graph using the continuity detection technique. The strongly connected components of the recovered graph represent distinct dynamical subsystems coupled through one-way driving relationships that may correspond to causal relationships in the underlying physical scenario.
\end{abstract}

Key words. state space reconstruction, delay embedding, Takens' theorem, chaotic attractor, partial order, lattice, filtration, causality, convergent cross-mapping

AMS subject classifications. $34 \mathrm{C} 45,34 \mathrm{D} 45,37 \mathrm{C} 20$

DOI. $10.1137 / 130946344$

1. Introduction. Over the past few decades, the development of high throughput technologies in the sciences has enabled us to collect massive amounts of data inexpensively and efficiently. We have terabytes of data on different complex processes from global warming, changing ocean currents, microbiome metagenomic data, to single-cell gene expression. The analysis of these data remains one of the great challenges. In particular, a topic of much interest is the identification of causal relationships between measured quantities to deduce their underlying network structure.

\footnotetext{
* Received by the editors November 25, 2013; accepted for publication (in revised form) by T. Sauer December 5, 2014; published electronically March 4, 2015. This research was supported by DARPA grant D12AP200025.

http://www.siam.org/journals/siads/14-1/94634.html

${ }^{\dagger}$ Mathematical Sciences, Montana State University, Bozeman, MT 59717 (cummins@math.montana.edu, gedeon@math.montana.edu). The work of the second author was partially supported by NSF grant DMS-1226213 and NIH R01 grant 1R01AG040020-01.

${ }^{\ddagger}$ Department of Mathematics, Rutgers University, Hill Center - Busch Campus, Piscataway, NJ 08854 (kelly. spendlove@rutgers.edu).
} 
It has been long recognized that correlation between two variables does not imply causation. Correlated variables can both be driven by a third hidden variable. At the same time, in nonlinear dynamical systems, two variables that drive each other's evolution in time can be correlated at some times, anticorrelated at others, and have no correlation at yet other times [19].

The framework of Granger causality (GC) [7] uses predictability to identify causation between two time series. A variable $x$ is said to "Granger cause" $y$ if the predictability of $y$ declines when $x$ is removed from the set of all possible causative variables [7]. The key assumption for applicability of GC is separability; that is, the information about $x$ can be removed from the system by eliminating that variable from the model. Separability is characteristic of purely stochastic and linear systems, but not present in complex interconnected systems where many variables are mutually coupled. In such systems information about one variable $x$ is contained in the variable $y$ and cannot be formally removed without severely altering the dynamics of other variables.

Recently, Sugihara et al. [19] proposed a method to detect causal relationships based on the state space reconstruction of time series data. This approach, dubbed the convergent crossmapping technique (CCM), is complementary to the GC approach. While GC is designed for stochastic processes, CCM applies to weakly or moderately coupled variables in systems where deterministic dynamics plays a dominating role. At the root of CCM is Takens' embedding theorem, which states that for an invariant manifold $M$, generic observation function $x(t)$, and delay $T$ (explained in greater detail in section 2.1) a delay reconstruction of $M$ is diffeomorphic to $M$ itself. If one assumes that the coordinate times series $x(t)$ and $y(t)$ belong to the generic set, then the reconstructions $M_{x}$ using $x(t)$ and $M_{y}$ using $y(t)$ are diffeomorphic via their diffeomorphism to $M$. Therefore one can estimate elements of $M_{y}$ using information from $M_{x}$, and vice versa. Sugihara et al. [19] assert that if one can predict states in $M_{y}$ based on information in $M_{x}$, then $x$ is directionally causally related to $y$ in the sense that $x$ drives $y$. The assessment of predictability is based on both the quality of the prediction and the detection of improvement of the quality as a function of the length of the time series.

In this contribution we will develop a precise mathematical theory that builds on Takens' theorem and puts CCM on a solid mathematical framework. This allows us to illuminate the strengths and limitations of CCM in both theory and practice.

Our starting point is a dynamical system with $n$ variables, described by a deterministic map or a set of differential equations. We will develop parallel theories for both a system evolving on an invariant manifold, where we use the embedding framework of Takens [20], or a system on a global attractor, where we use the results of Sauer, Yorke, and Casdagli [16]. The structure of the dynamical system determines an interaction graph between variables; if a variable $x$ occurs on the right-hand side of the evolution of variable $y$, then $x$ is a dynamical driver of $y$. We assume that we have access to time series of all $n$ variables, perhaps corrupted by a measurement noise, and our goal is to recover the structure of the dynamical drivers in the system, i.e., the interaction graph.

In the first part of the paper, we make basic definitions and mathematical structures that are similar for both the manifold and attractor approaches. Then we focus on extending Taken's invariant manifold approach, and finally we modify our results from the manifold viewpoint to the attractor viewpoint, outlining our work in a very similar fashion.

Copyright (c) by SIAM. Unauthorized reproduction of this article is prohibited. 
If two variables $x$ and $y$ are in the same connected component of the interaction graph, then provided that the projections of the dynamical system onto $x$ and $y$ belong to a generic set of observation functions, their reconstructions will be diffeomorphic, a result that follows immediately from Takens' Theorems 3.1 and 3.3. This motivates the definition of a coarser component graph whose vertices are connected components of the interaction graph. We show that the set of all potentially different reconstructions is parameterized by a lattice of upper sets of the component graph, which is introduced in section 2.3 and referenced frequently in the remainder of the paper. The parameterization occurs because an upper set of a set of variables includes all potential dynamical drivers of these variables.

We introduce the concept of fully resolved manifold structure where there is a different (i.e., nonhomeomorphic) manifold for each upper set in Definition 3.8. Only fully resolved manifold structures have sufficient richness of dynamics to allow the reconstruction of the full component graph. This is related to the observation of Sugihara et al. [19] that if a driver variable $z$ drives two other variables $x$ and $y$ so strongly that they become synchronized, CCM will not be able to distinguish this from mutual dynamical driving between $x$ and $y$. In our view this is a sign that the manifolds corresponding to the upper sets $\{Z, X\}$ and $\{Z, Y\}$ (explained in more detail in section 2.3) are homeomorphic, and thus the manifold structure is not fully resolved.

We also show in Theorem 3.11(b) that for any two different manifolds $M_{U}, M_{V}$ that are parameterized by two upper sets with $U \subset V$ there is a projection $M_{V} \rightarrow M_{U}$. This corresponds to the concept of "unidirectional causality" in the CCM method.

We show that the lattice of upper sets induces filtrations of the global invariant manifold in section 2.4. By extending the embedding theorem of Takens [20], we define a generic set of observation functions with the same lattice structure (section 3.2). If the single coordinate observation functions are in this generic set, one can recover the component graph of a fully resolved system almost completely. The recovery is based on creating the reconstructions $M_{x}$ for each time series $x$, and then detecting which reconstructions are homeomorphic to each other (this detects mutual driving), which ones are projections of each other (detects one-directional driving), and which are unrelated (Algorithm 1). We show that this approach cannot recover self-loops, and, further, one cannot distinguish between mutual versus one-directional dynamical driving within connected components of the interaction graph in Theorem 3.13. We follow similar steps for the attractor framework in section 4, building on the theorems of Sauer, Yorke, and Casdagli [16].

Finally, we suggest an alternative approach to the CCM algorithm in section 5. While $\mathrm{CCM}$ concentrates on the predictability of a time series $y(t)$ based on the information from the reconstructed manifold $M_{x}$, we implement an algorithm by Pecora, Carroll, and Heagy[15], which tests the hypothesis that two reconstructions are continuous images of each other. In agreement with the CCM approach, we detect continuity based on both the quality of the fit and the convergence trends with the length of the time series. While our approach is more consistent with the underlying theory, it is closely related to CCM.

We do not claim to recover causal relationships using this method, because the assignment of causality involves the interpretation of the variables in a dynamical system. For example, suppose there are two pendulums, $p_{1}$ and $p_{2}$, that are perfectly synchronized, perhaps by a hidden and unmeasured mechanism. The second one, $p_{2}$, has an additional chaotic pendulum

Copyright (c) by SIAM. Unauthorized reproduction of this article is prohibited. 
hanging from its bob. The independent pendulum $p_{1}$ obviously is not the cause of the chaotic pendulum's motion, but because it is synchronized to the proximal cause of motion, $p_{2}$, it makes no difference whatsoever which of $p_{1}$ or $p_{2}$ are measured. In this case, the measured motion of $p_{1}$ is a proxy variable for $p_{2}$; it is a (perfect) correlate of $p_{2}$. In the terminology of this paper, both $p_{1}$ and $p_{2}$ are dynamical drivers of the chaotic pendulum. Our methods recover the direct and indirect dynamical drivers of a variable within a given set of measurements. These relationships form a set of hypothetical causes that must be assessed in light of their physical interpretations before causality is inferred.

The paper is organized in the following way. In section 2 we formalize the interaction and component graphs, lattices, and filtrations mentioned above. In section 3 we extend Takens' theorem regarding embedding invariant manifolds to a filtration structure. We then summarize the algorithm for recovering the interaction and component graphs of a set of time series. In section 4 we use the theorems of Sauer, Yorke, and Casdagli [16] to show that the same results apply to the attractors of the system. In section 5, we describe the application of the method of Pecora, Carroll, and Heagy [15] to the recovery of the component graph of a system and present several examples. We conclude with a brief summary and discussion.

\section{Decomposition of invariant manifolds and attractors.}

2.1. Preliminaries. A dynamical system is a collection of maps $\phi^{t}: \mathbb{R}^{n} \rightarrow \mathbb{R}^{n}$, parameterized by either $t \in \mathbb{Z}, t \in \mathbb{Z}^{+}$, or $t \in \mathbb{R}$. In the first two cases the dynamical system may be generated by iterates of either a diffeomorphism or a smooth map $\phi$. In the last case it may be generated by solutions of a system of differential equations.

Restricting $\phi$ to some compact, invariant, smooth manifold $M$ of dimension $m$, the celebrated theorem of Takens shows that under very general conditions on a dynamical system $(M, \phi)$, for a generic smooth observation function $\varphi: M \rightarrow \mathbb{R}$ and a generic choice of $T>0$, the following delay reconstruction map is an embedding of $M$ into $\mathbb{R}^{2 m+1}$ :

$$
\Phi_{\left(\phi^{T}, \varphi, 2 m\right)}: M \rightarrow \mathbb{R}^{2 m+1}:=\left(\varphi(x), \varphi\left(\phi^{-T}(x)\right), \varphi\left(\phi^{-2 T}(x)\right), \ldots, \varphi\left(\phi^{-2 m T}(x)\right)\right) .
$$

Note that the components correspond to time-lagged observations of the dynamics on $M$ moderated by scalar observation function $\varphi$. For this paper, any $C^{k}$ scalar function $\varphi: M \rightarrow \mathbb{R}$ will be referred to as an observation function. As will be pointed out later, for Takens' framework $k=2$ is needed, but in the Sauer, Yorke, Casdagli [16] approach $k=1$ is sufficient.

The following discussion will be carried out for a general dynamical system on a compact $m$-dimensional invariant smooth manifold $M$ that is a submanifold of Euclidean space $\mathbb{R}^{n}$. While the observation function in Takens' theorem can be arbitrary, it is often the case that the observation function is a projection onto a single variable, or, in other words, a coordinate in $\mathbb{R}^{n}$. Takens' theorem states one may be able to reconstruct the invariant manifold from observations of a single variable of the system (see the Lotka-Volterra example below). If there exists such an observation function obtained from $x$, we say variable $x$ reconstructs $M$.

Of course there are exceptions, and some variables cannot reconstruct the original manifold. One of the reasons for this may be symmetry in the manifold which causes a particular variable $w$ to lie in a fixed subspace of a symmetry transformation. Then the variables related by symmetry, but not fixed by the symmetry, cannot be distinguished based on the observation of $w$. 
Another reason a variable may not reconstruct $M$, which is the main focus of this paper, is an interaction structure of the set of variables. If the dynamics of the observed variable $w$ is unaffected by another variable $x$, then the reconstruction from $w$ cannot reconstruct dynamics of variable $x$. Sugihara et al. [19] have recently introduced a method based on Takens' theorem which attempts to infer the interaction relationship between two variables.

Often one may be more interested in embedding an attractor of a system, rather than some invariant manifold it lies on. In many cases, the attractor is not a compact submanifold of the phase space (indeed, they can be notoriously complex objects), and thus one cannot invoke Takens' theorem. In Sauer, Yorke, and Casdagli [16], results were established showing that if $m$ is larger than twice the box-counting dimension of attractor $A$, then for $U$ open with $A \subset U$, "almost every" map from $U \rightarrow \mathbb{R}^{m}$ is one-to-one on $A$ and an immersion on compact subsets of smooth manifolds contained within $A$. Both Takens' theorem and the work of Sauer, Yorke, and Casdagli [16] will play a key role in this work.

2.2. Interaction graph. We will now introduce the notion of an interaction graph, which is a directed graph reflecting the dynamical interaction between variables in the dynamical system.

Definition 2.1. Given a dynamical system $\phi$, and two variables $x$ and $y$ of $\phi$, the variable $x$ directly drives $y$ if the dynamics of $y$ directly depend on $x$. In the case of a diffeomorphism, or a set of differential equations, this means that the right-hand side of the evolution equation for $y$ has nontrivial dependence on $x$, i.e., $y_{t+1}=g(x, \cdot)$ or $\dot{y}=g(x, \cdot)$. We exclude trivial dependence where changes in $x$ do not cause any changes in $g(x, \cdot)$.

Definition 2.2. Given a dynamical system $\phi$, the interaction graph is a directed graph $\mathcal{I G}_{\phi}=\left(V_{\phi}, E_{\phi}\right)$ with the set of vertices $V_{\phi}$ corresponding to variables of $\phi$ and a directed edge from vertex $x$ to $y$ if $x$ directly drives $y$. To simplify the notation, we often drop the subscript $\phi$ when the dynamical system under consideration is clear from the context.

As an example, consider the Lotka-Volterra model, part of a broad class of predator-prey systems, which is a pair of first order, nonlinear, differential equations used to describe the dynamics of a biological systems in which two species interact, with one being predatory and the other its prey. The equations are given by

$$
\begin{aligned}
& \frac{d u}{d t}=a u-b u v, \\
& \frac{d v}{d t}=-c v+d b u v .
\end{aligned}
$$

Here $u$ is the prey, $v$ is the predator, and $a, b, c, d$ are parameters.

For some parameter sets, the above system exhibits a periodic orbit, which is depicted in Figure 1, along with both corresponding time series.

Notice that both variables in the system are involved in each other's production: $u$ directly drives $v$, and vice versa. The resulting interaction graph is shown in Figure 2.

Further, since the system does not show any obvious symmetries, observations of either $u$ or $v$ should yield generic observation functions. Thus Takens' theorem allows one to reconstruct the invariant 1-manifold (periodic orbit) from delay reconstruction of either time series. Figure 3 shows the two-dimensional reconstructions from each variable. 


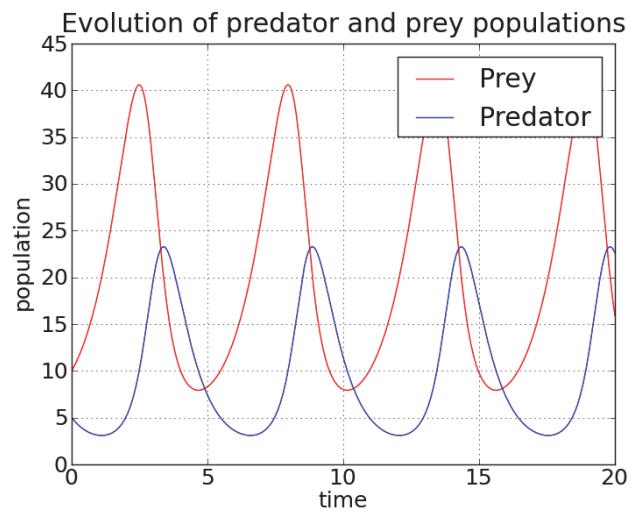

(a)

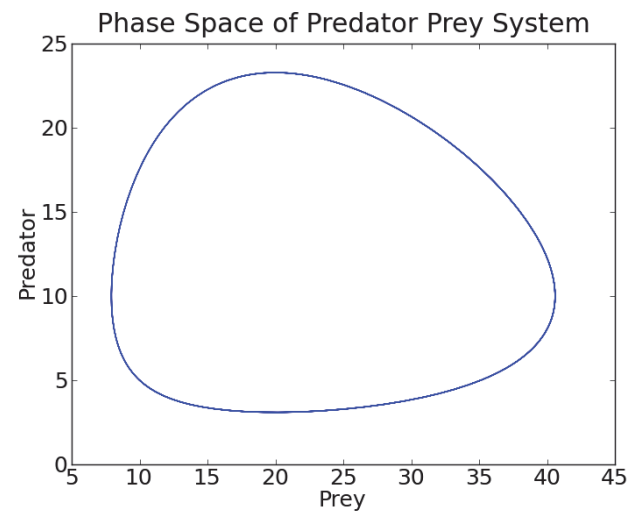

(b)

Figure 1. Lotka-Volterra model: (a) time series; (b) periodic orbit in phase space.

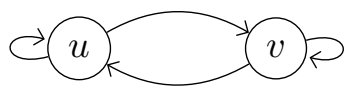

Figure 2. Interaction graph for the Lotka-Volterra system.

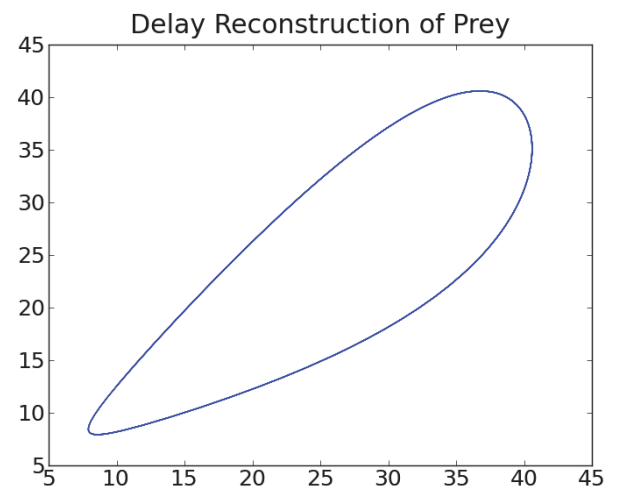

(a)

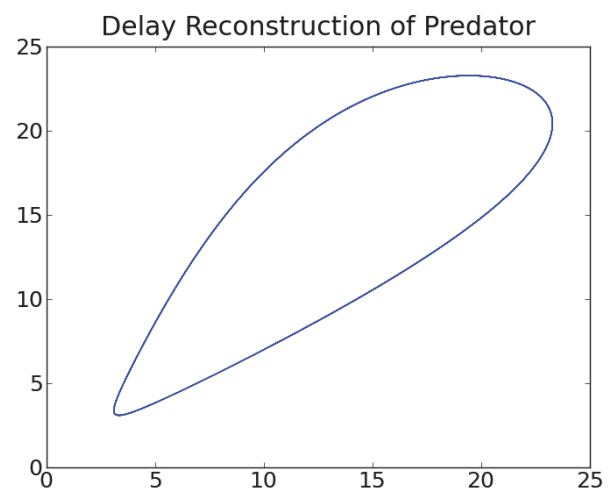

(b)

Figure 3. Delay reconstructions from (a) u variable (prey); (b) v variable (predator).

2.3. Component graph. We want to show that the interaction structure between variables in the dynamical system naturally leads to a hierarchy of invariant manifolds or attractors that reflects the driving relationships between these variables. To develop the notion of such a hierarchy, we first coarsen the idea of interaction graphs. This requires some notions from graph theory.

Definition 2.3. For directed graph $G=(V, E)$ and $u, v \in V, u$ is reachable from $v$ if there exists a directed path of edges in $E$ from $v$ to $u$. In particular, every vertex is reachable from itself via the trivial path.

Copyright ( by SIAM. Unauthorized reproduction of this article is prohibited. 
Notice that $v$ may still be reachable from $u$ even if $u$ does not directly drive $v$. In this case the dynamics of variable $u$ indirectly influence the production of $v$. We take the sets of mutually reachable vertices to be the basic unit of interaction using the notion of a strongly connected component.

Definition 2.4. A subset of vertices $W \subset V$ is a strongly connected component if it is a maximal subset such that for any $u, v \in W, u$ is reachable from $v$ and $v$ is reachable from $u$.

Note that belonging to the same strongly connected component is an equivalence relation. Thus the set of strongly connected components partitions the graph. We denote the set of strongly connected components by $\mathcal{S}$. Although two variables in the same strongly connected component may not directly drive one another, they are still governed in part by each other's dynamics due to the strongly connected component structure. This motivates the following definition.

Definition 2.5. Given a dynamical system $\phi$ and its interaction graph $\mathcal{I} \mathcal{G}_{\phi}$, the component graph of $\phi$ is obtained by taking each strongly connected component $q \in \mathcal{S}$ to be a vertex and declaring an edge from $q \rightarrow p$ if, for some (and therefore any) $v \in q$ and $u \in p, u$ is reachable from $v$. We denote this graph by $\mathrm{CG}_{\phi}=\left(\mathcal{S}_{\phi}, U_{\phi}\right)$.

Notice that any component graph must be an acyclic directed graph and can therefore be thought of as a partially ordered set (poset) $(\mathcal{S}, \leq)$. We will refer to the component graph as both a graph and poset, the partial order on $\mathcal{S}$ being induced from the directed graph, i.e., $q \geq p$ if there exists an edge $q \rightarrow p$ in the transitive closure of $\mathrm{CG}_{\phi}$. This order $\mathcal{S}$ captures a coarse interaction structure since if $q \geq p$, then there is a path along directed edges in $\mathbf{I G}_{\phi}$ from at least one variable in $q$ to at least one variable in $p$. If we are interested in all indirect drivers to a given variable, we are lead to the notion of an upper set in the partial order CG: an upper set $U$ is a subset $U \subset \mathcal{S}$ with the property that if $p \in U$ and $q \geq p$, then $q \in U$.

As we will show, the significance of an upper set $U$ of CG is that for a dynamical system $\phi$ with an interaction graph $\mathcal{I} \mathcal{G}$ there is an associated well-defined dynamical system $\phi_{U}$ obtained by restricting dynamics to the variables in the upper set. To describe the relationship between the dynamical systems $\phi_{U}$ corresponding to upper sets $U \subset C G$ we introduce the notion of lattice of upper sets. Note that the upper sets of CG form a distributive lattice in which the partial ordering is given by set inclusion: the join corresponds to union and the meet to intersection; the highest element of this lattice represents all vertices of the component graph CG and hence all variables of the system. We denote this lattice $U L_{\phi}$. An example can be seen in Figure 4.

The component graph and associated lattice of upper sets encode the driving relationships between the variables of the system. These structures allow us to decompose the invariant manifold or attractor of the system in a natural fashion. We begin with a definition of a structure for decomposing the manifold, for which $U L_{\phi}$ will serve as an indexing set. We adapt slightly the notion of a filtration, which usually requires that the indexing set is well ordered; in our case $U L_{\phi}$ is only partially ordered.

\subsection{Filtration of invariant manifolds and attractors.}

Definition 2.6. A filtration $\mathbb{F}$ is a collection of subsets $X_{a}$ of a set $X$ indexed by a poset $I$, together with a collection of inclusion maps $\iota_{a b}: X_{a} \hookrightarrow X_{b}$, for every pair of indices satisfying $a \leq b$ in the partial order. This collection is consistent; that is, for all ordered triples $a \leq b \leq c$ 

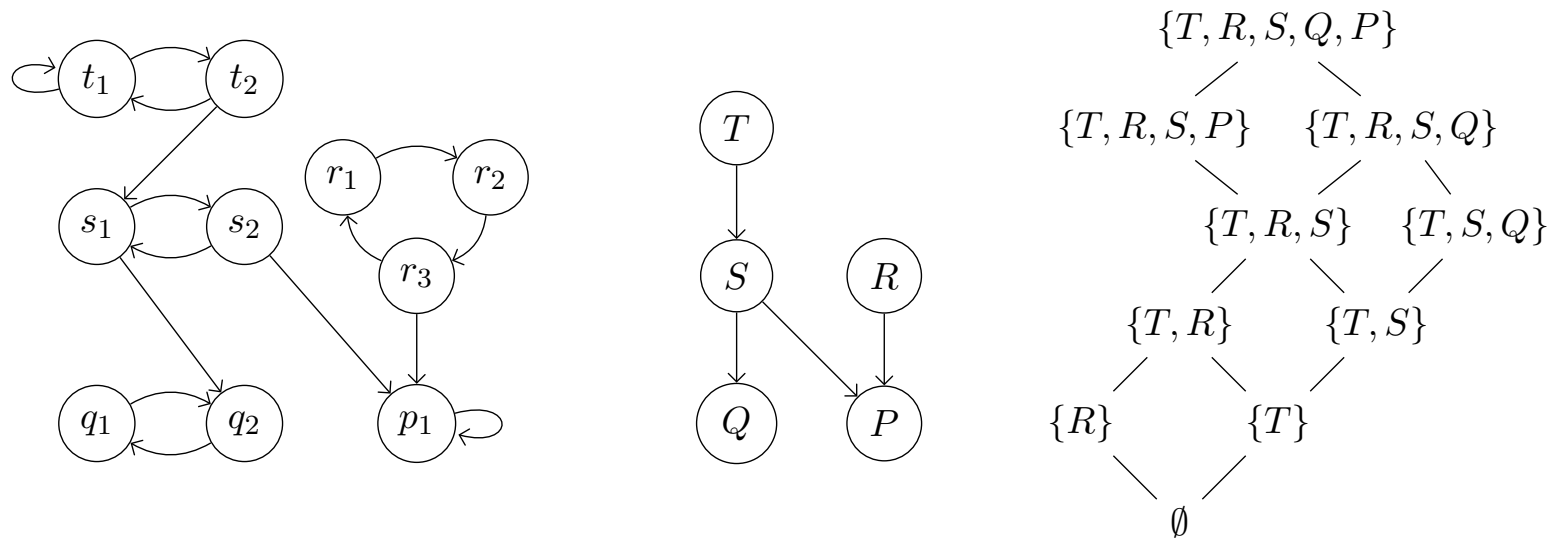

Figure 4. Example $\mathcal{I G}_{\phi}, \mathrm{CG}_{\phi}$, and associated lattice of upper sets $U L_{\phi}$. We will use this example throughout this paper.

we have

$$
\iota_{b c} \circ \iota_{a b}=\iota_{a c}
$$

$A$ descending filtration is a collection of subsets indexed by a poset, together with a collection of projection maps $\pi_{b a}: X_{b} \rightarrow X_{a}$, for every pair of indices satisfying $a \leq b$ in the partial order. Furthermore, this collection is consistent; that is, for all ordered triples $a \leq b \leq c$ we have

$$
\pi_{b a} \circ \pi_{c b}=\pi_{c a}
$$

Given the lattice $U L_{\phi}$ as an index set we define a descending filtration of the phase space $\mathbb{R}^{n}$. To each element $U \in U L_{\phi}$ we define $|U|$ to be the set of variables corresponding to vertices in $\mathcal{I G}$ that belong to $U$. Let $\mathbb{R}_{U} \subset \mathbb{R}^{n}$ be a subspace spanned by the variables in $|U|$. If $n_{U}$ is the number of variables in $U$, then $\mathbb{R}_{U}$ has dimension $n_{U}$ (in other words, $\mathbb{R}_{U}$ is isomorphic to $\left.\mathbb{R}^{n_{U}}\right)$. Notice that the partial order on $U L_{\phi}$ implies that

$$
\mathbb{R}_{U} \subset \mathbb{R}_{V} \quad \text { if and only if } \quad U<V \quad \text { if and only if } \quad|U| \subset|V| \text {. }
$$

In particular, there is a natural projection

$$
\pi_{V U}: \mathbb{R}_{V} \rightarrow \mathbb{R}_{U}
$$

To describe the projection we can reorder variables in $\mathbb{R}_{U}$ and $\mathbb{R}_{V}$ in such a way that $\left\{x_{1}, \ldots, x_{n_{U}}\right\}$ are variables in $\mathbb{R}_{U}$ and $\left\{x_{1}, \ldots, x_{n_{U}}, x_{n_{U}+1}, \ldots, x_{n_{V}}\right\}$ are variables in $\mathbb{R}_{V}$. Then

$$
\pi_{V U}\left(x_{1}, \ldots, x_{n_{U}}, x_{n_{U}+1}, \ldots, x_{n_{V}}\right)=\left(x_{1}, \ldots, x_{n_{U}}\right) \text {. }
$$

It is easy to see that the collections of maps $\left\{\pi_{V U}\right\}_{U, V \in U L_{\phi}}$ satisfy the consistency condition (4). Notice that the supremum of $U L_{\phi}$ corresponds to collection of all variables. Therefore $\mathbb{R}_{\text {sup }}$ is the entire phase space $\mathbb{R}^{n}$, and we use the shorthand notation $\pi_{U}: \mathbb{R}^{n} \rightarrow \mathbb{R}_{U}$ to denote

Copyright (C) by SIAM. Unauthorized reproduction of this article is prohibited. 
the projection from $\mathbb{R}^{n}$ to $\mathbb{R}_{U}$. The subspaces $\mathbb{R}_{U}$ indexed by elements of the lattice $U L_{\phi}$, with natural projections discussed above, form a descending filtration that we will call the upper set filtration.

Proposition 2.7. Given a dynamical system $\phi$, for every pair $U, V \in U L_{\phi}$ with $U \leq V$ the projections $\pi_{U}$ and $\pi_{V U}$ commute with the dynamical system $\phi$. Therefore there is a well defined collection of dynamical systems $\left\{\phi_{W}^{t}\right\}_{W \in U L_{\phi}}$, with $\phi_{U}^{t}$ defined on $\mathbb{R}_{U}$ given by

$$
\phi_{U}^{t} \circ \pi_{U}(x)=\pi_{U} \circ \phi^{t}(x) \text { for all } x \in \mathbb{R}^{n} .
$$

Furthermore, the collection of dynamical systems $\left\{\phi_{W}^{t}\right\}_{W \in U L_{\phi}}$ is consistent; i.e., it satisfies

$$
\pi_{V U} \circ \phi_{V}^{t}(x)=\phi_{U}^{t} \circ \pi_{V U}(x) \text { for all } x \in \mathbb{R}_{V} .
$$

Proof. We need to show that $\phi_{U}^{t}$ is well defined. Assume that $\pi_{U}(x)=\pi_{U}(y)$ for some $x \neq y$. This means that $x$ and $y$ agree in all variables that correspond to vertices in $|U| \subset\left|V_{\phi}\right|$, where $\left|V_{\phi}\right|$ is the set of all vertices in the interaction graph $\mathcal{I} \mathcal{G}_{\phi}$, and can differ only in the variables that correspond to vertices $\left|V_{\phi}\right| \backslash|U|$. The key observation is that the equations that govern variables in $|U|$ do not depend on variables in $\left|V_{\phi}\right| \backslash|U|$. Therefore even though $\phi^{t}(x)$ differs from $\phi^{t}(y)$, they agree on all variables in $|U|$. Thus

$$
\pi_{U} \circ \phi^{t}(x)=\pi_{U} \circ \phi^{t}(y)
$$

and $\phi_{U}^{t}$ is well defined.

To show consistency, we use the consistency of the projection maps $\left\{\pi_{V U}\right\}_{U, V \in U L_{\phi}}$. In particular, if $U \leq V$, we have $\pi_{U}=\pi_{V U} \circ \pi_{V}$. Using this and (5), we get

$$
\pi_{U} \circ \phi^{t}(x)=\phi_{U}^{t} \circ \pi_{U}(x)=\phi_{U}^{t} \circ \pi_{V U} \circ \pi_{V}(x) .
$$

On the other hand

$$
\pi_{U} \circ \phi^{t}(x)=\pi_{V U} \circ \pi_{V} \circ \phi^{t}(x)=\pi_{V U} \circ \phi_{V}^{t} \circ \pi_{V}(x) .
$$

Comparing the two expressions, we get the consistency statement above.

Now assume that there is a global attractor $A$ in $\mathbb{R}^{n}$ under $\phi$, or, alternatively, that there is an invariant manifold $M$ for $\phi$ in $\mathbb{R}^{n}$. The next results show that these objects inherit the structure of the upper set filtration and are direct consequences of Proposition 2.7.

Corollary 2.8. Consider $\phi$ restricted to some compact invariant manifold $M$. There is a descending filtration $\mathbb{M}$ with index set $U L_{\phi}$, consisting of the collection $\left\{M_{U}\right\}_{U \in U L_{\phi}}$, where $M_{U}:=\pi_{U}(M)$. Further, each $M_{U}$ is a compact invariant set under the dynamical system $\phi_{U}^{t}$, defined in Proposition 2.7.

Proof. The continuous image of a compact set is compact, so each $M_{U}=\pi_{U}(M)$ is compact. Now let $y \in M_{U}$ and take any $z \in M$ such that $\pi_{U}(z)=y$. Then $\phi_{U}^{t}(y)=$ $\pi_{U} \circ \phi^{t}(z) \in \pi_{U}(M)=M_{U}$. Thus $M_{U}$ is a projection of $M$ that is a compact, invariant set under $\phi_{U}^{t}$.

Corollary 2.9. Consider $\phi$ restricted to some compact attractor $A$. There is a descending filtration $\mathbb{A}$ of the attractor $A$ with index set $U L_{\phi}$, consisting of the collection $\left\{A_{U}\right\}_{U \in U L_{\phi}}$, 
where $A_{U}:=\pi_{U}(A)$. Further, each $A_{U}$ is a compact attractor of the associated dynamical system $\phi_{U}^{t}$, defined in Proposition 2.7.

Proof. The argument for $A_{U}$ a compact, invariant set is identical to that for $M_{U}$ a compact, invariant set in Corollary 2.8. We also need to show that $A_{U}$ is an attractor of $\phi_{U}^{t}$. We use the definition of attractor given in Strogatz [18]: An attractor is a closed, invariant set that attracts an open set of initial conditions; further, it contains no proper subset with the same properties (i.e., it is minimal).

Assuming the subspace topology on $A \subset \mathbb{R}^{n}$, the projection $\pi_{U}$ is an open map. Therefore, an open set $O \supset A$ of initial conditions that are attracted to $A$ maps onto an open set $P:=\pi_{U}(O) \supset \pi_{U}(A)=A_{U}$ under the projection. Let $y \in P$, and take any $z \in O$ such that $\pi_{U}(z)=y$. Then by the definition, $\phi^{t}(z) \rightarrow A$ as $t \rightarrow \infty$. In particular, given $\epsilon>0$ there exists $T(\epsilon)$ such that $t>T(\epsilon)$ implies $\operatorname{dist}\left(\phi^{t}(z), A\right)<\epsilon$, where $\operatorname{dist}\left(\phi^{t}(z), A\right)=\inf \left\{\left\|\phi^{t}(z)-a\right\|\right.$, $a \in A\}$ and $\|\cdot\|$ is the norm on $\mathbb{R}^{n}$. Then $\operatorname{dist}\left(\phi_{U}^{t}(y), A_{U}\right)=\operatorname{dist}\left(\pi_{U}\left(\phi^{t}(z)\right), \pi_{U}(A)\right) \leq$ $\operatorname{dist}\left(\phi^{t}(z), A\right)<\epsilon$ when $t>T(\epsilon)$. So $\phi_{U}^{t}(y) \rightarrow A_{U}$ as $t \rightarrow \infty$ and the open set $P \supset A_{U}$ is attracting for $\phi_{U}^{t}$.

Suppose by contradiction that $A_{U}$ is not minimal; i.e., there exists closed $B_{U} \subsetneq A_{U}$ that is invariant under $\phi_{U}^{t}$ and there exists open $P \supset B_{U}$ of initial conditions that are attracted to $B_{U}$. By continuity, the preimages $\pi_{U}^{-1}\left(B_{U}\right)$ and $\pi_{U}^{-1}(P)$ are closed and open, respectively, in $\mathbb{R}^{n}$, so that $B:=\pi_{U}^{-1}\left(B_{U}\right) \cap A$ and $Q:=\pi_{U}^{-1}(P) \cap O$ are closed and open, respectively. Let $y \in B_{U}$ and $z \in B$ such that $\pi_{U}(z)=y$. Then $\phi^{t}(z) \in B$ for all time $t$, since otherwise the projection $\pi_{U}\left(\phi^{t}(z)\right)=\phi_{U}^{t}(y)$ would not be in $B_{U}$, a contradiction. So $B$ is a proper subset of $A$, because $B_{U} \subsetneq A_{U}$, which is also closed and invariant. Now let $y \in P \cap \pi_{U}(O)$, so that $\phi_{U}^{t}(y) \rightarrow B_{U}$ as $t \rightarrow \infty$. There must be $z \in Q$ such that $\pi_{U}(z)=y$. Then $\phi_{U}^{t} \circ \pi_{U}(z) \rightarrow B_{U}$ implies $\pi_{U} \circ \phi^{t}(z) \rightarrow B_{U}$ by (5), so that $\phi^{t}(z) \rightarrow \pi_{U}^{-1}\left(B_{U}\right)$. Since $z \in Q \subset O, \phi^{t}(z) \rightarrow$ $A$ as $t \rightarrow \infty$, so that $\phi^{t}(z) \rightarrow \pi_{U}^{-1}\left(B_{U}\right) \cap A \equiv B$. This shows that $Q$ is an open set of initial conditions that is attracted to $B$. This contradicts $A$ minimal; therefore $A_{U}$ must be minimal.

We call $\mathbb{A}$ an attractor filtration and $\mathbb{M}$ an invariant set filtration of the dynamical system $\phi$. The projection $\pi_{V U}: \mathbb{R}_{V} \rightarrow \mathbb{R}_{U}$ acting on real spaces is noninjective by construction for $U<V$, but the same projection acting on invariant sets or attractors, $\pi_{V U}: M_{V} \rightarrow M_{U}$ and $\pi_{V U}: A_{V} \rightarrow A_{U}$, could be injective. This can occur, for example, if two manifolds on different levels of the filtration are homeomorphic even though the associated dynamical systems $\phi_{U}^{t}$ and $\phi_{V}^{t}$ are in different dimensions. The presence or absence of injectivity in the projections controls how much of the dynamical structure of $\phi$ can be recovered, and later we will discuss constraining the filtrations $\mathbb{M}$ and $\mathbb{A}$ to noninjective projections for optimal information recovery.

2.5. Join irreducibility. In the example in Figure 5, consider the attractors $A_{\{T R S\}}$, $A_{\{T R\}}$, and $A_{\{T S\}}$ corresponding to the upper sets $\{T, R, S\},\{T, R\}$, and $\{T, S\}$, respectively. Since the upper set $\{T, R, S\}$ contains no variables other than those found in the sets $\{T, R\}$ and $\{T, S\}$, the attractor $A_{\{T R S\}}$ is completely determined by the attractors $A_{\{T R\}}$ and $A_{\{T S\}}$. Indeed, let

$$
\pi_{1}: A_{\{T R S\}} \rightarrow A_{\{T R\}} \text { and } \pi_{2}: A_{\{T R S\}} \rightarrow A_{\{T S\}}
$$

Copyright (c) by SIAM. Unauthorized reproduction of this article is prohibited. 

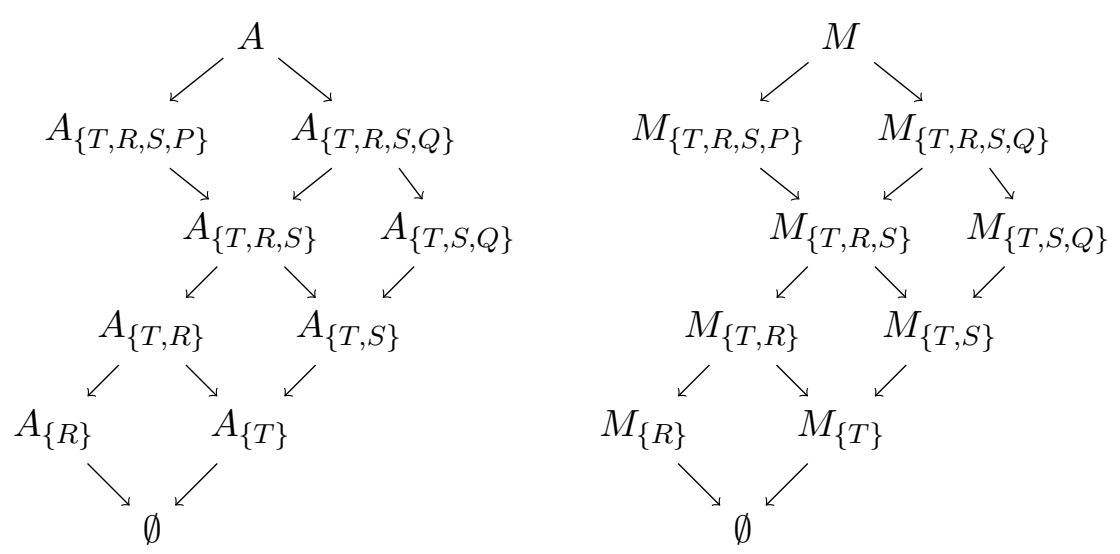

Figure 5. Descending filtrations $\mathbb{A}$ and $\mathbb{M}$ associated with $U L_{\phi}$ of Figure 4.

be the projections that correspond to set inclusions $\{T, R\} \hookrightarrow\{T, R, S\}$ and $\{T, S\} \hookrightarrow$ $\{T, R, S\}$, respectively. We want to express the observation that any trajectory $\varphi(x, t)$ in $A_{\{T R S\}}$ can be fully described by trajectories $\pi_{1}(\varphi(x, t))$ in $A_{\{T R\}}$ and $\pi_{2}(\varphi(x, t))$ in $A_{\{T S\}}$. Note that the trajectory $\varphi(x, t)$ is described by the set of coordinate scalar-valued trajectories $x_{j}(t)$ for all $x_{j} \in|T| \cup|R| \cup|S|$. If we denote the list of these trajectories by

$$
\{\varphi(x, t)\}:=\left\{x_{j_{1}}(t), \ldots, x_{j_{s}}(t)\left|x_{j_{i}} \in\right| T|\cup| R|\cup| S \mid\right\},
$$

then we can write

$$
\{\varphi(x, t)\}=\left\{\pi_{1}(\varphi(x, t))\right\} \cup\left\{\pi_{2}(\varphi(x, t))\right\} .
$$

Note that since the variables in $|T|$ are included in both projections, the sets on the right-hand side are not disjoint. This motivates the following definition.

Definition 2.10. A dynamical system $(X, \varphi(x, t))$ is a join of dynamical systems $\left(X_{1}, \varphi_{1}\right)$, $\ldots,\left(X_{k}, \varphi_{k}\right)$ if there are projections $\pi_{i}: X \rightarrow X_{i}, i=1, \ldots, k$, such that the flow $\varphi(x, t)$ on $X$ admits a decomposition

$$
\{\varphi(x, t)\}=\bigcup_{i=1}^{k}\left\{\pi_{i}(\varphi(x, t))\right\}=: \bigcup_{i=1}^{k}\left\{\varphi_{i}(x, t)\right\} .
$$

If the ranges of the projections $\pi_{i}, i=1, \ldots, k$, are mutually disjoint, then $(X, \varphi(x, t))$ is a direct product of attractors $\left(X_{1}, \varphi_{1}\right), \ldots,\left(X_{k}, \varphi_{k}\right)$ and one can write $\varphi(x, t)=\left(\varphi_{1}(x, t), \ldots\right.$, $\left.\varphi_{k}(x, t)\right)$.

An invariant manifold $M$ (attractor $A$ ) is a join of manifolds $M_{1}, \ldots, M_{k}$ (attractors $\left.A_{1}, \ldots, A_{k}\right)$ if the dynamical system on $M(A)$ is a join of the corresponding dynamical systems on $M_{1}, \ldots, M_{k}$ (attractors $A_{1}, \ldots, A_{k}$ ). This definition, combined with the notion of join-irreducible elements of a lattice, will give us a minimal representation of the dynamics.

Definition 2.11. An element of a lattice is join-irreducible if the join (least upper bound) of $a$ and $b$, denoted $x=a \vee b$, implies $x=a$ or $x=b$. In other words these are elements that cannot be written as the join of two other elements of the lattice. An element is join-reducible if it is not join-irreducible.

Copyright (C) by SIAM. Unauthorized reproduction of this article is prohibited. 
Definition 2.12. A set of finite subsets $\left\{N_{1}, \ldots, N_{j}\right\}$ of a set $G$ will be called irredundant whenever all of its elements $N_{i}$ are mutually incomparable (with respect to the subset ordering).

Lemma 2.13 (see [17, Theorem 1.6.2]). Let $L$ be a distributive lattice. Then every $x \in L$ can be written uniquely as an irredundant join of join-irreducible elements.

Corollary 2.14. The filtrations $\mathbb{A}$ and $\mathbb{M}$ are fully characterized by the attractors (resp., manifolds) that correspond to join-irreducible elements in the lattice $U L_{\phi}$. Every attractor (resp., manifold) that corresponds to join reducible element $U \in U L_{\phi}$ is a join of attractors (resp., manifolds) corresponding to join-irreducible elements $V_{1}, \ldots, V_{k} \in U L_{\phi}$, for which

$$
U=V_{1} \vee V_{2} \vee \cdots \vee V_{k}
$$

is irredundant representation of $U$ in terms of $V_{1}, \ldots, V_{k}$.

Proof. By Lemma 2.13 each join-reducible element $U$ of the lattice $U L_{\phi}$ can be written as an irredundant join of join-irreducible elements. Since irredundant elements are not comparable in the lattice, in each $V_{j}$ there is a variable unique to this set that does not belong to any other set. On the other hand, since $U$ is a join of $V_{1}, \ldots, V_{k}$, each variable in $|U|$ is in one of the sets $\left|V_{1}\right|,\left|V_{2}\right|, \ldots,\left|V_{k}\right|$. Therefore the dynamical system generated by the variables in $|U|$ is a join of dynamical systems generated on the variables in $\left|V_{1}\right|,\left|V_{2}\right|, \ldots,\left|V_{k}\right|$. The result follows.

Until this point, our development was identical for both Takens' [20] (manifolds) and Sauer, Yorke, and Casdagli's [16] (attractors) formulations of the delay reconstruction process. Since the notion of a "large" set is different in these two approaches (generic in Takens versus prevalent in Sauer, Yorke, and Casdagli [16]), and the assumptions guaranteeing that the set of successful observation functions is large are different as well, we will formulate our theory separately for these two cases.

3. Extension of Takens' theorem. We start with a discussion of the technical restrictions we put on the dynamical system studied in this section. These assumptions come from Takens [20]. For a diffeomorphism $\phi: M \rightarrow M$ on a smooth, compact manifold $M$ of dimension $m$, we require the following properties, which are generic in $D^{2}(M, M)$, the space of twice differentiable diffeomorphisms on $M$ :

A1 The periodic points of $\phi$ with periods less than or equal to $2 m+1$ are finite in number.

A2 If $x$ is any periodic point with period $k \leq 2 m+1$, then the eigenvalues of the Jacobian $\left(D \phi^{k}\right)_{x}$ are all distinct and not equal to 1 .

For a smooth flow $\phi^{t}$ on a smooth, compact manifold $M$ of dimension $m$, and a time lag $T>0$, we impose the following properties, which are generic in $C^{2}(M, M) \times \mathbb{R}^{+}$(see Theorem A.1), where $C^{2}(M, M)$ is the space of twice differentiable flows on $M$ :

B1 The flow $\phi^{t}$ has no periodic orbits of period $k T$ for $k \leq 2 m+1$.

B2 If $\phi^{t}(x)=0$, the eigenvalues of the Jacobian $\left(D \phi^{T}\right)_{x}$ are distinct and not equal to 1 , where $\phi^{T}$ is the time $T$ flow-induced diffeomorphism.

We offer a proof in the appendix (Theorem A.1) that pairs $\left(\phi^{t}, T\right)$ satisfying B1 and B2 form an open and dense set in $C^{2}(M, M) \times \mathbb{R}^{+}$, where the choice of $T$ depends on the choice of $\phi^{t}$. In fact, we prove genericity under a less restrictive version of B1.

It is not clear a priori that conditions B1 and B2 are generic, in particular dense, within a class of flows with a fixed interaction graph $\mathcal{I} \mathcal{G}$, or a fixed component graph CG. Restriction

Copyright (c) by SIAM. Unauthorized reproduction of this article is prohibited. 
to such a class provides a smaller class of perturbations than the class of all smooth flows on $M$. To address this concern, we show in the appendix that conditions B1 and B2 are generic in the set of flows with a fixed component graph (Theorem A.6). This result does not (as far as we know) extend to the class of flows with a fixed interaction graph $\mathcal{I} \mathcal{G}$. Since we will later show that only strongly connected components of the interaction graph are recoverable using reconstruction methods, the lack of genericity of assumptions B1 and B2 for a fixed interaction graph does not restrict the generality of our result.

In order to apply Takens' theorem to dynamical subsystems $\phi_{U}^{t}$, we further assume the following:

C1 The elements $M_{U}$ of the invariant set filtration $\mathbb{M}$ are compact, invariant smooth manifolds.

This is not in general true, but it allows the application of Takens' theorem to the whole filtration, which we will henceforth call an invariant manifold filtration rather than an invariant set filtration.

3.1. Takens' theorems. We restate the results of Takens here, in slightly different language that is convenient for our purposes, and discuss the extension of his work to the current context.

Theorem 3.1 (Takens' theorem as restated by Huke $[9,10])$. Let $M$ be a smooth, compact manifold of dimension $m$. Let $\phi: M \rightarrow M$ be a diffeomorphism, $\phi \in D^{2}(M, M)$, that fulfills the generic properties $\mathrm{A} 1$ and $\mathrm{A} 2$. Then for generic $\varphi \in C^{2}(M, \mathbb{R})$, the map $\Phi_{(\phi, \varphi, 2 m)}: M \rightarrow$ $\mathbb{R}^{2 m+1}$ defined by

$$
\Phi_{(\phi, \varphi, 2 m)}(x)=\left(\varphi(x), \varphi(\phi(x)), \varphi\left(\phi^{2}(x)\right), \ldots, \varphi\left(\phi^{2 m}(x)\right)\right)
$$

is an embedding.

The main difference between this statement and the original Takens' theorem is that the conditions for a generic diffeomorphism are stated explicitly in the theorem body rather than in the proof. This allows one to choose a generic diffeomorphism first and then associate to it a generic set of observation functions $\varphi \in C^{2}(M, \mathbb{R})$.

When considering the analogous result for flows, we must make use of flow-defined diffeomorphisms.

Definition 3.2. For a flow $\phi^{t}$ on $M$, we define a diffeomorphism $\phi^{T}$ by fixing a time lag $T$. The composition of $\phi^{T}$ with itself, $\left(\phi^{T}\right)^{k}$, is identical to the value of the flow $\phi^{t}$ at $t=k T$.

Takens' Theorems 2 and 4 and Corollary 5 in [20] discuss the analogous result to Theorem 3.1 for flows. We have rewritten these results in the next theorems, in which we use the wording of Huke [9, 10] as in Theorem 3.1. We have done this to make explicit the requirements on the time lag $T$. In Takens' original formulation, he considers the case of $T=1$ first and then expands the result to generic $T>0$. Our formulation chooses a time lag $T$ together with the flow $\phi^{t}$.

Theorem 3.3 (Takens' Theorem 2 [20]). Let $\phi^{t}$ be a $C^{2}$ flow on a smooth, compact manifold $M$ of dimension $m$. Let $\left(\phi^{t}, T\right) \in C^{2}(M, M) \times \mathbb{R}^{+}$fulfill the generic conditions $\mathrm{B} 1$ and $\mathrm{B} 2$. Then for a generic observation function $\varphi \in C^{2}(M, \mathbb{R})$, the delay map

$$
\Phi_{\left(\phi^{T}, \varphi, 2 m\right)}(x)=\left(\varphi(x), \varphi\left(\phi^{T}(x)\right), \varphi\left(\left(\phi^{T}\right)^{2}(x)\right), \ldots, \varphi\left(\left(\phi^{T}\right)^{2 m}(x)\right)\right)
$$

is an embedding $\Phi_{\left(\phi^{T}, \varphi, 2 m\right)}: M \rightarrow \mathbb{R}^{2 m+1}$. 
The genericity of conditions B1 and B2 follows from the Kupka-Smale theorem [14] and the compactness of $M$. The proof is sketched in the appendix (see Theorem A.1), along with a proof of Theorem 3.3 assuming Theorem 3.1, which we do not prove, but refer the reader to $[9,10]$ and $[20]$.

The delay map $\Phi_{\left(\phi^{T}, \varphi, 2 m\right)}(x)$ preserves the dynamics of the flow $\phi^{t}$, as stated in Corollary 5 of Takens [20] for a time lag in a residual set of positive numbers, restated in different language below.

Theorem 3.4 (Takens' Theorem 4 and Corollary 5 [20]). Let $M$ and $m$ be as above. Let $p \in$ $M$ be an arbitrary point on the manifold. Then there exists a residual set $G(p) \subset C^{2}(M, M) \times$ $\mathbb{R}^{+}$such that if $\left(\phi^{t}, T\right) \in G(p)$, and if $\varphi \in C^{2}(M, \mathbb{R})$ is generic, then (1) the pair $\left(\phi^{t}, T\right)$ fulfills $\mathrm{B} 1$ and $\mathrm{B} 2$, and (2) the set of positive limit points of $\phi^{t}(p)$ is bijectively mapped to the limit points of the set $\left\{\Phi_{\left(\left(\phi^{T}\right)^{k}, \varphi, 2 m\right)}(p)\right\}_{k=0}^{\infty}$.

Remark. In Theorems 3.1 and 3.3, the concept of genericity means that a property holds on an open and dense set. In Theorem 3.4, genericity for the choice of a pair $\left(\phi^{t}, T\right)$ is reduced to a residual set - a countable intersection of open and dense sets.

In the appendix, we prove that conditions B1 and B2 hold generically for $\left(\phi^{t}, T\right)$ when the class of $\phi^{t}$ is restricted to those with a fixed component graph (Theorem A.6). This means that within the class of smooth flows satisfying the constraints $\pi_{V U} \circ \phi_{V}^{t}=\phi_{U}^{t} \circ \pi_{V U}$ for any pair of upper sets in $U L$ such that $U<V$, the pairs $\left(\phi^{t}, T\right)$ that fulfill conditions B1 and B2 form an open and dense set. Therefore we may apply Takens' theorem to each element of the manifold filtration.

Definition 3.5. For a fixed manifold $M$ and component graph $\mathrm{CG}$, we define a subset $\mathcal{P}_{\mathrm{CG}} \subset$ $C^{2}(M, M)$ as those flows where $\pi_{V U} \circ \phi_{V}^{t}=\phi_{U}^{t} \circ \pi_{V U}$ holds for any pair of upper sets $U<V$ in the lattice $U L$ induced by $C G$. Analogously, let $\mathcal{P}_{\mathrm{CG}} \subset D^{2}(M, M)$ be the set of diffeomorphisms such that $\pi_{V U} \circ \phi_{V}^{t}=\phi_{U}^{t} \circ \pi_{V U}$ holds for any pair of upper sets $U<V$.

Theorem 3.6. For a smooth, compact manifold $M$, a point $p \in M$, and a component graph CG, there exists a residual subset

$$
\mathrm{G}_{\mathcal{P}} \subset \mathcal{P}_{\mathrm{CG}} \times \mathbb{R}^{+} \subset C^{2}(M, M) \times \mathbb{R}^{+}
$$

such that each pair $\left(\phi^{t}, T\right) \in \mathrm{G}_{\mathcal{P}}$ satisfies the conclusions of Theorem 3.4.

Analogously for diffeomorphisms, given a smooth, compact manifold $M$ and a component graph CG, there is an open and dense set

$$
\mathrm{G}_{\mathcal{P}} \subset \mathcal{P}_{\mathrm{CG}} \subset D^{2}(M, M)
$$

such that $\phi \in \mathrm{G}_{\mathcal{P}}$ satisfies $\mathrm{A} 1$ and $\mathrm{A} 2$.

Proof. The proof of the statement for flows is in the appendix.

Remark. We have used the same notation $\mathcal{P}_{\mathrm{CG}}$ and $\mathrm{G}_{\mathcal{P}}$ for both diffeomorphisms and flows, although they belong to different spaces in the two different cases. The appropriate space should be clear from context.

It remains to show that the observation functions $\varphi$ can be chosen generically in a way that respects the dynamical structure.

Copyright $@$ by SIAM. Unauthorized reproduction of this article is prohibited. 
3.2. Filtration $\mathbb{Y}$ of observation functions. In this section we consider the filtration of spaces of observation functions with domains in $M_{U} \in \mathbb{M}$. Let $Y_{U}$ denote the set of observation functions of $M_{U},\left\{\varphi: \varphi \in C^{2}\left(M_{U}, \mathbb{R}\right)\right\}$, and let $Y:=\left\{\varphi: \varphi \in C^{2}(M, \mathbb{R})\right\}$ be the set of observation functions for the whole manifold. For $U \leq V$ in lattice $U L_{\phi}$, define inclusion $\iota_{U V}: Y_{U} \hookrightarrow Y_{V}$ as follows. For $\varphi \in Y_{U}$

$$
\iota_{U V}(\varphi):=\varphi \circ \pi_{V U}: M_{V} \rightarrow \mathbb{R}
$$

Thus $\iota_{U V}\left(Y_{U}\right)=\left\{\varphi \circ \pi_{V U}: M_{V} \rightarrow \mathbb{R} \mid \varphi \in Y_{U}\right\} \subset Y_{V}$ and $\iota_{U}\left(Y_{U}\right)=\left\{\varphi \circ \pi_{U}: M \rightarrow \mathbb{R} \mid \varphi \in\right.$ $\left.Y_{U}\right\} \subset Y$. The collection of sets $Y_{U}=\iota_{U}(Y)$ forms a filtration $\mathbb{Y}$ indexed by the set $U L_{\phi}$. This is depicted in Figure 6.

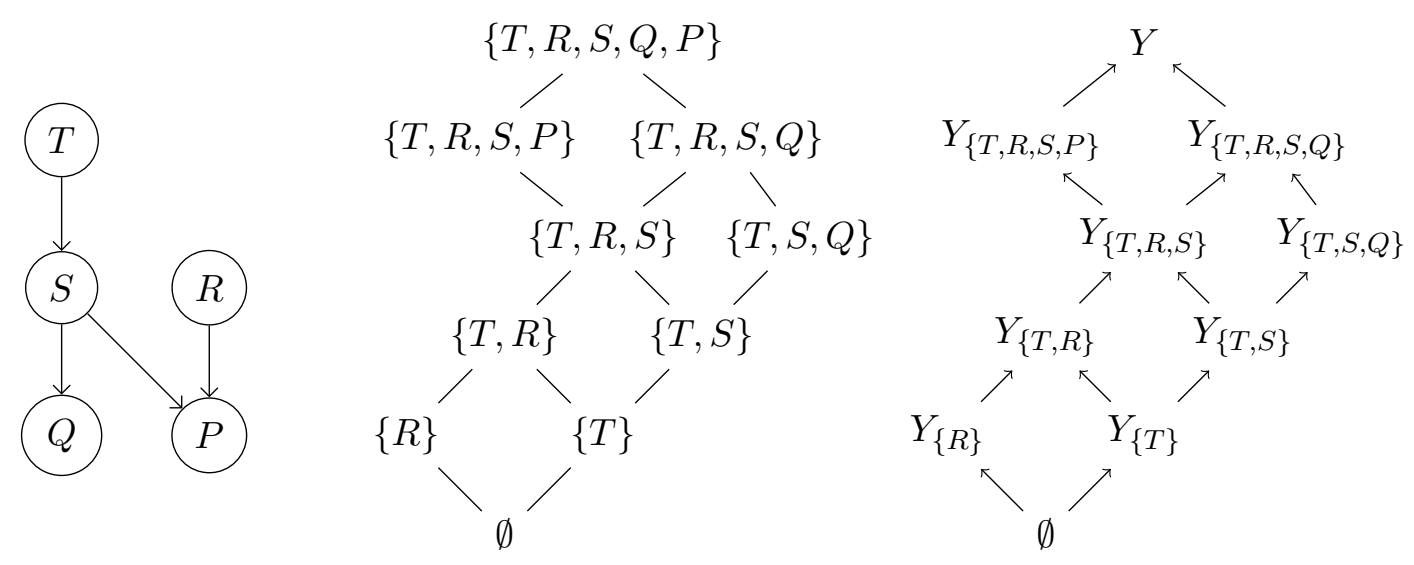

Figure 6. Example $\mathrm{CG}_{\phi}, U L_{\phi}$, and filtration of observation functions $\mathbb{Y}$.

A subset of the observation functions in $Y_{U}$ will be generic as an immediate consequence of Takens' theorem.

Corollary 3.7. Suppose that there is a generic diffeomorphism $\phi_{U} \in \mathrm{G}_{\mathcal{P}}$ or a generic flow $\left(\phi_{U}^{t}, T\right) \in \mathrm{G}_{\mathcal{P}}$ on a smooth, compact manifold $M_{U}$ that is a member of the filtration $\mathbb{M}$. Then by Takens' Theorem 3.1 or 3.3 , there exists a generic set $\mathcal{G}_{U} \subset Y_{U}=C^{2}\left(M_{U}, \mathbb{R}\right)$ of observation functions $\varphi$ such that the delay map induced by $\varphi$ is an embedding of $M_{U}$.

Remark. The generic set $\mathcal{G}_{U}$ depends either on the given diffeomorphism $\phi$ (under Theorem 3.1) or on the given flow $\phi^{t}$ and the choice of time lag $T$ (under Theorem 3.3). We omit these dependencies in the notation and note that we will assume the same time lag $T$ for every $\phi_{U}^{t}$ in the dynamical system.

We now discuss the relationship between the sets of observation functions $\mathcal{G}_{U}$ and $\mathcal{G}_{V}$ for $U<V$. Consider an observation function $\varphi: M_{U} \rightarrow \mathbb{R}$ that belongs to $\mathcal{G}_{U}$. Then, as noted above, $\varphi \circ \pi_{V U}: M_{V} \rightarrow \mathbb{R}$ is an observation in $Y_{V}$. However, in general $\varphi \circ \pi_{V U} \notin \mathcal{G}_{V}$. For instance, suppose $\varphi$ is a projection of $M_{U}$ onto some variable in $|U|$. Then one does not expect that observations of this variable will reconstruct the full dynamics of $M_{V}$ since no variable in $|V| \backslash|U|$ affects variables in $U$. In fact, although

$$
\iota_{U V}\left(\mathcal{G}_{U}\right)=\left\{\varphi \circ \pi_{V U}: M_{V} \rightarrow \mathbb{R} \mid \varphi \in \mathcal{G}_{U}\right\} \subset Y_{V}
$$


we expect that $\iota_{U V}\left(\mathcal{G}_{U}\right)$ belongs to the exceptional set $Y_{V} \backslash \mathcal{G}_{V}$. The reason is that when $\varphi_{V} \in$ $\mathcal{G}_{V}$, the map $\Phi_{\left(\phi^{T}, \varphi_{V}, 2 n_{V}\right)}$ reconstructs $\left(M_{V}, \phi_{V}\right)$, while when $\varphi_{U} \in \mathcal{G}_{U}$, the map $\Phi_{\left(\phi^{T}, \varphi_{U}, 2 n_{U}\right)}$ reconstructs $\left(M_{U}, \phi_{U}\right)$, which is a projection of $\left(M_{V}, \phi_{V}\right)$. If the projection $\pi_{V U}\left(M_{V}\right)=M_{U}$ is proper (i.e., not injective), we indeed have

$$
\iota_{U V}\left(\mathcal{G}_{U}\right) \subset Y_{V} \backslash \mathcal{G}_{V}
$$

The same argument shows that for any set $U$ that is a proper subset of $U_{\max }=\bigcup_{U \in U L} U$, we expect that

$$
\iota_{U}\left(\mathcal{G}_{U}\right) \subset Y \backslash \mathcal{G}_{\max },
$$

where $\mathcal{G}_{\max }$ is open and dense in $Y$, and $\varphi \in \mathcal{G}_{\max }$ provides an embedding of the manifold $M$. We formalize the requirement for (7) to hold in the following definition.

Definition 3.8. The dynamical system $(M, \phi)$ has a fully resolved manifold filtration $\mathbb{M}$ if the following two conditions are met:

1. Given comparable $U, V$ in the lattice $U L_{\phi}$ with $U<V$, the corresponding projection $\pi_{V U}: M_{V} \rightarrow M_{U}$ is noninjective.

2. Given noncomparable $U, V$ in the lattice $U L_{\phi}$, there exist no continuous surjections $f: M_{U} \rightarrow M_{V}$ or $g: M_{V} \rightarrow M_{U}$.

The first condition ensures that comparable, nonequal upper sets are associated with distinguishable invariant manifolds. In particular, the first condition is enough to ensure (7). As we will see later, the second condition is required to recover the full set of strongly connected components in CG.

We consider the union of the generic sets across the manifold filtration

$$
\mathcal{G}:=\bigcup_{U \in U L} \iota_{U}\left(\mathcal{G}_{U}\right)
$$

The set $\mathcal{G}$ is generic in $Y$, because it contains the open and dense set $\mathcal{G}_{\text {max }}$ along with some of its limit points. $\varphi \in \mathcal{G}$ may reconstruct any of the manifolds in the filtration.

Definition 3.9. We say that $\varphi$ is a proper observation function of $V$ with respect to $\mathcal{G}$ if

1. $\varphi \in Y_{V}$;

2. $\iota_{V}(\varphi) \in \mathcal{G}$;

3. and there is no $U<V$ with $\gamma \in Y_{U}$ such that $\iota_{U V}(\gamma)=\varphi$.

Corollary 3.10. Let $M$ be a smooth, compact manifold of dimension $m$, and let there be either a generic flow $\left(\phi^{t}, T\right) \in \mathrm{G}_{\mathcal{P}}$ or a generic diffeomorphism $\phi \in \mathrm{G}_{\mathcal{P}}$ as in Definition 3.5. Let the associated manifold filtration $\mathbb{M}$ be fully resolved and fulfill property $\mathrm{C} 1$. Let the generic set $\mathcal{G} \subset \bigcup Y_{U}$ be as in (8). Then if $\varphi$ is a proper observation function of $V$ with respect to $\mathcal{G}$, the delay reconstruction map

$$
\Phi_{\left(\phi^{T}, \varphi, k\right)}(x):=\left(\varphi(x), \varphi\left(\phi^{T}(x)\right), \ldots, \varphi\left(\left(\phi^{T}\right)^{k}(x)\right)\right)
$$

for flows or

$$
\Phi_{(\phi, \varphi, k)}(x):=\left(\varphi(x), \varphi(\phi(x)), \ldots, \varphi\left(\phi^{k}(x)\right)\right)
$$

for diffeomorphisms is an embedding of $M_{V}$ into $\mathbb{R}^{k+1}$ when $k \geq 2 n_{V}$.

Copyright ( $)$ by SIAM. Unauthorized reproduction of this article is prohibited. 
Proof. We need to show that $\varphi \in \mathcal{G}_{V}$; then the statement follows from Theorems 3.1 and 3.3. From the definition of $\mathcal{G}$ in (8) and the inclusion relation in (6), the assumptions $\varphi \in Y_{V}$ and $\iota_{V}(\varphi) \in \mathcal{G}$ imply that $\varphi \in \mathcal{G}_{V}$ or $\varphi \in \iota_{U V}\left(\mathcal{G}_{U}\right)$ for some $U<V$. These states cannot be simultaneously true because $\mathbb{M}$ is fully resolved, and so the inclusion image of the generic set in $U, \iota_{U V}\left(\mathcal{G}_{U}\right)$, is in the excluded set $Y_{V} \backslash \mathcal{G}_{V}$ for $U<V$. By assumption, there is no $\gamma \in \mathcal{G}_{U}$ such that $\iota_{U V}(\gamma)=\varphi$, thus $\varphi \in \mathcal{G}_{V}$.

Corollary 3.10 partitions the set of observation functions $Y$. Consider an observation function $\varphi \in Y$ such that there exists $\gamma \in Y_{U}$ with $\iota_{U}(\gamma)=\varphi$. If $\varphi$ is in the generic set $\mathcal{G}$ and the conditions of Corollary 3.10 are met, then $\varphi$ provides a reconstruction of $M_{U}$. In particular, if $U=U_{\max }$, then $\varphi \in \mathcal{G}_{\max }$ reconstructs a diffeomorphism to $M$; if $U<U_{\max }$, then $\varphi \in \mathcal{G} \backslash \mathcal{G}_{\max }\left(\right.$ or $\gamma \in \mathcal{G}_{U}$ ) reconstructs a diffeomorphism to $M_{U}$; and if $\varphi \in Y \backslash \mathcal{G}$ (or $\left.\gamma \notin \mathcal{G}_{U}\right)$, then it does not reconstruct $M_{U}$, although its reconstruction may be diffeomorphic to some other $M_{V}$.

3.3. Homeomorphisms vs. projections. Theorems 3.1 and 3.3 provide a basis for identifying dynamical drivers in deterministic systems when applied to the filtration structure $\mathbb{M}$ of the system. In this section we present our major result, a theorem that suggests an algorithm for recovering the transitive closures of the interaction and component graphs of the dynamical system. At this point, we will relax the idea of diffeomorphic manifolds to the notion of topological equivalence (see, for example, Arnol'd [1]). Two topologically equivalent systems have homeomorphic phase spaces, rather than diffeomorphic phase spaces. The presence or absence of homeomorphisms between manifolds in $\mathbb{M}$ is the property that allows the recovery of dynamical relationships.

Theorem 3.11. Let $M$ be a smooth, compact manifold of dimension $m$, and let there be either a generic flow $\left(\phi^{t}, T\right) \in \mathrm{G}_{\mathcal{P}}$ or a generic diffeomorphism $\phi \in \mathrm{G}_{\mathcal{P}}$. Let the associated manifold filtration $\mathbb{M}$ be fully resolved and fulfill property $\mathrm{C} 1$. Let $\mathcal{G}$ be the generic set of observation functions defined by (8). Consider a proper observation function $\varphi_{1}$ of $U$, and a proper observation function $\varphi_{2}$ of $V$. Denote the reconstructions $M_{i}:=\Phi_{\left(\phi^{T}, \varphi_{i}, k_{i}\right)}(M)$ for flows or $M_{i}:=\Phi_{\left(\phi, \varphi_{i}, k_{i}\right)}(M)$ for diffeomorphisms, $i=1,2$, with $k_{1} \geq 2 n_{U}$ and $k_{2} \geq 2 n_{V}$. Then the following hold:

(a) $U=V$ (i.e., both observation functions are from the same class $\mathcal{G}_{U}$ ) if and only if there is a homeomorphism

$$
\Psi_{1,2}: M_{1} \rightarrow M_{2}
$$

(b) $U<V$ if and only if there is a continuous surjective, noninjective map

$$
\Pi_{2,1}: M_{2} \rightarrow M_{1}
$$

Proof. In the following, we will use the notation for a flow, with the understanding that the proof holds for either flows or diffeomorphisms.

(a) Forward direction: As $U=V$ we will only use $U$. By Corollary 3.10, $\Phi_{\left(\phi^{T}, \varphi_{1}, k_{1}\right)}$ : $M_{U} \rightarrow M_{1}$ and $\Phi_{\left(\phi^{T}, \varphi_{2}, k_{2}\right)}: M_{U} \rightarrow M_{2}$ are embeddings, thus diffeomorphisms onto their images. Defining $\Psi_{1,2}:=\Phi_{\left(\phi^{T}, \varphi_{2}, k_{2}\right)} \circ \Phi_{\left(\phi^{T}, \varphi_{1}, k_{1}\right)}^{-1}$ gives a diffeomorphism, and therefore a homeomorphism, $M_{1} \rightarrow M_{2}$, as shown in Figure 7(a). 


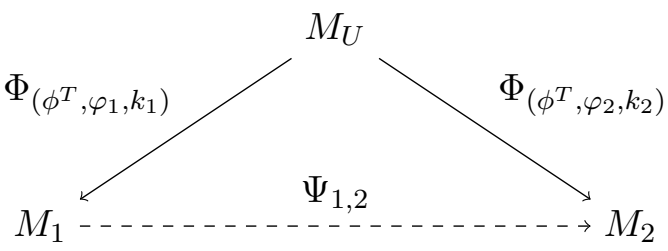

(a)

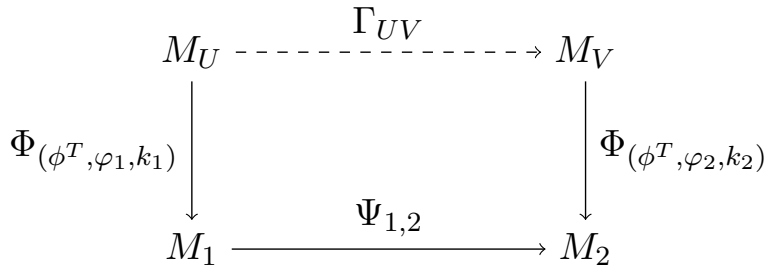

(b)

Figure 7. (a) Induced homeomorphism $\Psi_{1,2}$ when $U=V$. (b) Induced homeomorphism $\Gamma_{U V}$ when $\Psi_{1,2}$ exists.

Reverse direction: Assume that there exists a homeomorphism $\Psi_{1,2}: M_{1} \rightarrow M_{2}$, and the relationship between $M_{U}$ and $M_{V}$ is unknown. As in the forward direction, the maps $\Phi_{\left(\phi^{T}, \varphi_{1}, k_{1}\right)}: M_{U} \rightarrow M_{1}$ and $\Phi_{\left(\phi^{T}, \varphi_{2}, k_{2}\right)}: M_{V} \rightarrow M_{2}$ are diffeomorphisms. So the map

$$
\Gamma_{U V}:=\Phi_{\left(\phi^{T}, \varphi_{2}, k_{2}\right)}^{-1} \circ \Psi_{1,2} \circ \Phi_{\left(\phi^{T}, \varphi_{1}, k_{1}\right)}
$$

constructed from Figure $7(\mathrm{~b})$ is a homeomorphism $M_{U} \rightarrow M_{V}$. Since $(M, \phi)$ has a fully resolved manifold structure, the existence of the homeomorphism $\Gamma_{U V}$ ensures that $U$ and $V$ are comparable and that $U$ and $V$ are equal.

(b) Forward direction: Now suppose $U<V$, so that the noninjective projection $\pi_{V U}: M_{V} \rightarrow$ $M_{U}$ exists. As above, $\Phi_{\left(\phi^{T}, \varphi_{1}, k_{1}\right)}$ and $\Phi_{\left(\phi^{T}, \varphi_{2}, k_{2}\right)}$ are diffeomorphisms of $M_{U}$ and $M_{V}$, respectively. Then

$$
\Pi_{2,1}:=\Phi_{\left(\phi^{T}, \varphi_{1}, k_{1}\right)} \circ \pi_{V U} \circ \Phi_{\left(\phi^{T}, \varphi_{2}, k_{2}\right)}^{-1}: M_{2} \rightarrow M_{1}
$$

is the desired noninjective surjection (Figure 8(a)).

Reverse direction: Now suppose the noninjective, surjective map $\Pi_{2,1}: M_{2} \rightarrow M_{1}$ exists. The diffeomorphic maps $\Phi_{\left(\phi^{T}, \varphi_{1}, k_{1}\right)}$ and $\Phi_{\left(\phi^{T}, \varphi_{2}, k_{2}\right)}$ induce a noninjective projection

$$
\bar{\Pi}_{V U}:=\Phi_{\left(\phi^{T}, \varphi_{1}, k_{1}\right)}^{-1} \circ \Pi_{2,1} \circ \Phi_{\left(\phi^{T}, \varphi_{2}, k_{2}\right)}: M_{V} \rightarrow M_{U}
$$

as in Figure 8(b). Since $\mathbb{M}$ is fully resolved, the existence of $\Pi_{2,1}$ ensures that $U$ and $V$ are comparable, and since the map $\bar{\Pi}_{V U}$ is noninjective, it must be true that $V>U$.

Theorem 3.11 implies that two variables reconstruct homeomorphic projections of $M$ only if they belong to the same strongly connected component in $\mathrm{CG}_{\phi}$. We now propose an algorithm for establishing interactions between variables and prove that the transitive closures of the interaction and component graphs of the dynamical system can be recovered from the output of the algorithm.

\subsection{Algorithm.}

Algorithm 1.

1. Let the set of variables be the set of vertices in a graph that we will call $R G$.

2. For every variable $x$, build the reconstructed manifold $M_{x}:=\Phi_{\left(\phi^{T}, \varphi_{x}, k\right)}(M)$, where $\varphi_{x}$ depends only on $x$ and $k$ is large enough to work for the whole filtration. 


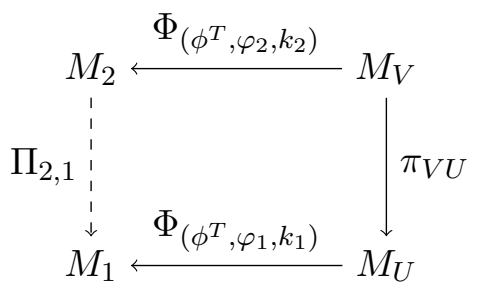

(a)

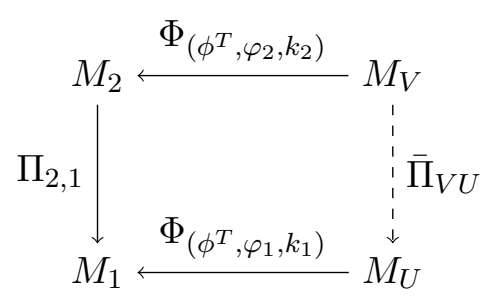

(b)

Figure 8. (a) Induced projection $\Pi_{2,1}: M_{2} \rightarrow M_{1}$ when $U<V$. (b) Induced projection $\bar{\Pi}_{V U}: M_{V} \rightarrow M_{U}$ when $\Pi_{2,1}$ exists.

3. For every pair $x$ and $y$, test, using some criterion, whether there exists a continuous surjection $f: M_{x} \rightarrow M_{y}$, and if there exists a continuous surjection $g: M_{y} \rightarrow M_{x}$ such that if $f$ and $g$ are injective, $f^{-1}=g$.

4. If $f$ exists, draw an edge $y \rightarrow x$ in $R G$, and if $g$ exists, draw an edge $x \rightarrow y$.

Definition 3.12. We say that $U \subset U L_{\phi}$ is the minimal upper set of $x \in|U|$ if for every $V<U$ we have $x \notin|V|$. We denote a minimal upper set with a subscript of $\min : x \in\left|U_{\min }\right|$.

Theorem 3.13. Let $M$ be a smooth, compact manifold of dimension $m$, and let there be either a generic flow $\left(\phi^{t}, T\right) \in \mathrm{G}_{\mathcal{P}}$ or a generic diffeomorphism $\phi \in \mathrm{G}_{\mathcal{P}}$. Let the associated manifold filtration $\mathbb{M}$ be fully resolved and fulfill property $\mathrm{C} 1$. Further assume that given $x \in\left|U_{\min }\right|$, there exists a generic observation function $\varphi_{x} \in \mathcal{G}_{U}$, which depends only on observations of $x$. Then the following hold:

1. The graph $R G$ constructed by the algorithm is the transitive closure of $\mathcal{I} \mathcal{G}$, less any self-loops.

2. The graph of strongly connected path components of $R G$ is the transitive closure of the component graph CG.

Proof.

1. By construction, the graph $R G$ has the same set of vertices as $\mathcal{I G}$. Self-loops cannot be resolved by the algorithm, since $M_{x}$ is always homeomorphic to itself regardless of the presence or absence of a self-loop at $x$ in $\mathcal{I} \mathcal{G}$.

Let $x \in\left|U_{\min }\right|, y \in\left|V_{\min }\right|$, and $w \in\left|W_{\min }\right|$ be arbitrary variables in the set of vertices, with $x \neq y$ and $x \neq w$. The generic maps $\varphi_{x} \in \mathcal{G}_{U}, \varphi_{y} \in \mathcal{G}_{V}$, and $\varphi_{w} \in \mathcal{G}_{W}$ exist as assumed. Note that $\mathbb{M}$ fully resolved, $x \in\left|U_{\text {min }}\right|$, and $\varphi_{x} \in \mathcal{G}_{U}$ all together imply that $\varphi_{x}$ is a proper observation function with respect to $\mathcal{G}$. Similarly for $\varphi_{y}$ and $\varphi_{w}$. Then by Corollary 3.10, each of these induces a reconstruction that is diffeomorphic to the associated element of the manifold filtration $\mathbb{M}$; that is, $M_{x}=\Phi_{\left(\phi^{T}, \varphi_{x}, k_{x}\right)}(M)$ is diffeomorphic to $M_{U}$ and so forth.

Suppose that there is an edge in $\mathcal{I G}$ from $x \in\left|U_{\min }\right|$ to $y \in\left|V_{\min }\right|$. Then $U$ and $V$ have the relation $U \leq V$ by the construction of $U L_{\phi}$. By Theorem 3.11, if $U=V$, then the reconstructions $M_{x}$ and $M_{y}$ are homeomorphic; hence there is an edge from $x$ to $y$ in the graph $R G$. If instead $U<V$, then there is a continuous surjective map from $M_{y}$ to $M_{x}$, and so there is an edge from $x$ to $y$ in $R G$.

Now suppose there is an edge from $x \in\left|U_{\min }\right|$ to $w \in\left|W_{\min }\right|$ in $R G$ that does not exist 
in $\mathcal{I} \mathcal{G}$. By the algorithm, there must be a continuous surjection from $M_{w}$ to $M_{x}$. By Theorem 3.11, $U \leq W$, which implies that there is a path from any variable in $|U|$ to any variable in $|W|$ along directed edges in $\mathcal{I} \mathcal{G}$. Thus the edge $x \rightarrow w$ is an element of the transitive closure of $\mathcal{I} \mathcal{G}$.

2. This follows from 1 .

We discuss a specific implementation of Algorithm 1 in section 5. In the following section, we present the analogous theorems and algorithm for compact attractors instead of compact manifolds, based on the fractal delay embedding prevalence theorem of Sauer, Yorke, and Casdagli [16].

4. Extension to Attractors. To obtain similar results for attractors, the conditions which the dynamical system must obey are slightly different. We now discuss these technical restrictions. For a diffeomorphism, $\phi: \mathbb{R}^{k} \rightarrow \mathbb{R}^{k}$, a compact subset $A \subset \mathbb{R}^{k}$ of box-counting dimension $d$ that is invariant under $\phi$, and $n>2 d$, we require the following:

A1 For every positive integer $p \leq n$, the set of periodic points of period $p$ has box-counting dimension less than $\frac{p}{2}$.

A2 The linearization $D \phi^{p}$ for each of these orbits has distinct eigenvalues.

Consider a flow $\phi^{t}$ on $\mathbb{R}^{k}$ and let $A$ be a compact subset of $\mathbb{R}^{k}$ of box-counting dimension $d$ that is invariant under $\phi$. Let $n>2 d$ and $T>0$. Assume the following:

B1 $A$ contains at most a finite number of equilibria.

B2 There are no periodic orbits of period $T$ or $2 T$, and there are at most finitely many periodic orbits of period $3 T, 4 T, \ldots, n T$.

B3 The linearizations of those periodic orbits have distinct eigenvalues.

We conjecture, similar to our result in Theorem A.6, that these assumptions are prevalent (as described below) in the set of flows $\phi^{t}$ or diffeomorphisms $\phi$ restricted to a fixed component graph CG. The proof remains open. In the proofs below, we assume that if A1 and A2 hold for $\phi$ or B1-B3 hold for $\phi^{t}$, they also hold for all $\phi_{U}$ or $\phi_{U}^{t}$, where $U$ is a set in the upper set lattice $U L$.

4.1. Observation function filtration for attractors. In this section we phrase the work of Sauer, Yorke, and Casdagli [16] in our context. The importance of the results presented in [16] is that the invariant set of a dynamical system may not be a compact smooth manifold. In this setting, Sauer, Yorke, and Casdagli [16] replace Takens' notion of genericity of observations of dynamics on compact manifolds with a notion of "almost everywhere," or probability one, for observations of dynamics on compact attractors. It is not obvious how to generalize the notion of probability from a finite-dimensional space to an infinite-dimensional space, and the notion of prevalence [16] has been developed to address this issue.

Definition 4.1. A Borel subset $S$ of a normed linear space $V$ is prevalent if there is a finitedimensional subspace $E$ of $V$ such that for each $v \in V, v+e$ belongs to $S$ for (Lebesgue) almost every $e$ in $E$.

We will proceed by considering the filtration of spaces of observation functions for each entry in the upper set lattice $U L_{\phi}$. In this section, $Y_{U}$ denotes the set of smooth observation functions $Y_{U}:=\left\{\varphi: A_{U} \rightarrow \mathbb{R}\right\}$ with $\varphi \in C^{1}$ (this is relaxed from Takens' theorem, in which $\varphi \in C^{2}$ is required). The sets $Y_{U}$ and $Y=\bigcup_{U} \iota_{U}\left(Y_{U}\right)$ form a filtration $\mathbb{Y}$, as discussed in the previous section. 
We restate the fractal delay embedding prevalence theorem of [16] in our context, which is analogous to Theorems 3.1 and 3.3 in the previous section.

Theorem 4.2 (Sauer, Yorke, and Casdagli [16]). Let $\phi: \mathbb{R}^{n} \rightarrow \mathbb{R}^{n}$ be a diffeomorphism satisfying the assumptions $\mathrm{A} 1$ and $\mathrm{A} 2$ above, or a flow satisfying B1-B3. For every observation function $\varphi$ in a prevalent subset $\mathcal{P}_{U} \subset Y_{U}$ with $T>0$, the delay reconstruction map of $A_{U}$,

$$
\Phi_{\left(\phi^{T}, \varphi, k\right)}(x):=\left(\varphi(x), \varphi\left(\phi^{T}(x)\right), \ldots, \varphi\left(\left(\phi^{T}\right)^{k}(x)\right)\right)
$$

for flows or

$$
\Phi_{(\phi, \varphi, k)}(x):=\left(\varphi(x), \varphi(\phi(x)), \ldots, \varphi\left(\phi^{k}(x)\right)\right)
$$

for diffeomorphisms, is

1. one-to-one, and

2. an immersion on each compact subset $C_{U}$ of a smooth manifold contained in $A_{U}$, provided that $k \geq 2$ boxdim $\left(A_{U}\right)$, the box-counting dimension of $A_{U}$.

For the remainder of the work on attractors, a set $C_{U} \subset M_{U} \subset A_{U}$ will mean a compact subset of a smooth manifold contained in $A_{U}$. The second item in Theorem 4.2 states that $\Phi_{\left(\phi^{T}, \varphi, k\right)}$ restricted to $C_{U}$ is differentiable with an injective derivative onto its image $\Phi_{\left(\phi^{T}, \varphi, k\right)}\left(C_{U}\right) \subset \mathbb{R}^{k+1}$. The following corollary gives us a result, which is compatible with the attractor filtration and more suitable for applications since it replaces differentiable functions with a homeomorphism.

Corollary 4.3. Let $\phi: \mathbb{R}^{n} \rightarrow \mathbb{R}^{n}$ be a diffeomorphism satisfying the assumptions $\mathrm{A} 1$ and A2 above, or a flow satisfying B1-B3. Let $C_{U} \subset M_{U} \subset A_{U}$, let $\varphi \in \mathcal{P}_{U}$, and let $\Phi_{\left(\phi^{T}, \varphi, k\right)}$ or $\Phi_{(\phi, \varphi, k)}$ be the delay reconstruction map from Theorem 4.2 into $\mathbb{R}^{k+1}$. Then the delay map $\Phi_{\left(\phi^{T}, \varphi, k\right)}$ or $\Phi_{(\phi, \varphi, k)}$ restricted to $C_{U}$ is a homeomorphism onto its image.

Proof. Let $C_{I}$ denote the image of $C_{U}$ under the delay map, and let $H_{\Phi, C_{U}}$ denote the restricted map $H_{\Phi, C_{U}}: C_{U} \rightarrow C_{I}$. By Theorem 4.2, $H_{\Phi, C_{U}}$ is a differentiable surjection onto its image and is also one-to-one, and therefore a differentiable bijection. To be a homeomorphism, we need only that $H_{\Phi, C_{U}}^{-1}$ be continuous, which follows from a theorem in Munkres [13]. We repeat the brief argument here. Let $D$ be an arbitrary closed subset of $C_{U}$. Since $C_{U}$ is compact and $D$ is closed, $D$ is compact. Then $H_{\Phi, C_{U}}$ continuous implies $H_{\Phi, C_{U}}(D)$ is a compact subset of $C_{I}$. Since $C_{I} \subset \mathbb{R}^{k+1}, C_{I}$ is Hausdorff, which implies that the compact subset $H_{\Phi, C_{U}}(D)$ is closed. Since the inverse image of an arbitrary closed subset in the range of $H_{\Phi, C_{U}}^{-1}$ is closed, $H_{\Phi, C_{U}}^{-1}$ is continuous.

In order to apply Corollary 4.3 to $\mathbb{A}$, we need the idea of a fully resolved attractor structure, analogous to Definition 3.8. It is useful to introduce the notation $\bigcup M_{U}$, denoting the union of all smooth manifolds in the attractor $A_{U}$.

Definition 4.4. The dynamical system $(A, \phi)$ has a fully resolved attractor filtration $\mathbb{A}$ if the following two conditions are met:

1. Given comparable $U, V$ in the lattice $U L_{\phi}$ with $U<V$, the corresponding projection $\pi_{V U}: A_{V} \rightarrow A_{U}$ is noninjective and is a surjection from $\bigcup M_{V} \subset A_{V}$ to $\bigcup M_{U} \subset A_{U}$.

2. Given noncomparable $U, V$ in the lattice $U L_{\phi}$, there is no surjection $f: \bigcup M_{U} \rightarrow \bigcup M_{V}$ that is also continuous when restricted to any compact subset $C_{U} \subset M_{U} \subset A_{U}$.

As in the previous section, the first condition ensures that comparable, nonequal upper sets are associated with distinguishable attractors by the relation $\iota_{U V}\left(\mathcal{P}_{U}\right) \subset Y_{V} \backslash \mathcal{P}_{V}$, and the

Copyright $\odot$ by SIAM. Unauthorized reproduction of this article is prohibited. 
second condition is required to recover the full set of strongly connected components in CG. Additionally, the requirement in the first condition that smooth manifolds map to smooth manifolds is analogous to assumption $\mathrm{C} 1$ in section 3.

Similar to Corollary 3.10 we have the following.

Corollary 4.5. Let $\phi: \mathbb{R}^{n} \rightarrow \mathbb{R}^{n}$ be a diffeomorphism satisfying the assumptions $\mathrm{A} 1$ and $\mathrm{A} 2$ above, or a flow satisfying $\mathrm{B} 1-\mathrm{B} 3$. Let $\mathbb{A}$ be the attractor filtration, and let $\mathbb{Y}$ be the filtration of observation functions associated to $\phi$. Assume $\mathbb{A}$ is fully resolved. Then there exists a prevalent set $\mathcal{P} \subset Y$ such that if $\varphi$ is a proper observation function of $V$ with respect to $\mathcal{P}$, then the delay reconstruction map $\Phi_{\left(\phi^{T}, \varphi, k\right)}(x)$ or $\Phi_{(\phi, \varphi, k)}(x)$ is one-to-one on $A_{V}$ and a homeomorphism from any $C_{V} \subset M_{V} \subset A_{V}$ onto its image in $\mathbb{R}^{k+1}$ when $T>0$ and $k \geq 2$ $\operatorname{box} \operatorname{dim}\left(A_{V}\right)$.

Proof. Analogously to (8) we define a set

$$
\mathcal{P}:=\bigcup_{U \in U L} \iota_{U}\left(\mathcal{P}_{U}\right)
$$

The proof that $\varphi \in \mathcal{P}_{V}$ is the same as Corollary 3.10, because it depends only on $\iota_{U V}\left(\mathcal{P}_{U}\right) \subset$ $Y_{V} \backslash \mathcal{P}_{V}$, which has an exact analogue for manifolds. Since $\varphi \in \mathcal{P}_{V}$, the result follows directly from the application of Theorem 4.2 and Corollary 4.3.

4.2. Homeomorphisms vs. projections on subsets of attractors. We present our major result for attractors, a theorem analogous to Theorem 3.11 that suggests an algorithm for recovering the interactions between variables in a dynamical system. The proof is quite similar to that of Theorem 3.11, and the commuting diagrams in that proof may be useful to the reader here.

Theorem 4.6. Consider a dynamical system $\phi$ that is either a diffeomorphism satisfying $\mathrm{A} 1$ and $\mathrm{A} 2$, or a flow satisfying $\mathrm{B} 1-\mathrm{B} 3$, its attractor $A$, associated attractor filtration $\mathbb{A}$, and observation filtration $\mathbb{Y}$. Assume that $\mathbb{A}$ is fully resolved. Let $\mathcal{P}$ be the prevalent set of observation functions guaranteed by Corollary 4.5. Let $\varphi_{1}$ be a proper observation function of $U$, and let $\varphi_{2}$ be a proper observation function of $V$. Let $T>0$ be the chosen time lag for the flow. Let $A_{i}:=\Phi_{\left(\phi^{T}, \varphi_{i}, k_{i}\right)}(A)$ for flows or $A_{i}:=\Phi_{\left(\phi, \varphi_{i}, k_{i}\right)}(A)$ for diffeomorphisms, $i=1,2$, with $k_{1} \geq 2 \operatorname{boxdim}\left(A_{U}\right)$ and $k_{2} \geq 2 \operatorname{boxdim}\left(A_{V}\right)$. Let $C_{U}$ and $C_{V}$ be arbitrary compact subsets of manifolds of $A_{U}$ and $A_{V}$, respectively, and define their images as $C_{1}$ and $C_{2}$, respectively, under the delay map. Furthermore let $\bigcup M_{1} \subset A_{1}$ denote the union of images of the smooth manifolds of $A_{U}$, and likewise for $\bigcup M_{2} \subset A_{2}$. Then the following hold:

(a) $U=V$ (i.e., both observation functions are from the same class $\mathcal{P}_{U}$ ) if and only if there is an injective map

$$
\Psi_{1,2}: A_{1} \rightarrow A_{2}
$$

that is a bijection from $\bigcup M_{1} \subset A_{1}$ to $\bigcup M_{2} \subset A_{2}$ and a homeomorphism when restricted to any $C_{1}$.

(b) $U<V$ if and only if there is a noninjective map

$$
\Pi_{2,1}: A_{2} \rightarrow A_{1}
$$

that is a surjection from $\bigcup M_{2}$ to $\bigcup M_{1}$ and continuous when restricted to any $C_{2}$.

Copyright ( $)$ by SIAM. Unauthorized reproduction of this article is prohibited. 
Proof. As in the previous section, we will use only the notation for flows, noting that the proof holds when substituting the notation for diffeomorphisms.

(a) Forward direction: As $U=V$ we will only use $U$. By Corollary $4.5, \Phi_{\left(\phi^{T}, \varphi_{1}, k_{1}\right)}$ and $\Phi_{\left(\phi^{T}, \varphi_{2}, k_{2}\right)}$ are one-to-one on $A_{U}$ and homeomorphisms on any $C_{U}$. Then the composition map $\Psi_{1,2}:=\Phi_{\left(\phi^{T}, \varphi_{2}, k_{2}\right)} \circ \Phi_{\left(\phi^{T}, \varphi_{1}, k_{1}\right)}^{-1}: A_{1} \rightarrow A_{2}$ is injective, and is a homeomorphism on any $C_{1} \rightarrow C_{2}$, where $C_{1}$ and $C_{2}$ are both images of $C_{U}$. Notice that $\Phi_{\left(\phi^{T}, \varphi_{1}, k_{1}\right)}$ and $\Phi_{\left(\phi^{T}, \varphi_{2}, k_{2}\right)}$ are not guaranteed continuous on $\bigcup M_{U}$, but since $\Phi_{\left(\phi^{T}, \varphi_{1}, k_{1}\right)}$ and $\Phi_{\left(\phi^{T}, \varphi_{2}, k_{2}\right)}$ are bijective on $\bigcup M_{U}, \Psi_{1,2}$ is a bijection from $\bigcup M_{1}$ to $\bigcup M_{2}$.

Reverse direction: We assume that there exists a one-to-one map $\Psi_{1,2}: A_{1} \rightarrow A_{2}$ that is bijective on $\bigcup M_{1}$ and a homeomorphism when restricted to $C_{1}$. As above, by Corollary 4.5, $\Phi_{\left(\phi^{T}, \varphi_{1}, k_{1}\right)}$ and $\Phi_{\left(\phi^{T}, \varphi_{2}, k_{2}\right)}$ are one-to-one on $A_{U}$ and $A_{V}$, and are homeomorphisms on any $C_{U}$ and $C_{V}$. Then the map

$$
\Gamma_{U V}:=\Phi_{\left(\phi^{T}, \varphi_{2}, k_{2}\right)}^{-1} \circ \Psi_{1,2} \circ \Phi_{\left(\phi^{T}, \varphi_{1}, k_{1}\right)}: A_{U} \rightarrow A_{V}
$$

is injective on $A_{U}$, bijective on $\bigcup M_{U}$, and a homeomorphism on any $C_{U}$. Since $\mathbb{A}$ is fully resolved, $U$ and $V$ are comparable because $\Gamma_{U V}$ exists, and $U=V$ because $\Gamma_{U V}$ is injective.

(b) Forward direction: Suppose $U<V$, so that the noninjective, continuous projection (surjection) $\pi_{V U}: A_{V} \rightarrow A_{U}$ exists. $\pi_{V U}$ maps the compact sets $C_{V} \subset M_{V} \subset A_{V}$ surjectively to the compact sets $C_{U} \subset M_{U} \subset A_{U}$ by assumption. Since the maps $\Phi_{\left(\phi^{T}, \varphi_{1}, k_{1}\right)}$ and $\Phi_{\left(\phi^{T}, \varphi_{2}, k_{2}\right)}$ are injective on $A_{U}$ and $A_{V}$, their inverses exist, leading to the well-defined map

$$
\Pi_{2,1}:=\Phi_{\left(\phi^{T}, \varphi_{1}, k_{1}\right)} \circ \pi_{V U} \circ \Phi_{\left(\phi^{T}, \varphi_{2}, k_{2}\right)}^{-1}: A_{2} \rightarrow A_{1},
$$

which is noninjective. Furthermore, $\Pi_{2,1}$ maps $C_{2} \rightarrow C_{V} \rightarrow C_{U} \rightarrow C_{1}$ in a composition of continuous surjections; i.e., it is a continuous surjection itself on $C_{2}$. As proved in part (a), $\Pi_{2,1}$ is not continuous on the union $\bigcup M_{2}$, but it is a surjection onto $\bigcup M_{1}$.

Reverse direction: Now suppose the noninjective map $\Pi_{2,1}: A_{2} \rightarrow A_{1}$ exists, is a surjection from $\bigcup M_{2}$ to $\bigcup M_{1}$, and is continuous on any $C_{2}$. Since $\Phi_{\left(\phi^{T}, \varphi_{1}, k_{1}\right)}$ and $\Phi_{\left(\phi^{T}, \varphi_{2}, k_{2}\right)}$ are injective, their inverses exist, so the map

$$
\bar{\Pi}_{V U}:=\Phi_{\left(\phi^{T}, \varphi_{1}, k_{1}\right)}^{-1} \circ \Pi_{2,1} \circ \Phi_{\left(\phi^{T}, \varphi_{2}, k_{2}\right)}: A_{V} \rightarrow A_{U}
$$

is well defined and noninjective via $\Pi_{2,1} . \bar{\Pi}_{V U}$ is a continuous surjection on any $C_{2}$, implying that $\bar{\Pi}_{V U}$ is a surjection from $\bigcup M_{2}$ to $\bigcup M_{1}$. Since $\mathbb{A}$ is fully resolved, the existence of $\bar{\Pi}_{V U}$ ensures that $U$ and $V$ are comparable, and since the map $\bar{\Pi}_{V U}$ is noninjective, it must be true that $V>U$.

The notion of fully resolved attractor filtration reflects not only a desire to have distinct attractors associated to different upper sets in the lattice $U L$, but also the need for these characteristics to be computable, at least approximately, from finite data. For two reconstructions of the same attractor in a fully resolved system, Theorem 4.6 asserts the existence of a map that is a homeomorphism when restricted to compact subsets of smooth manifolds in an attractor. That is, there are homeomorphisms between reconstructions of compact sets $C_{1}$ and $C_{2}$ that have observation functions from the same prevalent set. It is difficult to verify such a 
condition computationally since we do not know all smooth manifolds that are embedded in the attractor. Instead, we only concentrate on the collection of unstable manifolds of points in the attractor, since these are embedded manifolds in the attractor. We denote this collection as $\bigcup_{a} W_{U}^{u}(a) \subset A_{U}$, where $a \in A_{U}$.

Definition 4.7. The dynamical system $(A, \phi)$ has a strongly resolved attractor filtration $\mathbb{A}$ if the following two conditions are met:

1. Given comparable $U, V$ in the lattice $U L_{\phi}$ with $U<V$, the corresponding projection $\pi_{V U}: A_{V} \rightarrow A_{U}$ is a noninjective surjection from $\bigcup_{b} W_{V}^{u}(b) \subset A_{V}$ to $\bigcup_{a} W_{U}^{u}(a) \subset A_{U}$.

2. Given noncomparable $U, V$ in the lattice $U L_{\phi}$, there is no surjection $f: \bigcup_{a} W_{U}^{u}(a) \rightarrow$ $\bigcup_{b} W_{V}^{u}(b)$ that is also continuous when restricted to any compact subset of an unstable manifold $C_{U} \subset W_{U}^{u}(a) \subset A_{U}$.

\subsection{Algorithm.}

Algorithm 2.

1. Let the set of variables be the set of vertices in a graph that we will call $R G$.

2. For every variable $x$, build the reconstructed attractor $A_{x}:=\Phi_{\left(\phi^{T}, \varphi_{x}, k_{x}\right)}(A)$, where $\varphi_{x}$ depends only on $x$.

3. For every pair $x$ and $y$, test, using some criterion, whether there is a surjection

$$
f: \bigcup_{a} W_{1}^{u}(a) \subset A_{x} \rightarrow \bigcup_{b} W_{2}^{u}(b) \subset A_{y}
$$

that is continuous on any $C_{1} \subset W_{1}^{u}(a) \subset A_{x}$, and a surjection

$$
g: \bigcup_{b} W_{2}^{u}(b) \subset A_{y} \rightarrow \bigcup_{a} W_{1}^{u}(a) \subset A_{x}
$$

that is continuous on any $C_{2} \subset W_{2}^{u}(b) \subset A_{y}$, such that if $f$ and $g$ are injective, then $f^{-1}=g$. Here, $W_{1}^{u}(a)$ and $W_{2}^{u}(b)$ are the delay images of the unstable manifolds $W_{U}^{u}(a) \subset A_{U}$ and $W_{V}^{u}(b) \subset A_{V}$ for $x \in\left|U_{\min }\right|, y \in\left|V_{\min }\right|$.

4. If $f$ exists, draw an edge $y \rightarrow x$ in $R G$, and if $g$ exists, draw an edge $x \rightarrow y$.

Theorem 4.8. Let $\phi: \mathbb{R}^{n} \rightarrow \mathbb{R}^{n}$ be a diffeomorphism satisfying the assumptions $\mathrm{A} 1$ and $\mathrm{A} 2$ above, or a flow satisfying $\mathrm{B} 1-\mathrm{B} 3$. Assume that $(A, \phi)$ has a strongly resolved attractor structure. Further, given $x \in\left|U_{\min }\right|$, assume there exists a prevalent observation function $\varphi_{x} \in \mathcal{P}_{U}$ that depends only on observations of $x$. Then the following hold:

1. The graph $R G$ constructed by the algorithm is the transitive closure of $\mathcal{I} \mathcal{G}$, less any self-loops.

2. The graph of strongly connected path components of $R G$ is the transitive closure of the component graph CG.

Proof. This proof follows the proof of Theorem 3.13 very closely with the following substitutions: strongly resolved replaces fully resolved; prevalent replaces generic; compact subsets of unstable manifolds $C_{U} \subset W_{U}^{u}(a) \subset A_{U}$ replace manifolds $M_{U}$; and Theorem 4.6 replaces Theorem 3.11.

Algorithm 2 involves only a slight modification of Algorithm 1. We discuss a specific implementation of this modification in the following section.

Copyright (c) by SIAM. Unauthorized reproduction of this article is prohibited. 
5. Practical detection of projections. The main result of our work is that one-way interactions between the strongly connected components of the interaction graph of a dynamical system are identified with one-way surjections between corresponding reconstructions in a fully resolved manifold filtration or strongly resolved attractor filtration. In this section, we briefly describe our implementation of state space reconstruction, we summarize work by Pecora, Carroll, and Heagy [15] for detecting continuous functions between delay reconstructions of invariant compact manifolds, and then we introduce a modification for compact attractors. Finally, we present several examples using the unmodified version of Pecora, Carroll, and Heagy [15]; that is, we assume the presence of an invariant manifold filtration for the dynamical systems.

5.1. State space reconstruction. Given two equally spaced and equally long time series $x=(x(0), x(\tau), x(2 \tau), \ldots, x(n \tau))$ and $y=(y(0), y(\tau), y(2 \tau), \ldots, y(n \tau))$, our task is to produce $M_{x}$ and $M_{y}$. For each reconstruction we must choose an observation function $\varphi$, an appropriate reconstruction dimension $d$, and an integer index lag $i$, where $T=i \tau$ is the time lag mentioned in the previous sections. In practice we choose $\varphi$ to be the projection function, i.e., $\varphi_{x}(M)=x$ and $\varphi_{y}(M)=y$, and assume that these are generic maps in $\mathcal{G}_{U}$ and $\mathcal{G}_{V}$, respectively, where $U$ and $V$ are the minimal upper sets of $x$ and $y$. To choose integer index lags $i_{x}$ and $i_{y}$, we use as a guide the first zero of the autocorrelation function of the time series $x$ and $y$, respectively. The time lags $T_{x}=i_{x} \tau$ and $T_{y}=i_{y} \tau$ are assumed generic.

A point in each reconstruction is given by

$$
\begin{aligned}
& u(j)=\left(x(j \tau), x\left(\left(j-i_{x}\right) \tau\right), \ldots, x\left(\left(j-(d-1) i_{x}\right) \tau\right)\right), \\
& v(j)=\left(y(j \tau), y\left(\left(j-i_{y}\right) \tau\right), \ldots, y\left(\left(j-(d-1) i_{y}\right) \tau\right)\right),
\end{aligned}
$$

where we will consistently use $u$ to denote a point in the reconstruction $M_{x}$ and $v$ to denote a point in the reconstruction $M_{y}$. The index $j$ uniquely identifies each point on either manifold. We do not require that $j$ be a scalar multiple of $i_{x}$ or $i_{y}$. The reconstruction is built as a sliding window across the full length of both time series, instead of only on the points that are multiples of $i_{x}$ or $i_{y}$. This choice leads to a smoother reconstruction and also makes it easier to identify contemporaneous points in $M_{x}$ and $M_{y}$. In particular, $M_{x}$ has $n+1-(d-1) i_{x}$ points and, similarly, $M_{y}$ has $n+1-(d-1) i_{y}$ points (there are $n+1$ points in each of $x$ and $y)$. If $i_{x}<i_{y}$, then the last $n+1-(d-1) i_{y}$ points of $M_{x}$ are contemporaneous with $M_{y}$, in the sense that the first coordinate of both occurs at the same time. From now on, we will restrict $M_{x}$ (or $M_{y}$ if $i_{y}<i_{x}$ ) to this subset, so that $M_{x}$ and $M_{y}$ have the same number of points $N$ with temporal coincidence in the first coordinate.

There is substantial literature addressing the choice of $d$ and $T$ in state space reconstruction; see, e.g., $[2,3,4,5,6,11,12]$. We have chosen deliberately simple methods to demonstrate the recovery of the transitive closure of CG from reconstructions. In all of our examples we know the dimensionality of the system, and we choose $d$ to be the known dimensionality. Given a set of noisy time series and no a priori knowledge about a dynamical system, we would choose a more general reconstruction method such as the one in Casdagli et al. [4].

5.2. Continuity testing. We want to test for the existence of continuous surjections $f$ : $M_{x} \rightarrow M_{y}$ and $g: M_{y} \rightarrow M_{x}$, which are inverses when injective. Natural candidates for 
these functions are the contemporaneous maps given by $f(u(j))=v(j)$ and $g(v(j))=u(j)$ for $u(j) \in M_{x}, v(j) \in M_{y}$. Given the earlier restriction of $M_{x}$ and $M_{y}$ to contemporaneous points, this map is naturally surjective. If both $f$ and $g$ exist, then $f^{-1}=g$ and there is a bijection between $M_{x}$ and $M_{y}$. If both are continuous, then $M_{x}$ and $M_{y}$ are homeomorphic.

Pecora, Carroll, and Heagy [15] begin with the definition of continuity: if $f$ is continuous at $u(j) \in M_{x}$, then for every $\epsilon>0$ there exists $\delta>0$ such that $\|u-u(j)\|<\delta$ implies $\|f(u)-f(u(j))\|<\epsilon$. They note that it is insufficient to merely find such a $\delta$ for a given $\epsilon$; it's necessary to have a null hypothesis against which the likelihood of finding a $\delta$ can be assessed. They propose a null hypothesis that the points in $M_{y}$ are independently and randomly distributed with respect to the points in $M_{x}$, although they emphasize that other null hypotheses can be used. This gives rise to a schema for accepting or rejecting the continuity of $f$ and $g$.

Given $\epsilon>0$ and $\delta>0$, define punctured open sets in $M_{x}$ and $M_{y}$ :

$$
\begin{aligned}
B_{\delta}(u) & :=\left\{z \in M_{x}, z \neq u:\|z-u\|<\delta\right\}, \\
B_{\epsilon}(f(u)) & :=\left\{z \in M_{y}, z \neq f(u):\|z-f(u)\|<\epsilon\right\} .
\end{aligned}
$$

Pecora, Carroll, and Heagy [15] present the following algorithm:

1. For a fixed $\epsilon$, guess an initial $\delta$. If $f\left(B_{\delta}(u)\right) \subseteq B_{\epsilon}(f(u))$, continue to the next step. Otherwise, reduce $\delta$ and repeat until successful or until $B_{\delta}(u)=\emptyset$. This step ensures that the points of interest are consistent with the definition of continuity.

2. Let $n_{\delta}=\left|B_{\delta}(u)\right|$ be the number of points in the $\delta$-ball, and similarly let $n_{\epsilon}=$ $\left|B_{\epsilon}(f(u))\right|$. The probability under the null hypothesis that $f\left(B_{\delta}(u)\right) \subseteq B_{\epsilon}(f(u))$ is given by $p=\left(n_{\epsilon} / N\right)^{n_{\delta}}$, where $N$ is the number of points in $M_{y}$.

3. If this probability is sufficiently low, the null hypothesis may be rejected at $u$. The pointwise confidence for rejecting the null hypothesis is given by $\Theta(\epsilon, u)=1-\left(p / p_{\max }\right)$, where $p_{\max }$ is the maximum of the binomial distribution

$$
b\left(k ; n_{\delta}, p\right)=\left(\begin{array}{c}
n_{\delta} \\
k
\end{array}\right) p^{k}(1-p)^{n_{\delta}-k}
$$

and the maximum value occurs at $k=$ floor $\left(\left(n_{\delta}+1\right) p\right)$.

4. Calculate a scalar confidence for the whole manifold by averaging the pointwise confidences over a randomly chosen set of $n$ points indexed by the set $I$ :

$$
\Theta_{x y}(\epsilon)=\frac{1}{n} \sum_{j \in I} \Theta(\epsilon, u(j))
$$

Remark 1. In a similar fashion, the confidence in the reverse direction, $\Theta_{y x}(\epsilon)$ for $g: M_{y} \rightarrow$ $M_{x}$, may be calculated. The $(\epsilon, \delta)$ pairs in the $\Theta_{y x}(\epsilon)$ calculation will be entirely independent of the values used in the $\Theta_{x y}(\epsilon)$ calculation.

If $\Theta_{x y}(\epsilon)$ is near 1, then we accept that the points mapping from $\delta$-balls in $M_{x}$ to $\epsilon$-balls in $M_{y}$ are nonrandom events. This constitutes circumstantial evidence for the continuity of $f: M_{x} \rightarrow M_{y}$, since the points were chosen to be consistent with the definition of continuity. Similarly, the continuity of $g$ is supported for high values of $\Theta_{y x}(\epsilon)$.

Copyright ( $\odot$ by SIAM. Unauthorized reproduction of this article is prohibited. 
There is not a clear way to choose an appropriate $\epsilon$ for a given problem. If $\epsilon$ is too small, then $n_{\epsilon}=0$ and continuity will never be observed. But if $\epsilon$ is too large, e.g., half the size of the manifold, then continuity may be spuriously detected. Like Pecora, Carroll, and Heagy [15], we test a number of different $\epsilon$ values nondimensionalized by the standard deviations of the reconstructions. By this we mean the standard deviation of the distance from each point to the mean of the reconstruction in $\mathbb{R}^{d}$. A continuous function should ideally show a monotonic increase in $\Theta_{x y}$ as $\epsilon$ increases, and should show a relatively high $\Theta_{x y}$ for relatively low values of $\epsilon$.

As a time series increases in length, the reconstructed points fill in the invariant compact manifold. If the functions $f$ and $g$ are continuous, then the confidence of continuity should increase as more points are included in the calculation. So we also calculate $\Theta_{x y}$ and $\Theta_{y x}$ using an increasing number of points from the time series $x$ and $y$. That is, we make a family of reconstructions, $M_{x}^{N_{1}}, M_{x}^{N_{2}}, \ldots, M_{x}^{N_{k}}$, where $N_{1}, N_{2}, \ldots, N_{k}$ indicate an increasing number of points in the reconstructions. If the family of $\Theta_{x y}(\epsilon)$ curves converges upward toward 1 with increasing $N_{i}$, then we have strong circumstantial evidence for continuity.

The fact that we draw conclusions based on the contemporaneous functions $f(u(j))=v(j)$ and $g(v(j))=u(j)$ comes from the assumption that the underlying dynamical system does not have an explicit delay. If we suspect that there is a delay in the system, then we should check continuity of the delay maps $f_{k}(u(j))=v(j+k)$ and $g_{k}(v(j))=u(j-k)$ for some $k$. In practice, it is not possible to test all such maps, and in our differential equation examples the contemporaneous maps are sufficient.

5.3. Continuity testing between compact subsets of unstable manifolds. To approximate compact subsets of unstable manifolds in reconstructed attractors $A_{x}$ and $A_{y}$, we use the notation introduced in (9) for $u(j) \in A_{x}$ and contemporaneous point $v(j) \in A_{y}$. The induced dynamics on $A_{x}$ are given by the shift map $F(u(j)):=u(j+1)$ and on $A_{y}$ by $G(v(j)):=v(j+1)$. Take a point $u \in A_{x}$. To approximate a $\delta$-neighborhood of the unstable manifold $W_{x}^{u}(u) \subset A_{x}$, we look at the forward image of neighborhood surrounding a preimage of $u$. Let

$$
U_{\delta}(u):=\left\{z \in A_{x}, z \neq u:\|u-z\|<\delta\right\}
$$

Then

$$
B_{\delta}^{n}(u):=U_{\delta}(u) \cap F^{n}\left(U_{\delta}\left(F^{-n}(u)\right)\right) .
$$

Note that $B_{\delta}^{n}(u)$ approximates a $\delta$-neighborhood on the unstable manifold $W_{x}^{u}(u) \subset A_{x}$ for large $n$. Similarly, we define the corresponding neighborhoods in $W_{y}^{u}(f(u))$

$$
B_{\epsilon}^{n}(f(u)):=U_{\delta}(f(u)) \cap G^{n}\left(U_{\delta}\left(G^{-n}(f(u))\right)\right),
$$

which approximates the $\epsilon$-neighborhood on the unstable manifold $W_{y}^{u}(f(u)) \subset A_{y}$ for large $n$.

We then test continuity of a contemporaneous map $f: A_{x} \rightarrow A_{y}$ on compact subsets of unstable manifolds by fixing moderate to large values of $n$ and then proceed with the previous algorithm restricted to sets $B_{\delta}^{n}(u)$ and $B_{\epsilon}^{n}(f(u))$.

Copyright $\odot$ by SIAM. Unauthorized reproduction of this article is prohibited. 
5.4. Chaotic oscillator example. We begin with a two-tier chaotic oscillator written as a set of first order equations:

$$
\begin{aligned}
\dot{x} & =y, \\
\dot{y} & =\mu\left(1-x^{2}\right) y-x, \\
\dot{z} & =w, \\
\dot{w} & =-w-\beta \sin (z)+A \sin (x) .
\end{aligned}
$$

The interaction and component graphs are given in Figure 9.
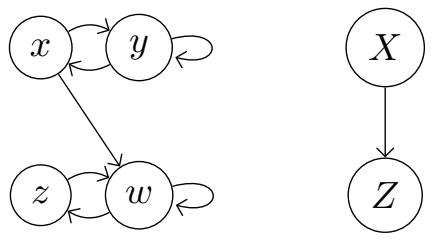

Figure 9. IG and CG for the chaotic oscillator example.

The parameter choices and initial conditions we used are

$$
\begin{aligned}
\mu & =4.0, \quad \beta=1.2, \quad A=2.0, \\
x(0)=1.0, \quad z(0) & =3.0, \quad y(0)=w(0)=2.0 .
\end{aligned}
$$

The equations were solved using the Runge-Kutta fourth order algorithm with a fixed time step of $\tau=0.025$. The result is a chaotic attractor in a bounded region of space; see the phase portraits in Figure 10. There are nearby parameter choices that result in an unbounded attracting invariant set, and state space reconstruction techniques are not guaranteed to work on such attractors. The reconstructions $M_{x}, M_{y}, M_{z}, M_{w}$ were created in $\mathbb{R}^{4}$ with a time lag of $T=100 \tau$ for every variable except $z$, which had a lag of $T_{z}=115 \tau$.

To calculate the continuity confidences, we had to choose a set of time series lengths to build reconstructions, a set of $\epsilon$ values, and sets of random points on which to perform the continuity test. The solution trajectory was simulated out to time 1200 , and a family of reconstructions was created using $30-100 \%$ of the full time series. The continuity test was performed on $10 \%$ of the points in each reconstruction chosen randomly. The points were chosen independently for each reconstruction and each pairwise comparison. The set of $\epsilon$ values sampled the interval between 0.02 and 0.4 standard deviations of the full length reconstruction for each variable. We did not recalculate the standard deviation for reconstructions of shorter lengths.

The component graph CG was recovered as expected. In particular, the homeomorphisms $M_{x} \leftrightarrow M_{y}$ and $M_{z} \leftrightarrow M_{w}$ were confirmed, and one-way continuous surjections were seen going from $M_{z}, M_{w}$ to $M_{x}, M_{y}$. The confidence graphs for continuous $M_{x} \rightarrow M_{w}$ and $M_{w} \rightarrow M_{x}$ are shown as illustrative examples in Figure 11. A set of $\Theta(\epsilon)$ curves is plotted, where the curves are parameterized by the length of the reconstruction. The legend shows the proportion of points in the time series used to make the reconstructions. The direction $M_{x} \rightarrow M_{w}$ is associated with a one-way interaction from $w$ to $x$. The confidence values are 

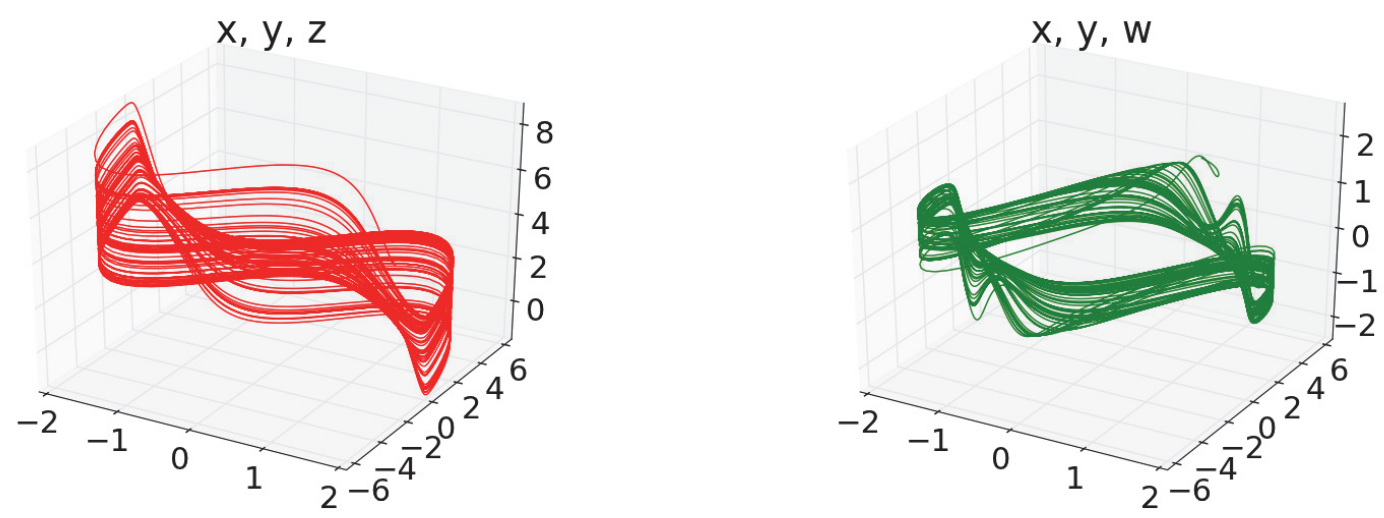

Figure 10. Three-dimensional phase spaces for the chaotic oscillator example.
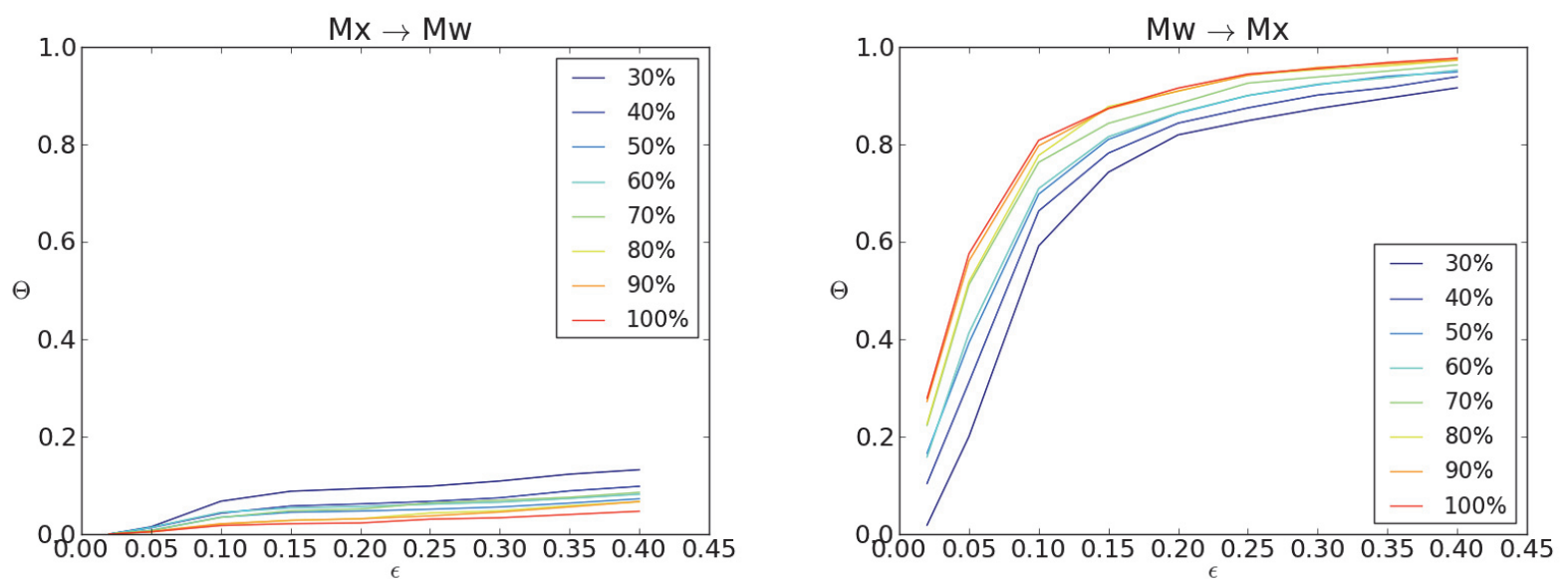

Figure 11. Chaotic oscillator example. Families of curves of $\Theta_{x w}$ and $\Theta_{w x}$ as functions of $\epsilon$ scaled by the standard deviation of $M_{w}$ and $M_{x}$, respectively. The family of curves is parameterized by the proportion of points used to make the reconstructions. $\Theta_{w x}$ converges upward toward 1 with increasing $\epsilon$ and increasing reconstruction length, which is evidence for continuity and an edge $x \rightarrow w$. $\Theta_{x w}$ decreases with increasing reconstruction length and has low confidence, so we conclude $w \nrightarrow \rightarrow x$.

low $(<0.2)$ over the range of tested $\epsilon$ values. Moreover, as the reconstruction becomes longer, the confidence decreases. So we conclude correctly that $w$ is not a dynamical driver of $x$. In the other direction, $M_{w} \rightarrow M_{x}$, we see a robust upward convergence with increasing $\epsilon$ and reconstruction length, indicating strong evidence for a continuous function. We conclude that $x$ is a driver of $w$ and draw an edge from $x$ to $w$ in the interaction graph.

We summarize all the variable relationships in Table 1. In the left table, we give the highest confidence value for each pairwise comparison with $\epsilon$ at 0.4 standard deviations and with full length reconstructions. In the right table, we say whether the family of $\Theta(\epsilon)$ curves converged upward or downward or was coincident as the reconstruction length increased. If 
Table 1

Chaotic oscillator example. Left: Confidence values from row reconstruction to column reconstruction when $\epsilon$ is 0.4 standard deviations of the column reconstruction and $100 \%$ of the time series is used in the reconstruction. Right: The behavior of the family of $\Theta(\epsilon)$ curves with increasing reconstruction length: upward convergence (u), downward convergence (d), or coincident curves (c).

\begin{tabular}{c|cccc}
\hline & $M_{x}$ & $M_{y}$ & $M_{z}$ & $M_{w}$ \\
\hline$M_{x}$ & $\cdot$ & 1.00 & 0.04 & 0.05 \\
$M_{y}$ & 1.00 & $\cdot$ & 0.03 & 0.04 \\
$M_{z}$ & 0.95 & 0.98 & $\cdot$ & 1.00 \\
$M_{w}$ & 0.98 & 0.99 & 0.99 & $\cdot$ \\
\hline
\end{tabular}

\begin{tabular}{c|cccc}
\hline & $M_{x}$ & $M_{y}$ & $M_{z}$ & $M_{w}$ \\
\hline$M_{x}$ & $\cdot$ & $\mathrm{c}$ & $\mathrm{d}$ & $\mathrm{d}$ \\
$M_{y}$ & $\mathrm{c}$ & $\cdot$ & $\mathrm{d}$ & $\mathrm{d}$ \\
$M_{z}$ & $\mathrm{u}$ & $\mathrm{u}$ & $\cdot$ & $\mathrm{u}$ \\
$M_{w}$ & $\mathrm{u}$ & $\mathrm{u}$ & $\mathrm{u}$ & $\cdot$ \\
\hline
\end{tabular}
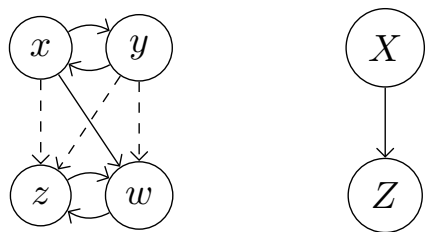

Figure 12. Chaotic oscillator example. Recovered graphs $\overline{\mathcal{I} G}$ and $\overline{C G}$ from continuity testing. Dashed edges are not present in the original interaction graph in Figure 9.

there are high confidence values and anything but downward convergence, we conclude the presence of a continuous surjection and an appropriate edge in the reconstructed interaction graph. With downward convergence or low confidence values, we conclude the absence of an edge. The reconstructed graphs are given in Figure 12.

5.5. Diamond graph example. The point of this example is to explore a more complex component graph shaped like a diamond. We add a second branch to the chaotic oscillator example consisting of a Rössler system driven by the periodic oscillator. Both the chaotic oscillator and the Rössler systems feed into the evolution of an additional variable. The system of equations for the diamond example are

$$
\begin{aligned}
\dot{x} & =y, \\
\dot{y} & =\mu\left(1-x^{2}\right) y-x, \\
\dot{z} & =w, \\
\dot{w} & =-w-\beta \sin (z)+A \sin (x), \\
\dot{s} & =-u-v, \\
\dot{u} & =s+a u, \\
\dot{v} & =b+v\left(\frac{2+C(x-3)}{2} s-c\right)-\frac{C(x-3)}{4}\left(s^{2}+v^{2}\right), \\
\dot{p} & =-p+B \sin (v) \sin (z) .
\end{aligned}
$$

When the parameter $C$ is 0 , the equations for $s, u, v$ reduce to the usual Rössler system. When $C$ is nonzero, there is an additional nonlinear term not present in the original equations. The interaction and component graphs for the whole system are given in Figure 13.

Copyright ( $\odot$ by SIAM. Unauthorized reproduction of this article is prohibited. 

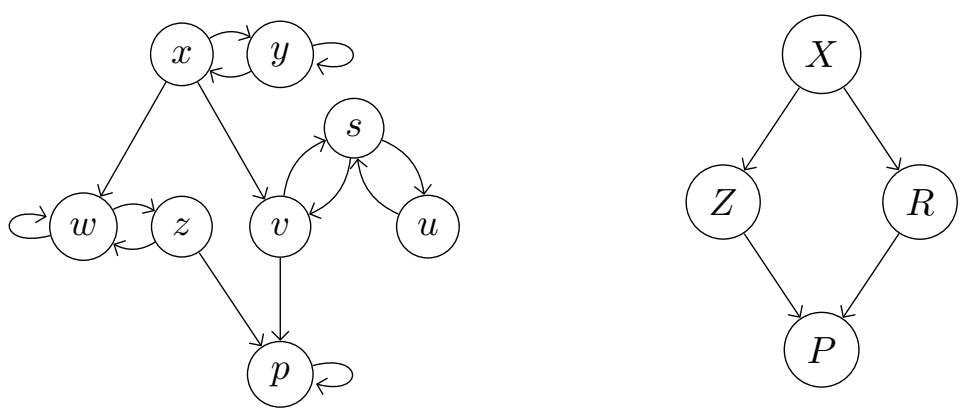

Figure 13. IG and CG for the diamond example.

The parameter choices and initial conditions we used are

$$
\begin{array}{r}
\mu=4.0, \quad \beta=1.2, \quad A=2.0, \quad B=1.25, \quad C=0.20, \quad a=0.2, \quad b=0.2, \quad c=5.7, \\
x(0)=1.0, \quad y(0)=w(0)=2.0, \quad z(0)=3.0, \quad s(0)=u(0)=0.5, \quad v(0)=0.1, \quad p(0)=0.75 .
\end{array}
$$

All of the chaotic oscillator parameters and numerical parameters are the same as in the previous example, except that the reconstructions were created in $\mathbb{R}^{8}$ with a time lag of $T=95 \tau$ for $M_{w}, T=60 \tau$ for $M_{s}, T=65 \tau$ for $M_{u}, T=50 \tau$ for $M_{v}$, and $T=100 \tau$ for $M_{p}$.

Continuity testing was performed in the same manner as before, and the transitive closures of the graphs in Figure 13 were recovered. We show example confidence graphs in Figure 14. When testing $M_{p} \rightarrow M_{v}$, the curves $\Theta_{p v}(\epsilon)$ coincide very closely and converge toward 1,

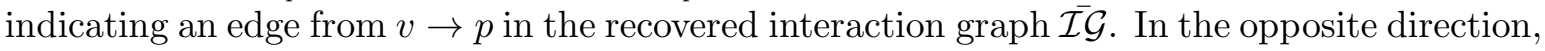
$\Theta_{v p}(\epsilon)$ attains moderately high confidence at short reconstruction lengths, but the confidence converges downward as the reconstruction length increases. Thus we conclude that there is not a reciprocal edge from $p$ to $v$. The graph of confidences for $M_{s} \rightarrow M_{x}$ has only moderate values, but $\Theta_{s x}(\epsilon)$ converges robustly upward with reconstruction length. We therefore conclude there is an edge from $x$ to $s$.

Summary data for all pairwise comparisons are listed in Table 2. The recovered graphs constructed from the confidence values and convergence trends are shown in Figure 15, where dashed lines denote connections not found in the original graphs.

5.6. Measurement noise. It is obvious that sufficiently high noise can overwhelm a deterministic signal. In this section, we characterize how much measurement noise is required to interfere with the recovery of the transitive closure of the component graph of the chaotic oscillator example. See section 5.4 for the equations and parameters.

By measurement noise we mean noise that is introduced when making observations of the manifold $M$, so that the result is a perturbation of the observation function: $\varphi+\xi(t)$. The noise function $\xi(t)$ is a sequence of realizations of a probability distribution $\mathcal{N}(\mu, \sigma)$ with mean $\mu$ and standard deviation $\sigma$, often taken to be the normal distribution. Measurement noise is distinct from stochastic elements in the dynamical system (dynamic noise) and from estimation errors that arise from using a finite length time series in the reconstruction [4].

Let $\sigma_{x}, \sigma_{y}, \sigma_{z}$, and $\sigma_{w}$ denote the standard deviations of the deterministic time series $x, y$, $z$, and $w$. We perturb the time series with independent realizations $\xi_{x}(t), \xi_{y}(t), \xi_{z}(t), \xi_{w}(t)$, 

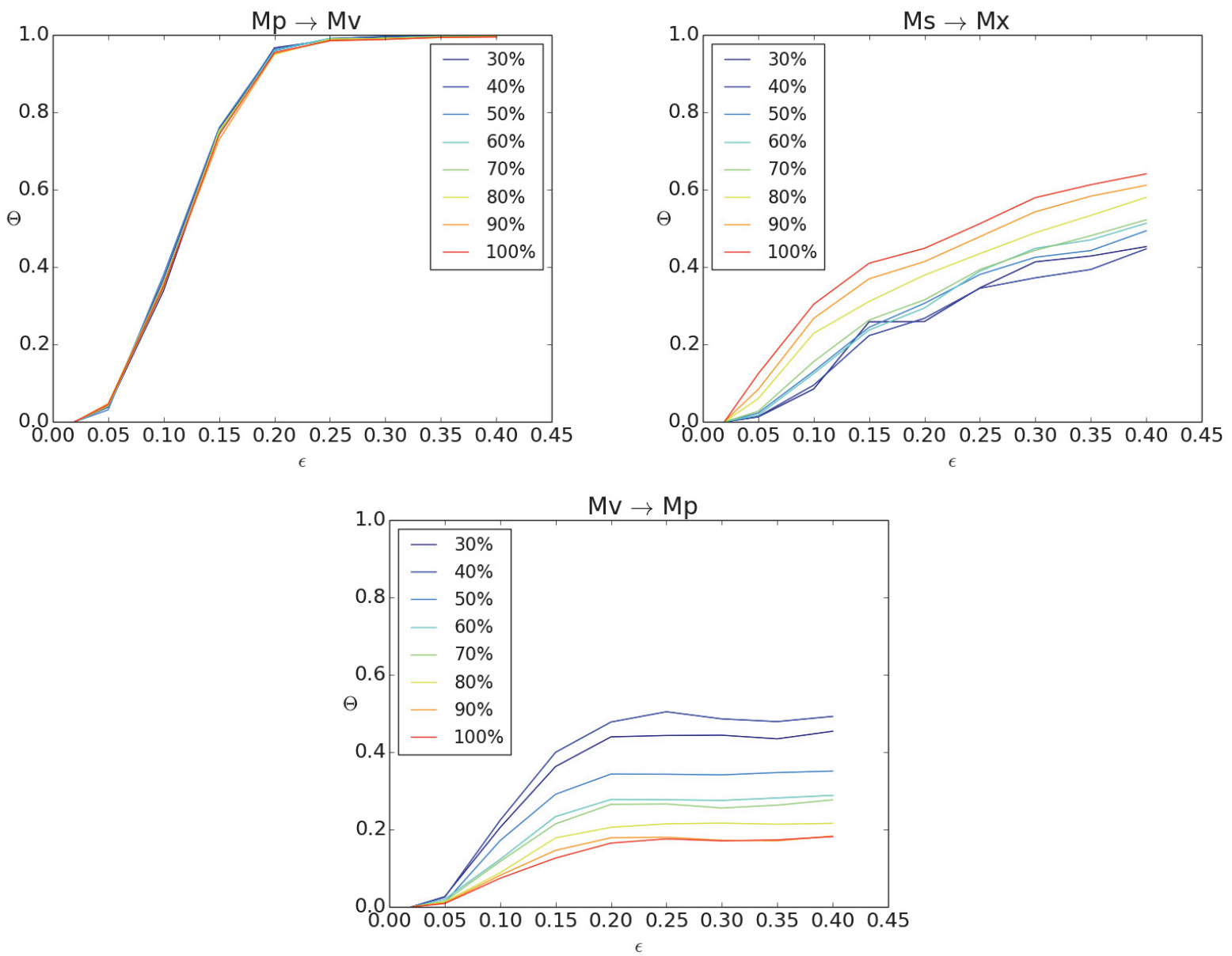

Figure 14. Diamond example. Confidences $\Theta_{p v}, \Theta_{s x}$, and $\Theta_{v p}$ vs $\epsilon$ scaled by the standard deviation of the ranges $\left(M_{v}, M_{x}\right.$, and $\left.M_{p}\right)$. The legend indicates the percentage of points used to make the reconstructions. The coincident set of $\Theta_{p v}$ curves converges upward toward 1 with increasing $\epsilon$, implying continuity and an edge $v \rightarrow p . \Theta_{s x}$ shows the same robust upward trend with lower, but still acceptable, confidence levels that support $x \rightarrow s . \Theta_{v p}$ decreases with increasing reconstruction length, implying $p \not \rightarrow v$, even though the confidence levels are moderate.

according to the normal distributions $\mathcal{N}\left(0, q \sigma_{x}\right), \mathcal{N}\left(0, q \sigma_{y}\right), \mathcal{N}\left(0, q \sigma_{z}\right), \mathcal{N}\left(0, q \sigma_{w}\right)$, where the parameter $q$ increases from 0.01 to 0.1 . That is, we add noise with $1 \%$ to $10 \%$ of the standard deviation of the time series, and perform continuity testing precisely as before on the perturbed series $\hat{x}=x(t)+\xi_{x}(t), \hat{y}=y(t)+\xi_{y}(t)$, etc.

In Figure 16, we show the decrease in continuity confidence that occurs with increasing noise. The noise levels are given in the legend as the percentage of the time series standard deviation. All reconstructions used $100 \%$ of the time series (up to time 1200 as before). The confidence in the $M_{x} \rightarrow M_{y}$ direction, $\Theta_{x y}(\epsilon)$, is fairly robust to increasing noise of this magnitude, indicating that the edge $y \rightarrow x$ in $\mathcal{I} \mathcal{G}$ will be consistently recovered. However, $\Theta_{y x}(\epsilon)$ decays rapidly, and so the edge from $x \rightarrow y$ is lost with noise at $3-4 \%$ of the standard deviation. The two graphs in Figure 16 exemplify the patterns seen in all pairwise comparisons

Copyright (C) by SIAM. Unauthorized reproduction of this article is prohibited. 
Table 2

Diamond example. Pairs of confidence values and convergence trends from row to column reconstructions. Confidence values are taken when $\epsilon$ is 0.4 the standard deviation of the column reconstruction and $100 \%$ of the time series is used in the reconstruction. Convergence trends in $\Theta(\epsilon)$ with increasing reconstruction length are denoted as u (upward convergence), d (downward convergence), or c (coincident curves). Values for the chaotic oscillator have been elided.

\begin{tabular}{c|cccccccc}
\hline & $M_{x}$ & $M_{y}$ & $M_{z}$ & $M_{w}$ & $M_{s}$ & $M_{u}$ & $M_{v}$ & $M_{p}$ \\
\hline$M_{x}$ & $\cdot$ & $\cdot$ & $\cdot$ & $\cdot$ & $(0.00, \mathrm{c})$ & $(0.00, \mathrm{c})$ & $(0.00, \mathrm{c})$ & $(0.00, \mathrm{c})$ \\
$M_{y}$ & $\cdot$ & $\cdot$ & $\cdot$ & $\cdot$ & $(0.00, \mathrm{c})$ & $(0.00, \mathrm{c})$ & $(0.00, \mathrm{c})$ & $(0.00, \mathrm{c})$ \\
$M_{z}$ & $\cdot$ & $\cdot$ & $\cdot$ & $\cdot$ & $(0.28, \mathrm{~d})$ & $(0.28, \mathrm{~d})$ & $(0.32, \mathrm{~d})$ & $(0.29, \mathrm{~d})$ \\
$M_{w}$ & $\cdot$ & $\cdot$ & $\cdot$ & $\cdot$ & $(0.17, \mathrm{~d})$ & $(0.18, \mathrm{~d})$ & $(0.21, \mathrm{~d})$ & $(0.17, \mathrm{~d})$ \\
$M_{s}$ & $(0.64, \mathrm{u})$ & $(0.74, \mathrm{u})$ & $(0.06, \mathrm{~d})$ & $(0.08, \mathrm{~d})$ & $\cdot$ & $(0.99, \mathrm{u})$ & $(0.99, \mathrm{u})$ & $(0.09, \mathrm{~d})$ \\
$M_{u}$ & $(0.64, \mathrm{u})$ & $(0.78, \mathrm{u})$ & $(0.07, \mathrm{~d})$ & $(0.10, \mathrm{~d})$ & $(1.00, \mathrm{u})$ & $\cdot$ & $(1.00, \mathrm{u})$ & $(0.09, \mathrm{~d})$ \\
$M_{v}$ & $(0.69, \mathrm{u})$ & $(0.81, \mathrm{u})$ & $(0.17, \mathrm{~d})$ & $(0.17, \mathrm{~d})$ & $(0.98, \mathrm{u})$ & $(0.97, \mathrm{u})$ & $\cdot$ & $(0.18, \mathrm{~d})$ \\
$M_{p}$ & $(0.85, \mathrm{c})$ & $(0.99, \mathrm{c})$ & $(0.98, \mathrm{c})$ & $(0.99, \mathrm{c})$ & $(0.99, \mathrm{c})$ & $(0.99, \mathrm{c})$ & $(0.99, \mathrm{c})$ & $\cdot$ \\
\hline
\end{tabular}
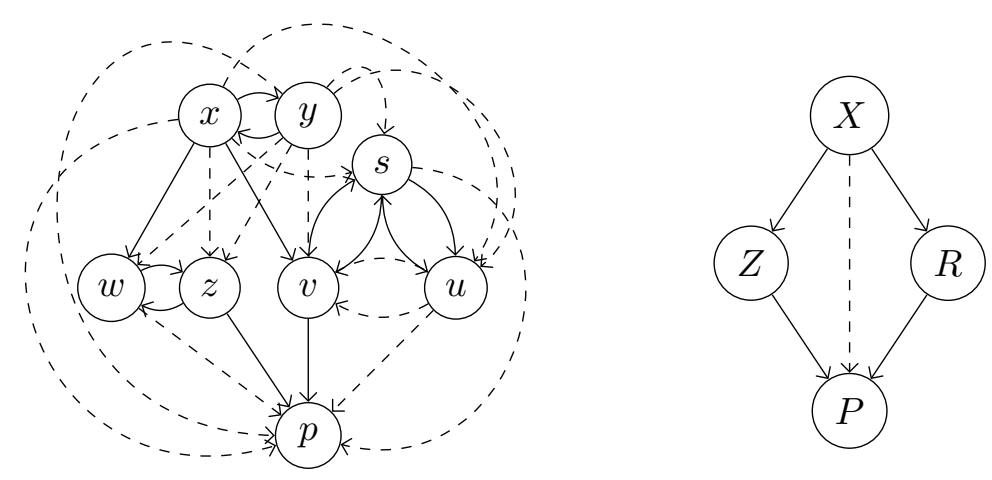

Figure 15. Recovered graphs $\overline{\mathcal{I} G}$ and $\overline{C G}$ for the diamond example.

between dynamically related variables. The confidences $\Theta_{w y}$ and $\Theta_{z y}$ are very similar to $\Theta_{x y}$; on the other hand, $\Theta_{w x}, \Theta_{z x}, \Theta_{w z}$, and $\Theta_{z w}$ are substantially like $\Theta_{y x}$. All other pairwise comparisons originally failed the continuity test, and they changed very little with the application of noise. We conclude that the correct reconstruction of the dynamic interactions in the chaotic oscillator example can be recovered with measurement noise in each variable up to $2-3 \%$ of the standard deviation.

6. Discussion. In this contribution we describe mathematical structures that underlie a causality detection method based on delay reconstructions from individual time series introduced by Sugihara et al. [19]. We discuss these issues both in Takens' framework of manifold reconstruction as well as in the Sauer, Yorke, and Casdagli [16] framework of attractor reconstruction. In any dynamical system we define an interaction graph that summarizes the forcing relationship between variables: if variable $x$ occurs on the right-hand side of the evolution of variable $y$, then we call $x$ a dynamical driver of $y$ and a vertex $x$ is connected by

Copyright (C) by SIAM. Unauthorized reproduction of this article is prohibited. 

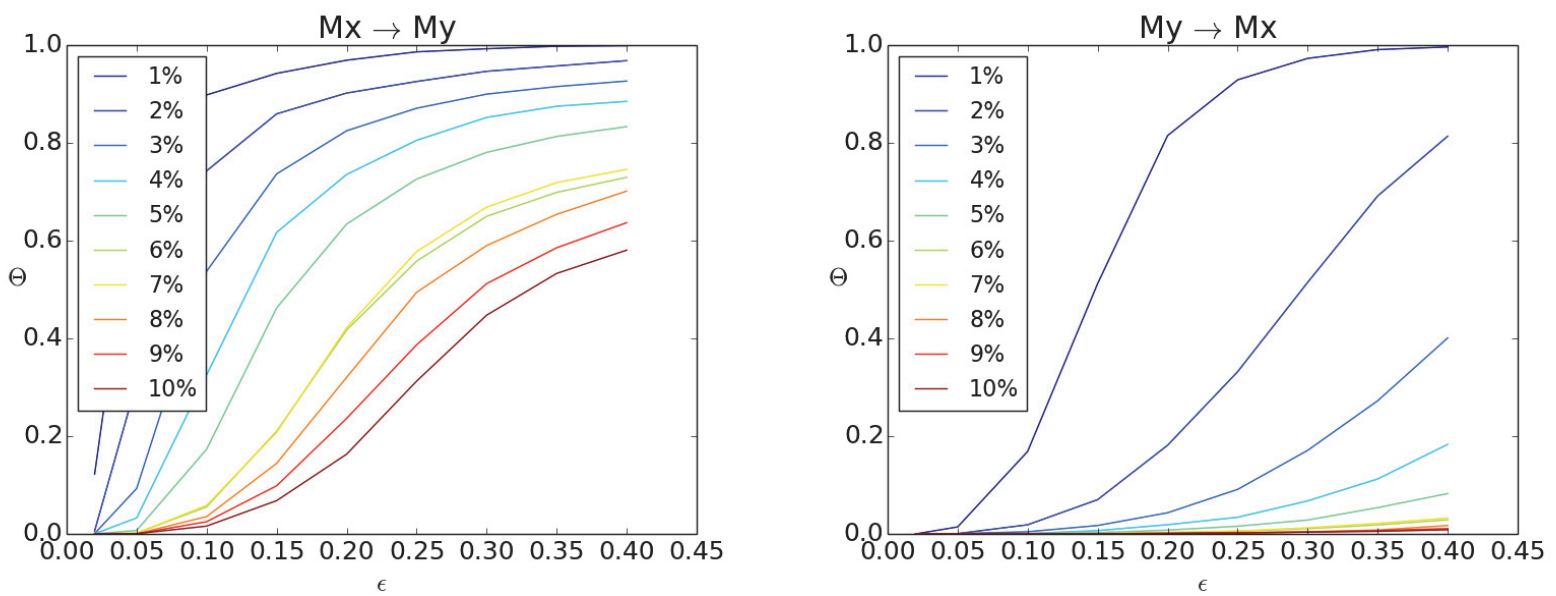

Figure 16. Continuity testing for $M_{x} \rightarrow M_{y}$ and $M_{y} \rightarrow M_{x}$ with increasing noise as indicated in the legend. $\Theta_{x y}$ is more strongly preserved under perturbation.

a directed edge to a vertex $y$. We show that strongly connected path components of the interaction graphs are the finest units that any method based on delayed reconstruction will be able to recover. In fact the best one can hope for is a transitive closure of the component graph, i.e., the induced graph on the connected path components.

There is a slight shift of emphasis between the approaches of Sugihara et al. [19] and Takens [20]. In Takens' results the observation function is arbitrary, and the emphasis is on the fact that we can change a nongeneric observation function slightly to get a generic one. In Sugihara et al. [19], as well as in the applications of time series reconstruction in general, the observation functions are fixed experimental data series and thus cannot be changed. One hopes, based on Takens' genericity results, that these fixed observation functions belong to the generic set. However, this is an assumption which cannot be verified by perturbing the observation function slightly and checking if the results remain the same.

Given a set of time series measurements, we assume that there is an underlying system of differential equations and an associated interaction graph. Each edge in the reconstructed interaction graph represents a relationship that is potentially causal in the physical system. The driving variable may be a direct cause of the driven variable, an indirect cause, a correlate of an indirect or direct cause, or an artifact from a lack of full resolution in the dynamical system. This is why we emphasize that the relationships we find are only hypotheses for causal inference. Each specific case will require independent examination to decide if a driving relationship is also a causal relationship. Similarly, one can hypothesize which variables are not causally related, due to the absence of a continuous surjection between manifolds (or subsets of attractors). Again, this is only a hypothesis, since excessive noise could be masking an underlying relationship, or there could be symmetry in the system.

Sugihara et al. [19] noted that their method based on delay reconstructions works for moderately coupled subsystems. If the coupling is too weak, we cannot detect the effect of one system (or variable) on the other; if the coupling is too strong, then the driving system and driven system synchronize and the reconstructed attractors are identical. We formalize this

Copyright ( $)$ by SIAM. Unauthorized reproduction of this article is prohibited. 
observation with the notion of a fully resolved manifold (attractor) structure, which says that the manifolds parameterized by the upper sets of component graph must be distinguishable. We show that such a fully resolved structure can be recovered by methods based on delay embeddings, up to the resolution of the transitive closure of the component graph.

We further illustrate this point in Figure 17. Graph (a) is the component graph associated with the example in Figure 4 used throughout the paper, and graph (b) is the recovered component graph when the example has a fully resolved manifold structure and Algorithm 1 works perfectly. The reconstructed component graph $\overline{C G}$ has additional connections from $T$ to $Q$ and $P$, shown as dashed lines. Using delay reconstruction techniques on a purely deterministic system, we cannot know if a variable in $T$ directly drives a variable in $Q$, or whether there is only indirect dynamical driving. This is a limitation of using state space reconstructions to recover deterministic dynamical interactions.

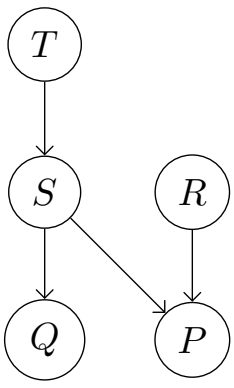

(a)

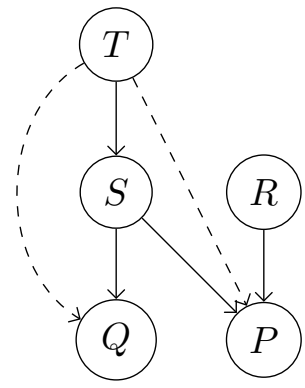

(b)

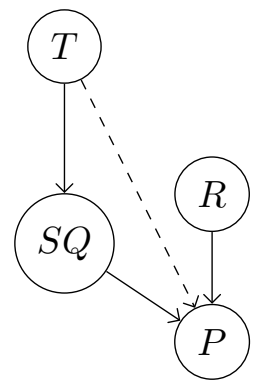

(c)

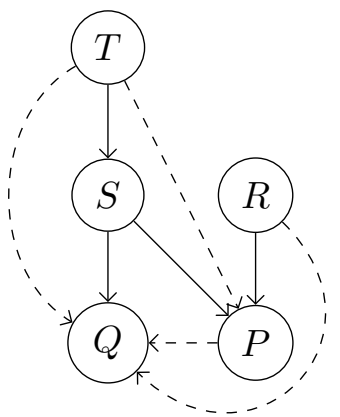

(d)

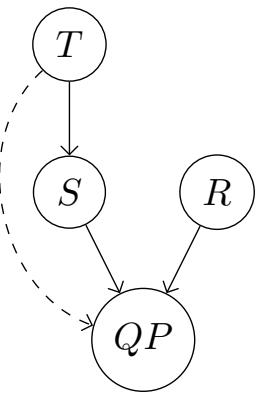

(e)

Figure 17. (a) Example component graph $\mathrm{CG}_{\phi}$ from Figure 4; (b) the transitive closure recovered from a perfect performance of Algorithm 1 when $\mathbb{M}$ is fully resolved; (c) the graph minor when $M_{\{T S Q\}}$ and $M_{\{T S\}}$ are homeomorphic; (d) the graph when there is a continuous surjection from $M_{\{T S Q\}}$ to $M_{\{T R S P\}}$; and (e) the graph when $M_{\{T S Q\}}$ and $M_{\{T R S P\}}$ are homeomorphic. Dashed lines indicate connections that are recovered by the algorithm that are not in $\mathrm{CG}_{\phi}$.

If the descending filtration $\mathbb{M}$ is not fully resolved, then the recovered graph CG may have false edges or collapsed nodes. If condition 1 of the definition fails, then one of the projections in $\mathbb{M}$ is injective and two comparable manifolds cannot be distinguished. If condition 2 fails, then two noncomparable elements of the filtration are connected, and erroneously identified if the condition fails in both directions. These three cases are given in Figure 17. In graph (c), the projection from $M_{\{T S Q\}}$ to $M_{\{T S\}}$ is injective, so that the reconstructed component graph CG is a graph minor that combines nodes $S$ and $Q$. In graph (d), the contemporaneous map described in section 5 is a continuous surjection from $M_{\{T S Q\}}$ to $M_{\{T R S P\}}$, leading to a false edge between $P$ and $Q$, and also between $R$ and $Q$. The latter edge occurs by composing the contemporaneous maps $M_{\{T S Q\}} \rightarrow M_{\{T R S P\}} \rightarrow M_{\{R\}}$. If the inverse map exists and is continuous, then $P$ and $Q$ will be falsely identified as part of the same strongly connected component $P \cup Q$ as in graph (e) of Figure 17.

The failure of condition 1 in the definition of fully resolved is less severe than the failure of condition 2, since the former results in a graph minor of the transitive closure of CG, while 
the latter creates spurious edges not supported by direct or indirect driving relationships. A fully resolved filtration is crucial for the maximal recovery of the true dynamics.

To recover the information about dynamical drivers we use the method of Pecora, Carroll, and Heagy [15], which utilizes a hypothesis-testing framework to detect continuous maps. This is different from the convergent cross-mapping technique proposed in Sugihara et al. [19], which emphasizes the predictability of one time series from another. One key feature of the convergent cross-mapping technique is a projection back to a scalar time series after making the state space reconstructions. We believe that it is more consistent with the theory to test relationships between the state space reconstructions directly, as the method of Pecora, Carroll, and Heagy [15] does. However, we concur with Sugihara et al. [19] that the critical factor supporting the existence of a driving interaction is convergence to an appropriate asymptotic value with increasing time series length, rather than only depending on the results for a single time series length.

Detection of causal relationships between variables represented by experimental time series is an extremely important challenge for present day science. As the amount of data that we are able to collect expands rapidly, our ability to analyze that data is lagging behind. Our contribution, which analyzes the advantages and limitations of a class of methods based on delay reconstructions, hopes to narrow the gap between experimental successes and our ability to draw conclusions from the data.

Appendix A. Proofs for section 3. We wish to apply the theorems of Takens to the diffeomorphisms or flows that admit a fixed component graph CG. In order to do this, we wish to establish the genericity of A1 and A2 for diffeomorphisms and B1 and B2 for flows that are restricted to a fixed CG. We consider only the second case here. We first offer a sketch of the proof of the genericity of conditions B1 and B2 for flows and time lags in $C^{2}(M, M) \times \mathbb{R}^{+}$ (Theorem A.1). The proof is very similar to the proof for the genericity of conditions A1 and $\mathrm{A} 2$ in the space of diffeomorphisms $D^{2}(M, M)$. Assuming this latter fact to be true, we then offer the sketch of the proof of Theorem 3.3, which depends on Takens' Theorem 3.1. Afterward, we proceed to the novel result that conditions B1 and B2 are generic in the product space $\mathcal{P}_{\mathrm{CG}} \times \mathbb{R}^{+}$, referring back to Definition 3.5.

In the rest of the appendix, we have found it convenient to distinguish flows from diffeomorphisms using the notation $\psi$ for flows and $\phi$ for diffeomorphisms. Thus a flow $\psi^{t}$ induces a diffeomorphism $\phi^{T}$ for a choice of time lag $T$.

Theorem A.1. Let $X$ be a $C^{2}$ vector field with associated flow $\psi^{t}$ on a compact manifold M. For $T \in \mathbb{R}^{+}$, define the diffeomorphism $\phi^{T}$ as above. Then for fixed $n \in \mathbb{N}$, a subset of elements $\left(\psi^{t}, T\right)$ that satisfies the following properties is open and dense in $C^{2}(M, M) \times \mathbb{R}^{+}$:

B1 $\psi^{t}$ has no periodic orbits of period $k T$ for $k \leq n$.

B2 If $X(x)=0$, then the eigenvalues of the derivative of $\phi^{T}$ evaluated at $x$ are distinct and the moduli are all different from 1.

Proof. The genericity of B1 and B2 follows from the Kupka-Smale theorem for flows [14]. We will briefly sketch the proof here.

Genericity of B1. The Kupka-Smale theorem for flows [14] states that for a residual set of flows in $C^{2}(M, M)$, all of the fixed points and periodic orbits of $\psi^{t}$ are hyperbolic. The Hartman-Grobman theorem for flows [14] states that there exists an open neighborhood $U_{\ell}$ of a 
hyperbolic fixed point or periodic orbit such that the flow in the neighborhood is topologically equivalent to the linearization of the flow at the invariant set. This implies that in each $U_{\ell}$ there is a unique invariant set; i.e., the fixed points and periodic orbits of $\psi^{t}$ are isolated. To see that this implies finiteness of the set of fixed points and periodic orbits on a compact manifold $M$, consider open sets $V_{\ell} \subset U_{\ell}$ such that $C l\left(V_{\ell}\right) \subset U_{\ell}$ and the invariant set is fully contained in $V_{\ell}$. This is possible since the manifold is locally homeomorphic to $\mathbb{R}^{n}$. Then consider the collection of open sets $\left\{U_{\ell}\right\}_{\ell} \cup\left(M \backslash C l\left(\bigcup_{\ell} V_{\ell}\right)\right)$. Since $V_{\ell}$ are disjoint, $C l\left(\bigcup_{\ell} V_{\ell}\right) \subset \bigcup_{\ell} U_{\ell}$ and therefore $M \backslash\left(\bigcup_{\ell} U_{\ell}\right) \subset\left(M \backslash C l\left(\bigcup_{\ell} V_{\ell}\right)\right)$. Therefore this collection is an open cover of a compact manifold $M$ and thus admits a finite subcover. Since every set in the cover contains at most one fixed point or periodic orbit, the number of equilibria and periodic orbits is finite. We have just shown that on a compact manifold the set of flows with a finite number of hyperbolic equilibria and periodic orbits is residual and therefore dense. It is clear that this set is also open in $C^{2}(M, M)$. We denote this set by $G$.

For a given $\psi^{t} \in G$, let $N<\infty$ be the number of hyperbolic periodic orbits and let $T_{1}, T_{2}, \ldots, T_{N}$ denote the minimal periods of each. Fix $n \in \mathbb{N}$ and consider the sets $S_{i}=$ $\left\{T_{i} / k\right\}_{k=1}^{n}$. Each set of real positive numbers $S_{i}$ is finite, so $\mathbb{R}^{+} \backslash \bigcup_{i=1}^{N} S_{i}$ is open and dense in $\mathbb{R}^{+}$. Thus a generic choice of $T \in \mathbb{R}^{+}$, which depends on $\psi^{t}$, ensures that $\{k T\}_{k=1}^{n} \cap$ $\left\{T_{1}, T_{2}, \ldots, T_{N}\right\}=\emptyset$, fulfilling B1. Denote each of these sets by $H_{\psi^{t}}=\mathbb{R}^{+} \backslash \bigcup_{i=1}^{N} S_{i}$.

We know that B1 is fulfilled on the set

$$
\mathbb{G}:=\bigcup_{\psi^{t} \in G}\left\{\psi^{t} \times H_{\psi^{t}}\right\}
$$

which we wish to show is open and dense in $C^{2}(M, M) \times R^{+}$.

To show the density of $\mathbb{G}$, assume that $\left(\psi^{t}, T\right) \notin \mathbb{G}$. Because $G$ is dense in $C^{2}(M, M)$ and $C^{2}(M, M)$ is a metric space, there exists a sequence $\left\{\psi_{i}^{t}\right\} \in G$ with $\psi^{t}$ as its limit point. Then because each $H_{\psi_{i}^{t}}$ is dense in $\mathbb{R}^{+}$, we can choose a sequence $\left\{T_{i}\right\}$ with each $T_{i} \in H_{\psi_{i}^{t}}$ such that $\left|T_{i}-T\right|<1 / i$. Then $\left\{\left(\psi_{i}^{t}, T_{i}\right)\right\} \rightarrow\left(\psi^{t}, T\right)$ shows that every point in $C^{2}(M, M) \times \mathbb{R}^{+}$is the limit point of a sequence in $\mathbb{G}$.

To show openness, let $\left(\psi^{t}, T\right) \in \mathbb{G}$ and let $T_{1}\left(\psi^{t}\right), T_{2}\left(\psi^{t}\right), \ldots, T_{N}\left(\psi^{t}\right)$ be the periods of the hyperbolic orbits of $\psi^{t}$. Because periodic behavior continuously varies in $C^{2}(M, M)$, for any $\delta>0$ there exists an open neighborhood $U_{\delta} \subset G$ of $\psi^{t}$ where $\alpha^{t} \in U_{\delta}$ has the same number of periodic orbits as $\psi^{t}$ and $\left|T_{i}\left(\psi^{t}\right)-T_{i}\left(\alpha^{t}\right)\right|<\delta$ for all $i=1, \ldots, N$. Choose $\delta>0$ small enough so that $\min _{i, k}\left|T-T_{i}\left(\psi^{t}\right) / k\right|>2 \delta$ with $i=1, \ldots, N$ and $k=1, \ldots, n$. This must be possible since $T \in H_{\psi^{t}}$. Now consider a point $\left(\alpha^{t}, \tau\right)$ in the open set $U_{\delta} \times(T-\delta / 2, T+\delta / 2)$. We show that $\tau \in H_{\alpha^{t}}$ by the following argument:

$$
\begin{aligned}
\left|\tau-T_{i}\left(\alpha^{t}\right) / k\right| & \geq|| T-T_{i}\left(\psi^{t}\right) / k|-| T-\tau||-\left|T_{i}\left(\psi^{t}\right) / k-T_{i}\left(\alpha^{t}\right) / k\right| \\
& >3 \delta / 2-\delta / k \\
& \geq \delta / 2 .
\end{aligned}
$$

Thus, $U_{\delta} \times(T-\delta / 2, T+\delta / 2) \subset \mathbb{G}$, so that $\mathbb{G}$ is open in $C^{2}(M, M) \times \mathbb{R}^{+}$.

Genericity of B2. By the Kupka-Smale theorem [14] for an open and dense set of $C^{2}$ vector fields on $M$ the singularities $X(x)=0$ are hyperbolic, and so $D X(x)$ at each singularity has 
no eigenvalues on the imaginary axis. The linearized flow in a small neighborhood about $x$ is given by $\psi^{t}(y)=e^{t D X(x)}(y-x)$, so that the derivative of the diffeomorphism $\phi^{T}$ at $x$ is $e^{T D X(x)}$. Since $T \in \mathbb{R}$, the operator $T D X(x)$ has no eigenvalues on the imaginary axis, implying that $e^{T D X(x)}$ has no eigenvalues on the unit circle.

We now need to prove that the eigenvalues of $e^{T D X(x)}$ are distinct. The proof requires three steps. The first is an observation that there is an open and dense set $\mathcal{A}$ in the set of all linear operators such that $A \in \mathcal{A}$ satisfies two conditions:

1. If $\lambda_{1}$ is an eigenvalue of $A$, then $\operatorname{Re}\left(\lambda_{1}\right) \neq 0$.

2. For any pair of distinct eigenvalues $\lambda_{1}, \lambda_{2}$ of $A$, then $e^{T \lambda_{1}} \neq e^{T \lambda_{2}}$.

It follows that at each singularity $x$, there is an arbitrarily small perturbation that takes $D X(x)$ into a linear operator in $\mathcal{A}$. The second step is to show that the perturbation can be localized around the singularity. While a construction using a bump function is standard (see, e.g., proofs in [14] and [8]), the fact that we need the perturbed function to be $C^{2}$ close to the original presents technical issues. We refer readers to the proof of Lemma A.2 for an idea of the method of perturbation in this proof. The third step uses the compactness of $M$ to note that there are finitely many hyperbolic singularities for vector field $X$, and hence there is a minimal distance between them. By using nonoverlapping bump functions one can show that the perturbation at each singularity can be done simultaneously. This shows the density part of B2.

Openness follows from the openness of $\mathcal{A}$ in the set of all linear operators. Let $X$ be a vector field with $X(x)=0$ such that $D X(x) \in \mathcal{A}$, and let $\delta>0$ be such that $B \in \mathcal{A}$ when $B$ is within $\delta$ of $D X(x)$. Then, for any smooth $Y$ with $Y(x)=0$ sufficiently close to $X$, the difference in derivatives $D X(x)-D Y(x)$ is smaller than $\delta$.

Proof of Theorem 3.3. We will show that if conditions B1 and B2 are fulfilled for $\psi^{t}$ and $T$, then the diffeomorphism $\phi^{T}: M \rightarrow M$ will fulfill properties A1 and A2, making $\Phi_{\left(\phi^{T}, \varphi\right)}: M \rightarrow \mathbb{R}^{2 m+1}$ generically an embedding by Takens' Theorem 3.1. Since condition B1 implies no periodic points of $\psi^{t}$ with periods $k T$ for $1 \leq k \leq n, n \in \mathbb{N}$ fixed, then the only closed orbits in the set $\left\{\phi^{T}(x),\left(\phi^{T}\right)^{2}(x), \ldots,\left(\phi^{T}\right)^{n}(x)\right\}$ are fixed points of period 1 for any $x \in M$. Since generically there are only a finite number of singularities of $\psi^{t}$, there can be only a finite number of fixed points of $\phi^{T}$. So B1 implies A1. Since $\phi^{T}$ has only fixed points (i.e., periodic points of period 1), then A2 requires only that the derivative of $\phi^{T}$ at the fixed points have distinct eigenvalues not on the unit circle. This is exactly what was proved in condition B2.

Now we extend these results to the smaller set of flows with a fixed component graph CG. Given CG and a compact manifold $M$, we build the corresponding upper set lattice, $U L$, and manifold filtration $\mathbb{M}$. For any two upper sets satisfying $U<V$, denote the dimensions of the compact manifolds $M_{U}, M_{V} \in \mathbb{M}$ by $n_{U}, n_{V}$, respectively, and their associated flows by $\psi_{U}^{t}, \psi_{V}^{t}$. Recall that since $U<V$, there is an induced projection $\pi_{V U}$ such that $\pi_{V U}\left(M_{V}\right)=M_{U}$ and $\pi_{V U} \circ \psi_{V}^{t}=\psi_{U}^{t} \circ \pi_{V U}$. Recall also Definition 3.5, where we define the subset $\mathcal{P}_{\mathrm{CG}} \subset C^{2}(M, M)$ to consist of those flows where $\pi_{V U} \circ \psi_{V}^{t}=\psi_{U}^{t} \circ \pi_{V U}$ holds for any pair of upper sets in $U L$ satisfying $U<V$ for a fixed pair (CG, $M$ ). Our goal is to prove that conditions B1 and B2 are generic in this smaller set $\mathcal{P}_{\mathrm{CG}} \times \mathbb{R}^{+}$.

To prove this we first consider the simplest possible upper set lattice $U L$ which consists of two sets $U, V$ with $U<V$. Arguing openness is straightforward, but arguing density requires 
substantial work. We will use this result and induction on $U L$ to show genericity of B1 and B2 in $\mathcal{P}_{\text {CG. }}$.

Theorem A.2. Consider a component graph CG with only two strongly connected components, so that the upper set lattice is composed of sets $U$ and $V$ with $\emptyset<U<V$. Then the set of $\left(\psi_{V}^{t}, T\right) \in \mathcal{P}_{\mathrm{CG}} \times \mathbb{R}^{+}$where $\psi_{V}^{t}$ has no periodic orbits of period $j T$ with $j \in\left\{1,2, \ldots, 2 n_{V}+1\right\}$ (property $\mathrm{B} 1$ ) is open and dense in $\mathcal{P}_{\mathrm{CG}} \times \mathbb{R}^{+}$.

Before we prove Theorem A.2 we need a lemma which will allow us to transfer the relationship between $U$ and $V$ locally to coordinate charts. Let $\left(c_{i}, Q_{i}\right)$ be an atlas mapping $M_{V}$ locally to $\mathbb{R}^{n_{V}}$ and $\left(d_{i}, P_{i}\right)$ an atlas from $M_{U}$ to $\mathbb{R}^{n_{U}}$. Given an arbitrary point $v \in M_{V}$, consider an open neighborhood $O \subset M_{V}$ small enough so that the following hold:

1. $O \subset Q_{j}$ and $\psi_{V}^{t}(O) \subset Q_{j}$ for a fixed index $j$ and a small time $t$.

2. $\pi_{V U}(O) \subset P_{j}$ and $\psi_{U}^{t}\left(\pi_{V U}(O)\right) \subset P_{j}$ for a fixed index which we may take to be $j$ without loss of generality, and a small time $t$.

There are three locally induced maps to consider:

$$
\begin{aligned}
\tilde{\psi}_{V}^{t} & \equiv c_{j} \circ \psi_{V}^{t} \circ c_{j}^{-1} \in C^{2}\left(\mathbb{R}^{n_{V}}\right), \\
\tilde{\psi_{U}^{t}} & \equiv d_{j} \circ \psi_{U}^{t} \circ d_{j}^{-1} \in C^{2}\left(\mathbb{R}^{n_{U}}\right), \\
\tilde{\pi_{j}} & \equiv d_{j} \circ \pi_{V U} \circ c_{j}^{-1} \in C^{\infty}\left(\mathbb{R}^{n_{V}}, \mathbb{R}^{n_{U}}\right) .
\end{aligned}
$$

The induced projection-like function is $C^{\infty}$ because we assume that the manifolds $M_{U}$ and $M_{V}$ are $C^{\infty}$, which then implies that $\pi_{V U}$ is $C^{\infty}$.

Lemma A.3. Let $\left(c_{i}, Q_{i}\right)$ be an atlas mapping $M_{V}$ locally to $\mathbb{R}^{n_{V}}$ and $\left(d_{i}, P_{i}\right)$ an atlas from $M_{U}$ to $\mathbb{R}^{n_{U}}$, as above. Then

$$
\tilde{\pi_{j}} \circ \tilde{\psi_{V}^{t}}=\tilde{\psi_{U}^{t}} \circ \tilde{\pi_{j}}
$$

Proof. We note that

$$
\begin{aligned}
\tilde{\pi}_{j} \circ c_{j} & =d_{j} \circ \pi_{V U}, \\
\pi_{V U} \circ c_{j}^{-1} & =d_{j}^{-1} \circ \tilde{\pi}_{j} .
\end{aligned}
$$

Then

$$
\begin{aligned}
\tilde{\pi_{j}} \circ \tilde{\psi_{V}^{t}} & =\tilde{\pi}_{j} \circ c_{j} \circ \psi_{V}^{t} \circ c_{j}^{-1}, \\
& =d_{j} \circ \pi_{V U} \circ \psi_{V}^{t} \circ c_{j}^{-1}, \\
& =d_{j} \circ \psi_{U}^{t} \circ \pi_{V U} \circ c_{j}^{-1}, \\
& =d_{j} \circ \psi_{U}^{t} \circ d_{j}^{-1} \circ \tilde{\pi}_{j}, \\
& =\tilde{\psi_{U}^{t}} \circ \tilde{\pi}_{j} .
\end{aligned}
$$

Therefore the projection relationship holds locally near any $c_{i}(v)$ for $v \in M_{V}$.

Proof of Theorem A.2. Given $T$, we denote periodic orbits of period $j T$ with $j \in$ $\left\{1,2, \ldots, 2 n_{V}+1\right\}$ as bad periodic orbits.

Open. The set of $\left(\psi_{V}^{t}, T\right) \in C^{2}\left(M_{V}, M_{V}\right) \times \mathbb{R}^{+}$fulfilling condition B1 (i.e., there are no bad periodic orbits) is open; therefore it is relatively open in $\mathcal{P}_{\mathrm{CG}} \times \mathbb{R}^{+}$.

Copyright (C) by SIAM. Unauthorized reproduction of this article is prohibited. 
Dense. The set of $\left(\psi_{U}^{t}, T\right) \in C^{2}\left(M_{U}, M_{U}\right) \times \mathbb{R}^{+}$fulfilling condition B1 is open and dense; i.e., there are no bad periodic orbits for pairs $\left(\psi_{U}^{t}, T\right)$ in the generic set. Given a generic choice of $\left(\psi_{U}^{t}, T\right)$, the key is to show that the set of $\psi_{V}^{t}$ with no bad periodic orbits in $T$ is dense in the set of $\psi_{V}^{t}$ satisfying $\pi_{V U} \circ \psi_{V}^{t}=\psi_{U}^{t} \circ \pi_{V U}$. To do this, we assume that there exists a bad periodic orbit in $\psi_{V}^{t}$ and construct an arbitrarily close perturbation in $\mathcal{P}_{\text {CG }}$ with no bad periodic orbits that respects the projection relationship.

We observe that if $\psi_{V}^{t}$ has a bad periodic orbit, it must occur at an equilibrium point of $\psi_{U}^{t}$, given a generic choice of $\psi_{U}^{t}$ and $T$. Since there are a finite number of equilibrium points of $\psi_{U}^{t}$, we need to make at most a finite number of perturbations to $\psi_{V}^{t}$ to ensure that there are no bad periodic orbits of $\psi_{V}^{t}$ with the same $T$.

Consider a single equilibrium point of $\psi_{U}^{t}$ giving rise to a bad periodic orbit of $\psi_{V}^{t}$. We denote that equilibrium point by $u_{e}$. We will show that for any $\epsilon>0$, there exists a flow $\xi_{V}^{t} \in \mathcal{P}_{\mathrm{CG}}$ and an open neighborhood of $u_{e}, P \subset M_{U}$, such that

1. $\xi_{V}^{t}$ has no bad periodic orbits in the set $S=\left\{v: v \in M_{V}, \pi_{V U}(v) \in P\right\}$;

2. $\xi_{V}^{t}$ is a local perturbation of $\psi_{V}^{t}$; i.e., $\xi_{V}^{t}=\psi_{V}^{t}$ for all $v \in M_{V} \backslash S$;

3. $\xi_{V}^{t}$ can be arbitrarily close to $\psi_{V}^{t}$; i.e., $\left\|\xi_{V}^{t}-\psi_{V}^{t}\right\|<\epsilon$ on $S$ in the $C^{2}$ norm.

Using the result and notation of Lemma A.3, we may prove 1-3 for the flows $\tilde{\psi}_{V}^{t} \in$ $C^{2}\left(\mathbb{R}^{n_{V}}, \mathbb{R}^{n_{V}}\right)$ and $\tilde{\psi_{U}^{t}} \in C^{2}\left(\mathbb{R}^{n_{U}}, \mathbb{R}^{n_{U}}\right)$, and the result can be passed through the charts back to the original flows.

If the $C^{\infty}$ projection $\pi_{V U}: M_{V} \rightarrow M_{U}$ is restricted to the domain $M_{V}$, then the set $N:=\pi_{V U}^{-1}\left(u_{e}\right)$ is generically a compact submanifold of $M_{V}$, because $u_{e}$ is generically a regular value of $\pi_{V U}$ by the Morse-Sard theorem [8]. Clearly $N$ has an atlas $\left(c_{i}, O_{i}\right)$ with $O_{i}=Q_{i} \cap N$, consistent with the atlas $\left(c_{i}, Q_{i}\right)$ of $M_{V}$, and is locally diffeomorphic to $\mathbb{R}^{n_{V}-n_{U}}$ (see [8, pp. 1314]). The flow $\psi_{V}^{t}$ induces a flow $\left.\psi_{V}^{t}\right|_{u_{e}}$ on the manifold $N$, which exhibits the bad periodic orbit.

By the genericity of $\mathrm{B} 1$ in the set $C^{2}$ flows on the compact manifold $N$, we may choose a $C^{2}$ flow $\kappa^{t}: N \rightarrow N$ with no bad periodic orbits such that $\left\|\kappa^{t}-\left.\psi_{V}^{t}\right|_{u_{e}}\right\|<\mu$ in the $C^{2}$ norm, where $\mu$ is sufficiently small (exact condition to be determined later). Let $\tilde{\kappa}^{t}=c_{j} \circ \kappa^{t} \circ c_{j}^{-1}$ be the locally induced flow. The condition $\left\|\kappa^{t}-\left.\psi_{V}^{t}\right|_{u_{e}}\right\|<\mu$ in the $C^{2}$ norm means that ||$\tilde{\kappa}^{t}-\left.\tilde{\psi}_{V}^{t}\right|_{u_{e}} \|<\mu$ in the $C^{2}$ norm on $\mathbb{R}^{n_{V}-n_{U}}$ for every open set $O_{i}$ in the atlas of $N$. We shall assume for the remainder of the proof that the mapped flows $(\tilde{\psi}, \tilde{\kappa})$ are $C^{\infty}$ functions on their respective domains, since $C^{2}$ functions may be approximated arbitrarily closely with $C^{\infty}$ functions.

There exists a small neighborhood $N_{U} \subset P_{i} \subset M_{U}$ containing $u_{e}$ that contains no other limit sets of $\psi_{U}^{t}$, where $P_{i}$ is an open set in the atlas $\left(d_{i}, P_{i}\right)$ of $M_{U}$. Without loss of generality, assume that $d_{j}\left(u_{e}\right)=0 \in \mathbb{R}^{n_{U}}$; that is, the equilibrium point of interest is mapped to the origin. We shall choose $\delta \ll 1$ sufficiently small that the $\delta$-ball in $\mathbb{R}^{n_{U}}$ satisfies $d_{j}^{-1}\left(\overline{B_{\delta}(0)}\right) \subset$ $N_{U}$. Further conditions on the smallness of $\delta$ will be imposed later in the proof.

For each $v \in N$, there exists a small neighborhood $N_{v} \subset O_{i}$ for some $O_{i}$ in the atlas of $N$. There is a compact set $C_{v}$ such that $v \in C_{v} \subset N_{v}$. Let $\overline{B_{v}}=c_{i}\left(C_{v}\right) \in \mathbb{R}^{n_{V}-n_{U}}$.

By Lemma A.3, we may write $\tilde{\psi}_{U}^{t} \equiv g^{t}(x)$ and $\tilde{\psi}_{V}^{t} \equiv\left(g^{t}(x), f^{t}(x, y)\right)$ for $x \in \overline{B_{\delta}(0)} \subset$ $\mathbb{R}^{n_{U}}, y \in \overline{B_{v}} \subset \mathbb{R}^{n_{V}-n_{U}}$. Then $f^{t}(0, y)$ and $\tilde{\kappa}^{t}(0, y)$ have the domain $\{0\} \times \overline{B_{v}}$, where the input $y$ for both functions is generated from the same atlas. We emphasize that $f^{t}$ and $\tilde{\kappa}^{t}$ are

Copyright (c) by SIAM. Unauthorized reproduction of this article is prohibited. 
$C^{\infty}$ on compact domains, which will be important when we take derivatives of Taylor series later on.

We extend $\tilde{\kappa}^{t}$ to a neighborhood of $0 \in \mathbb{R}^{n_{V}}$ by adding the Taylor expansion of $f^{t}$ and concatenating with $g^{t}$ on $\mathbb{R}^{n_{U}}$ :

$$
\begin{aligned}
k^{t}(x, y) & =\left(g^{t}(x), \tilde{\kappa}^{t}(0, y)\right)+\left(0, x^{T} D_{x} f^{t}(0, y)+\frac{1}{2} x^{T} D_{x x} f^{t}(0, y) x\right) \\
& =\left(g^{t}(x), \tilde{\kappa}^{t}(y)+\sum_{i=1}^{n_{U}} x_{i} \frac{\partial f^{t}}{\partial x_{i}}(0, y)+\sum_{i=1}^{n_{U}} \sum_{j=1}^{n_{U}} \frac{x_{i} x_{j}}{2} \frac{\partial^{2} f^{t}}{\partial x_{i} \partial x_{j}}(0, y)\right),
\end{aligned}
$$

where $D_{x}$ is the $n_{U} \times n_{V}$ Jacobian of $f^{t}$ with respect to the vector $x$. Likewise $D_{x x}$ is the $n_{U} \times n_{V} \times n_{U}$ Hessian of $f^{t}$.

There exists a bump function $h \in C^{\infty}\left(\mathbb{R}^{n_{U}},[0,1]\right)$ such that $h(x)=1$ on the closed ball $\overline{B_{\delta / 2}(0)}$ and $h(x)=0$ on the closed set $\mathbb{R}^{n_{U}} \backslash B_{\delta}(0)$. We are now ready to construct a function that (locally in $\mathbb{R}^{n_{V}}$ ) fulfills properties $1-3$. Let

$$
\tilde{\xi}_{V}^{t}(x, y)=(1-h(x)) \tilde{\psi}_{V}^{t}(x, y)+h(x) k^{t}(x, y) .
$$

Properties 1 and 2 are satisfied by construction. Since $\tilde{\xi}_{V}^{t}(0, y)=\left(0, \tilde{\kappa}^{t}(0, y)\right)$ and there are no other equilibrium points of $\tilde{\psi}_{U}^{t}$ in the $\delta$-neighborhood of $0, \tilde{\xi}_{V}^{t}(x, y)$ has no bad periodic orbits for $x \in B_{\delta}(0)$. And clearly $\tilde{\xi}_{V}^{t}(x, y)=\tilde{\psi}_{V}^{t}(x, y)$ for any $x$ outside of the $\delta$-ball. Notice also that $\tilde{\xi}_{V}^{t}$ is $C^{2}$ and respects the projection relationship $\tilde{\pi} \circ \tilde{\xi}_{V}^{t}=\tilde{\psi}_{U}^{t} \circ \tilde{\pi}$.

The work is in proving property 3 . Consider

$$
\begin{aligned}
\tilde{\xi}_{V}^{t}(x, y)-\tilde{\psi}_{V}^{t}(x, y)= & h(x)\left(0, \tilde{\kappa}^{t}(0, y)+x^{T} D_{x} f^{t}(0, y)+\frac{1}{2} x^{T} D_{x x} f^{t}(0, y) x-f^{t}(x, y)\right) \\
= & h(x)\left(0, \tilde{\kappa}^{t}(0, y)-f^{t}(0, y)\right) \\
& +h(x)\left(0, f^{t}(0, y)+x^{T} D_{x} f^{t}(0, y)+\frac{1}{2} x^{T} D_{x x} f^{t}(0, y) x-f^{t}(x, y)\right) \\
:= & h(x)\left(0, \tilde{\kappa}^{t}(0, y)-f^{t}(0, y)\right)+h(x)(0, T(x, y)) .
\end{aligned}
$$

The idea is that the first term is small by the choice of $\tilde{\kappa}^{t}$, and the second term is small because it is the difference of a function and its Taylor series. However, there are technical details that come from using the $C^{2}$ norm, $\|f\|=\sum_{|\alpha| \leq 2} \sup _{x \in B}\left|f^{\alpha}(x)\right|$, where each $\alpha$ is a multi-index. We will abuse notation slightly and allow $\|\cdot\|$ to represent the $C^{2}$ norm over $\mathbb{R}^{p}$ or $M$ for various Euclidean spaces or manifolds as indicated by context.

The first term in (10) is easier to handle, but it imposes a constraint on $\mu$ in terms of $\delta$, and $\delta$ has independent constraints arising from the second term. Therefore, we address the second term before the first.

First we note that

$$
\sum_{i=1}^{n_{U}} \sup _{x \in \overline{B_{\delta}(0)}}\left|\frac{\partial h(x)}{\partial x_{i}}\right|=\frac{M_{1}}{\delta}
$$

Copyright $\odot$ by SIAM. Unauthorized reproduction of this article is prohibited. 
for some constant $M_{1}$. This is because any bump function $b(x)$ with support on the unit ball $\overline{B_{1}(0)}$ will have the sum of the magnitudes of the first derivatives bounded by some number $M_{1}$, and $h(x)$ can be constructed by $h(x):=b(x / \delta)$. Likewise, the second derivative of $h(x)$ is bounded by $M_{2} / \delta^{2}$.

For the second term in (10), we consider each part of the $C^{2}$ norm separately.

$$
\begin{aligned}
& \sup _{x \in \overline{B_{\delta}(0)}, y \in \overline{B_{v}}}|h(x) T(x, y)| \\
\leq & \sup _{x \in \overline{B_{\delta}(0)}}|h(x)| \sup _{x \in \overline{B_{\delta}(0)}, y \in \overline{B_{v}}}\left|f^{t}(0, y)+x^{T} D_{x} f^{t}(0, y)+\frac{1}{2} x^{T} D_{x x} f^{t}(0, y) x-f^{t}(x, y)\right| \\
\leq & \sup _{x \in \overline{B_{\delta}(0)}, y \in \overline{B_{v}}}\left|\sum_{|\alpha|=3} \frac{x^{\alpha}}{|\alpha| !} \frac{\partial^{|\alpha|} f^{t}}{\partial x^{\alpha}}(x, y)\right| \delta^{3} \\
\leq & M_{3} \delta^{3}
\end{aligned}
$$

by Taylor's theorem over $x$ in the $\delta$-ball. $M_{3}$ is the result of taking the supremum of the Taylor coefficients over the compact set $\overline{B_{v}}$. From now on we will skip the explicit step of taking the supremum of the Taylor coefficients, but it occurs implicitly at every invocation of Taylor's theorem below. Additionally, when we take the sup below, it will occur over the set $\left\{x \in \overline{B_{\delta}(0)}, y \in \overline{B_{v}}\right\}$, but we will omit the set in our notation to improve readability.

$$
\begin{aligned}
\sum_{i=1}^{n_{U}} \sup \mid \frac{\partial h}{\partial x_{i}} T(x, y) & +h(x) \frac{\partial T}{\partial x_{i}}\left|+\sum_{k=1}^{n_{V}-n_{U}} \sup \right| h(x) \frac{\partial T}{\partial y_{k}} \mid \\
& \leq \sum_{i=1}^{n_{U}} \sup \left|\frac{\partial h}{\partial x_{i}}\right||T(x, y)|+\sum_{i=1}^{n_{U}} \sup \left|\frac{\partial T}{\partial x_{i}}\right|+\sum_{k=1}^{n_{V}-n_{U}} \sup \left|\frac{\partial T}{\partial y_{k}}\right| \\
& \leq \frac{M_{1}}{\delta} M_{3} \delta^{3}+\sum_{i=1}^{n_{U}} \sup \left|\frac{\partial f^{t}}{\partial x_{i}}(0, y)+\sum_{j=1}^{n_{U}} x_{j} \frac{\partial^{2} f^{t}}{\partial x_{i} \partial x_{j}}(0, y)-\frac{\partial f^{t}}{\partial x_{i}}(x, y)\right| \\
& +\sum_{k=1}^{n_{V}-n_{U}} \sup \mid \frac{\partial f^{t}}{\partial y_{k}}(0, y)+\sum_{i=1}^{n_{U}} x_{i} \frac{\partial^{2} f^{t}}{\partial y_{k} \partial x_{i}}(0, y) \\
& +\sum_{i=1}^{n_{U}} \sum_{j=1}^{n_{U}} \frac{x_{i} x_{j}}{2} \frac{\partial^{3} f^{t}}{\partial y_{k} \partial x_{i} \partial x_{j}}(0, y)-\frac{\partial f^{t}}{\partial y_{k}}(x, y) \mid \\
& \leq M_{1} M_{3} \delta^{2}+M_{4} \delta^{2}+M_{5} \delta^{3},
\end{aligned}
$$

where the last two estimates come from Taylor's theorem on $\frac{\partial f^{t}}{\partial x_{i}}$ and $\frac{\partial f^{t}}{\partial y_{k}}$. 
The second derivative estimate is as follows:

$$
\begin{aligned}
\sum_{j=1}^{n_{U}} \sum_{i=1}^{n_{U}} \sup \mid & \frac{\partial^{2} h}{\partial x_{j} \partial x_{i}} T(x, y)+2 \frac{\partial h}{\partial x_{i}} \frac{\partial T}{\partial x_{j}}+h(x) \frac{\partial^{2} T}{\partial x_{j} \partial x_{i}}\left|+\sum_{j=1}^{n_{U}} \sum_{k=1}^{n_{V}-n_{U}} \sup \right| \frac{\partial h}{\partial x_{j}} \frac{\partial T}{\partial y_{k}}+h(x) \frac{\partial^{2} T}{\partial x_{j} \partial y_{k}} \mid \\
& +\sum_{\ell=1}^{n_{V}-n_{U}} \sum_{i=1}^{n_{U}} \sup \left|\frac{\partial h}{\partial x_{i}} \frac{\partial T}{\partial y_{\ell}}+h(x) \frac{\partial^{2} T}{\partial y_{\ell} \partial x_{i}}\right|+\sum_{\ell=1}^{n_{V}-n_{U}} \sum_{k=1}^{n_{V}-n_{U}} \sup \left|h(x) \frac{\partial^{2} T}{\partial y_{\ell} \partial y_{k}}\right| \\
\leq & M_{2} M_{3} \delta+2 M_{1} M_{4} \delta+2 M_{1} M_{5} \delta^{2}+\sum_{j=1}^{n_{U}} \sum_{i=1}^{n_{U}} \sup \left|\frac{\partial^{2} T}{\partial x_{j} \partial x_{i}}\right| \\
& +2 \sum_{j=1}^{n_{U}} \sum_{k=1}^{n_{V}-n_{U}} \sup \left|\frac{\partial^{2} T}{\partial x_{j} \partial y_{k}}\right|+\sum_{\ell=1}^{n_{V}-n_{U}} \sum_{k=1}^{n_{V}-n_{U}} \sup \left|\frac{\partial^{2} T}{\partial y_{\ell} \partial y_{k}}\right|
\end{aligned}
$$

where

$$
\begin{aligned}
& \sum_{j=1}^{n_{U}} \sum_{i=1}^{n_{U}} \sup \left|\frac{\partial^{2} T}{\partial x_{j} \partial x_{i}}\right|=\sum_{j=1}^{n_{U}} \sum_{i=1}^{n_{U}} \sup \left|\frac{\partial^{2} f^{t}}{\partial x_{i} \partial x_{j}}(0, y)-\frac{\partial^{2} f^{t}}{\partial x_{j} \partial x_{i}}(x, y)\right| \leq M_{6} \delta \\
& \sum_{j=1}^{n_{U}} \sum_{k=1}^{n_{V}-n_{U}} \sup \left|\frac{\partial^{2} T}{\partial x_{j} \partial y_{k}}\right| \\
& \quad=\sum_{j=1}^{n_{U}} \sum_{k=1}^{n_{V}-n_{U}} \sup \left|\frac{\partial^{2} f^{t}}{\partial x_{j} \partial y_{k}}(0, y)+\sum_{i=1}^{n_{U}} x_{i} \frac{\partial^{3} f^{t}}{\partial y_{k} \partial x_{i} \partial x_{j}}(0, y)-\frac{\partial^{2} f^{t}}{\partial x_{j} \partial y_{k}}(x, y)\right| \\
& \leq M_{7} \delta^{2}
\end{aligned}
$$

$$
\begin{aligned}
\sum_{\ell=1}^{n_{V}-n_{U}} \sum_{k=1}^{n_{V}-n_{U}} \sup \left|\frac{\partial^{2} T}{\partial y_{\ell} \partial y_{k}}\right| & \\
= & \sum_{\ell=1}^{n_{V}-n_{U}} \sum_{k=1}^{n_{V}-n_{U}} \sup \mid \frac{\partial^{2} f^{t}}{\partial y_{\ell} \partial y_{k}}(0, y)+\sum_{i=1}^{n_{U}} x_{i} \frac{\partial^{3} f^{t}}{\partial y_{\ell} \partial y_{k} \partial x_{i}}(0, y) \\
& \quad+\sum_{i=1}^{n_{U}} \sum_{j=1}^{n_{U}} \frac{x_{i} x_{j}}{2} \frac{\partial^{4} f^{t}}{\partial y_{\ell} \partial y_{k} \partial x_{i} \partial x_{j}}(0, y)-\frac{\partial^{2} f^{t}}{\partial y_{\ell} \partial y_{k}}(x, y) \mid \\
\leq & M_{8} \delta^{3} .
\end{aligned}
$$

Putting everything together,

$$
\begin{aligned}
\|h(x) T(x, y)\| \leq & M_{3} \delta^{3}+M_{1} M_{3} \delta^{2}+M_{4} \delta^{2}+M_{5} \delta^{3}+M_{2} M_{3} \delta \\
& +2 M_{1} M_{4} \delta+2 M_{1} M_{5} \delta^{2}+M_{6} \delta+2 M_{7} \delta^{2}+M_{8} \delta^{3} \\
=\delta & \left(M_{2} M_{3}+2 M_{1} M_{4}+M_{6}\right)+\delta^{2}\left(M_{1} M_{3}+M_{4}+2 M_{1} M_{5}+2 M_{7}\right) \\
& +\delta^{3}\left(M_{3}+M_{5}+M_{8}\right) .
\end{aligned}
$$

Copyright (C) by SIAM. Unauthorized reproduction of this article is prohibited. 
The parameter $\delta$ must be chosen small enough so that the term above is less than $\epsilon / 2$.

The first term reduces to

$$
\begin{aligned}
\left\|h(x)\left(\tilde{\kappa}^{t}(0, y)-f^{t}(0, y)\right)\right\| & \leq\|h(x)\|\left\|\tilde{\kappa}^{t}(0, y)-f^{t}(0, y)\right\| \\
& \leq\left(1+\frac{M_{1}}{\delta}+\frac{M_{2}}{\delta^{2}}\right) \mu \\
& <\epsilon / 2,
\end{aligned}
$$

where we enforce the last inequality by taking $\mu \ll \epsilon$ and $\mu \ll \delta$. This completes the proof of property 3 .

The local perturbation $\tilde{\xi}^{t}(x, y) \in C^{\infty}\left(\mathbb{R}^{n_{V}}, \mathbb{R}^{n_{V}}\right)$ may be mapped back to the manifold via $\xi_{V}^{t}=c_{j}^{-1} \circ \tilde{\xi}^{t} \circ c_{j}$, again using the language of Lemma A.3. This process was done for an arbitrary $v \in N$, so it holds for all points $p \in S=\left\{p: p \in M_{V}, \pi_{V U}(p) \in P\right\}$, where $P=d_{j}^{-1}\left(B_{\delta}(0)\right)$. Then properties 1 and 3 hold in $S$, and no changes were introduced outside of this set, so that property 2 holds in $M_{V} \backslash S$.

Now suppose $\psi_{V}^{t}$ has more than one bad periodic orbit (i.e., $\psi_{U}^{t}$ has more than one bad equilibrium point), noting that there may be only a finite number of bad periodic orbits. Since the flows $\xi_{V}^{t}$ are local perturbations on disjoint sets, a finite number of them may summed together into a single flow satisfying conditions 1-3.

Theorem A.4. Consider a component graph CG with only two strongly connected components, so that the upper set lattice is composed of sets $U$ and $V$ with $\emptyset<U<V$. Then a subset of elements $\left(\psi_{V}^{t}, T\right)$ that satisfies property $\mathrm{B} 2$ (at an equilibrium point, the eigenvalues of the Jacobian are distinct with moduli different from 1) is open and dense in $\mathcal{P}_{\mathrm{CG}} \times \mathbb{R}^{+}$.

Proof.

Open. As before, the set of $\left(\psi_{V}^{t}, T\right)$ fulfilling condition B2 is open in the set of $C^{2}\left(M_{V}, M_{V}\right)$ $\times \mathbb{R}^{+}$. Therefore it is relatively open in the set $\mathcal{P}_{\mathrm{CG}} \times \mathbb{R}^{+}$.

Dense. We outline the proof of density, which combines Theorem A.1 and the ideas of the proof of Theorem A.2. By Theorem A.1 there is a generic choice of $\psi_{U}^{t} \in C^{2}\left(M_{U}, M_{U}\right)$ which satisfies B2. In particular, $\psi_{U}^{t}$ has finitely many hyperbolic equilibria which have distinct eigenvalues. Take now an equilibrium $z$ of a flow $\psi_{V}^{t} \in \mathcal{P}_{\mathrm{CG}}$. Passing to local coordinates as in the previous proof, the flow in local coordinates $\tilde{\psi}_{V}^{t}$ can be written as

$$
\tilde{\psi}_{V}^{t}=\left(g^{t}(x), f^{t}(x, y)\right)
$$

It follows that the equilibrium $\tilde{z}=(\tilde{x}, \tilde{y})$ is also an equilibrium of $\tilde{\psi}_{U}^{t}$. The key observation is that the linearization $A$ at $\tilde{z}$ has a lower triangular structure

$$
A=\left[\begin{array}{ll}
B & 0 \\
D & C
\end{array}\right]
$$

where $B=D g^{t}(\tilde{x})$ and $C=\frac{D f^{t}}{D y}(\tilde{x}, \tilde{y})$. We note that the eigenvalues of $A$ are the union of eigenvalues of $B$ and eigenvalues of $C$. As in the proof of Theorem A.2, we can perturb $C$ in the submanifold $N$, while keeping $B$ unchanged. Since $N$ is generically a manifold of dimension $n_{V}-n_{U}$, a small perturbation of $C$ will guarantee hyperbolicity and distinct eigenvalues for $C$ as well as the entire matrix $A$. Further, using the same methods as in Theorem A.2, we can 
localize this perturbation to function $\tilde{f}^{t}$ to a neighborhood of $\tilde{x}$. Since there are only finitely many equilibria $\tilde{z}$, these local perturbations can be composed to arrive at perturbation of the flow $\psi_{V}^{t}$ that satisfies B2.

We are almost ready to prove the main result. In the proof we will require the following definition.

Definition A.5. For a given upper set lattice $U L$, the least elements of $U L$ are the nonempty upper sets $U_{1}, \ldots, U_{n}$ such that for every $V \in U L$, the meet (greatest lower bound) of $V$ and $U_{i}, V \vee U_{i}$, is either $U_{i}$ or $\emptyset$. That is, each $U_{i}$ consists of a single strongly connected component in the component graph CG with only outgoing edges.

Theorem A.6. Consider a flow $\psi^{t}$ on a smooth, compact manifold $M$ with the corresponding component graph CG. Then conditions B1 and B2 hold on an open and dense set of $\left(\psi^{t}, T\right) \in$ $\mathcal{P}_{\mathrm{CG}} \times \mathbb{R}^{+}$.

Proof. The upper set lattice $U L$ associated with CG may be divided into levels $L_{i}$, where the empty set is the only element in $L_{0}$, the least elements of $U L$ form the level $L_{1}$, and the top level, composed of a single element, is $L_{N}$. We present an induction proof on the levels of $U L$.

Let $m$ be the dimension of the manifold $M$. For each least element $U_{i}$ in the level $L_{1}$, $\left(\psi_{U_{i}}^{t}, T_{i}\right)$ may be chosen from $C^{2}\left(M_{U}, M_{U}\right) \times \mathbb{R}^{+}$to generically satisfy B1 and B2. Specifically for $\mathrm{B} 1$, periodic orbits of period $k T_{i}$ do not exist for $k \leq 2 m+1$, a number depending on the dimension of $M$, not on $M_{U}$. Since the number of sets in $L_{1}$ is finite, generically each $T_{i}$ satisfies all the $\psi_{U}^{t}$, and we choose one $T$ for the whole level.

Now consider $L_{2}$. Every element in the upper set lattice is the immediate successor of either one or two elements as in the following diagram:
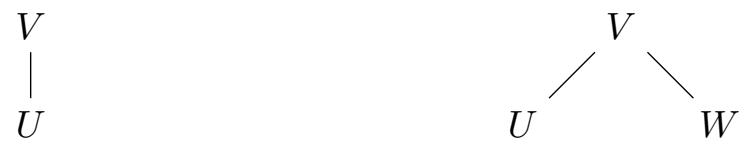

We proved in Lemmas A.2 and A.4 that for the case on the left, a generic choice of $\left(\psi_{U}^{t}, T\right)$ implies that $\psi_{V}^{t}$ generically satisfies B1 and B2 for the same choice of $T$. For the case on the right, if $|U| \times|W|$ is taken as a product space, then $M_{U} \times M_{V}$ is a manifold and the results of Lemmas A.2 and A.4 immediately apply as well (indeed this is true for any $V$ with a finite number of incoming edges, so we are not limited to the special case of an upper set lattice). Therefore the flows associated with upper sets in $L_{2}$ generically satisfy B1 and B2.

It is easily seen by the same argument that if B1 and B2 hold generically for the flows associated with the upper sets in $L_{i-1}$, then they hold generically for flows in $L_{i}$, where the projection relationships between the levels are satisfied. Since all flows satisfy B1 for $k \leq 2 m+1$, the requirements for Takens' theorem hold at all levels of $U L$. And since $T$ is the same for all flows, then B1 and B2 hold generically in $\mathcal{P}_{\mathrm{CG}} \times \mathbb{R}^{+}$.

An immediate consequence of Theorem A.6 is Theorem 3.6 in section 3, which we now prove for flows only.

Proof of Theorem 3.6 for flows. Let $\mathfrak{G} \subset \mathcal{P}_{\mathrm{CG}} \times \mathbb{R}^{+}$be the open and dense set established in Theorem A.6, so that $\left(\psi^{t}, T\right) \in \mathfrak{G}$ satisfies properties B1 and B2, and $\psi^{t}$ fulfills the projection relationships $\pi_{V U} \circ \psi_{V}^{t}=\psi_{U}^{t} \circ \pi_{V U}$ for $U<V$ in the upper set lattice. Fix a point on the manifold $p \in M$. Then by Theorem 3.4 there exists a residual set $G(p) \subset C^{2}(M, M) \times \mathbb{R}^{+}$ 
such that $\left(\psi^{t}, T\right) \in G(p)$ satisfies B1 and B2, and the limit points of the flow starting at $p$ are bijectively mapped to the limit points of the embedding constructed from a generic observation function. Then the set

$$
\mathrm{G}_{\mathcal{P}}:=\mathfrak{G} \cap G(p)
$$

is a residual subset of $\mathcal{P}_{C G} \times \mathbb{R}^{+}$simultaneously satisfying the requirements of Theorem 3.4 and respecting the structure of the component graph CG. Since the projection of the positive limit points of $\psi^{t}$ onto $M_{U}$ are the positive limit points of $\psi_{U}^{t}$, the pair $\left(\psi_{U}^{t}, T\right)$ generically satisfies the requirements of Theorem 3.4 while ensuring that $\psi_{U}^{t}=\pi_{U} \circ \psi^{t}$.

Theorems A.6 and 3.6 allow us to apply Theorems 3.3 and 3.4 to each element $\left(\psi_{V}^{t}, M_{V}\right)$ of the filtration of $\left(\psi^{t}, M\right)$, as we did in section 3. We assume that the proof for genericity of conditions $\mathrm{A} 1$ and $\mathrm{A} 2$ for diffeomorphisms $\phi \in \mathcal{P}_{\mathrm{CG}} \subset D^{2}(M, M)$ may be similarly proven, which would allow the application of Theorem 3.1 to the filtration as well.

Acknowledgments. We would like to thank Dr. Tim Sauer and an anonymous reviewer for helpful comments that improved the quality of this paper. We also thank Dr. Jarek Kwapisz for substantial assistance with the proof of Theorem A.2.

\section{REFERENCES}

[1] V. I. Arnol'D, Geometrical Methods in the Theory of Ordinary Differential Equations, Springer-Verlag, New York, 1983.

[2] T. Buzug And G. Pfister, Optimal delay time and embedding dimension for delay-time coordinates by analysis of the global static and local dynamic behavior of strange attractors, Phys. Rev. A, 45 (1992), pp. 7073-7084.

[3] L. CAO, Practical method for determining the minimum embedding dimension of a scalar time series, Phys. D, 110 (1997), pp. 43-50.

[4] M. Casdagli, S. Eubank, J. D. Farmer, and J. Gibson, State space reconstruction in the presence of noise, Phys. D, 51 (1991), pp. 52-98.

[5] A. M. Fraser and H. L. Swinney, Independent coordinates for strange attractors from mutual information, Phys. Rev. A, 33 (1986), pp. 1134-1140.

[6] J. F. Gibson, J. D. FARmer, M. CASDAGLi, And S. Eubank, An analytic approach to practical state space reconstruction, Phys. D, 57 (1992), pp. 1-30.

[7] C. W. J. Granger, Investigating causal relations by econometric models and cross-spectral methods, Econometrica, 1969, pp. 424-438.

[8] M. W. Hirsch, Differential Topology, Grad. Texts in Math. 33, Springer-Verlag, New York, 1976.

[9] J. P. Huke, Embedding Nonlinear Dynamical Systems: A Guide to Takens' Theorem, MIMS EPrint, 2006.

[10] J. P. Huke And D. S. Broomhead, Embedding theorems for non-uniformly sampled dynamical systems, Nonlinearity, 20 (2007), pp. 2205-2244.

[11] D. Kugiumtzis, State space reconstruction parameters in the analysis of chaotic time series - the role of the time window length, Phys. D, 95 (1996), pp. 13-28.

[12] W. Liebert, K. Pawelzik, and H. G. Schuster, Optimal embeddings of chaotic attractors from topological considerations, Europhys. Lett. EPL, 14 (1991), pp. 521-526.

[13] J. R. Munkres, Topology, Vol. 2, Prentice-Hall, Upper Saddle River, NJ, 2000.

[14] J. Palis and W. De Melo, Geometric Theory of Dynamical Systems, Springer, New York, 1982.

[15] L. M. Pecora, T. L. Carroll, and J. F. Heagy, Statistics for mathematical properties of maps between time series embeddings, Phys. Rev. E, 52 (1995), pp. 3420-3439.

[16] T. Sauer, J. A. Yorke, And M. Casdagli, Embedology, J. Stat. Phys., 65 (1991), pp. 579-616.

Copyright ( ) by SIAM. Unauthorized reproduction of this article is prohibited. 
[17] M. Stern, Semimodular Lattices: Theory and Applications, Cambridge University Press, Cambridge, UK, 1999.

[18] S. Strogatz, Nonlinear Dynamics and Chaos: With Applications to Physics, Biology, Chemistry and Engineering, Perseus Books Group, 1994.

[19] G. Sugihara, R. May, H. Ye, C. Hsieh, E. Deyle, M. Fogarty, and S. Munch, Detecting causality in complex ecosystems, Science, 338 (2012), pp. 496-500.

[20] F. Takens, Detecting strange attractors in turbulence, in Symposium on Dynamical Systems and Turbulence, D. A. Rand and L. S. Young, eds., Springer-Verlag, Berlin, 1981, pp. 366-381.

Copyright $\odot$ by SIAM. Unauthorized reproduction of this article is prohibited. 